\title{
High power impulse magnetron sputtering discharge
}

\author{
J. T. Gudmundsson, N. Brenning, Daniel Lundin and Ulf Helmersson
}

\section{Linköping University Post Print}

N.B.: When citing this work, cite the original article.

Original Publication:

J. T. Gudmundsson, N. Brenning, Daniel Lundin and Ulf Helmersson, High power impulse magnetron sputtering discharge, 2012, Journal of Vacuum Science \& Technology. A. Vacuum, Surfaces, and Films, (30), 030801.

http://dx.doi.org/10.1116/1.3691832

Copyright: American Vacuum Society, This article may be downloaded for personal use only. Any other use requires prior permission of the author and the American Vacuum Society.

http://www.avs.org/

Postprint available at: Linköping University Electronic Press

http://urn.kb.se/resolve?urn=urn:nbn:se:liu:diva-76304 


\title{
High power impulse magnetron sputtering discharge
}

\author{
J. T. Gudmundsson \\ University of Michigan-Shanghai Jiao Tong University Joint Institute, Shanghai Jiao Tong University, \\ 800 Dong Chuan Road, Shanghai 200240, China and Science Institute, University of Iceland, Dunhaga 3, \\ IS-107 Reykjavik, Iceland
}

N. Brenning

Division of Space and Plasma Physics, School of Electrical Engineering, Royal Institute of Technology, SE-100 44, Stockholm, Sweden

D. Lundin and U. Helmersson ${ }^{\text {a) }}$

Plasma \& Coatings Division, IFM-Materials Physics, Linköping University, SE-581 83, Linköping, Sweden

(Received 9 February 2012; accepted 12 February 2012; published 14 March 2012)

\begin{abstract}
The high power impulse magnetron sputtering (HiPIMS) discharge is a recent addition to plasma based sputtering technology. In HiPIMS, high power is applied to the magnetron target in unipolar pulses at low duty cycle and low repetition frequency while keeping the average power about 2 orders of magnitude lower than the peak power. This results in a high plasma density, and high ionization fraction of the sputtered vapor, which allows better control of the film growth by controlling the energy and direction of the deposition species. This is a significant advantage over conventional dc magnetron sputtering where the sputtered vapor consists mainly of neutral species. The HiPIMS discharge is now an established ionized physical vapor deposition technique, which is easily scalable and has been successfully introduced into various industrial applications. The authors give an overview of the development of the HiPIMS discharge, and the underlying mechanisms that dictate the discharge properties. First, an introduction to the magnetron sputtering discharge and its various configurations and modifications is given. Then the development and properties of the high power pulsed power supply are discussed, followed by an overview of the measured plasma parameters in the HiPIMS discharge, the electron energy and density, the ion energy, ion flux and plasma composition, and a discussion on the deposition rate. Finally, some of the models that have been developed to gain understanding of the discharge processes are reviewed, including the phenomenological material pathway model, and the ionization region model. (C) 2012 American Vacuum Society. [http://dx.doi.org/10.1116/1.3691832]
\end{abstract}

\section{INTRODUCTION}

Plasma based physical vapor deposition (PVD) has found widespread use in various industrial applications, in particular in coating processes. In plasma based PVD processes the deposition species are either vaporized by thermal evaporation or by sputtering from a source (the cathode target) by ion bombardment. Sputtering has been known for decades as a flexible, reliable, and effective coating method. The magnetron sputtering technique that was developed during the $1960 \mathrm{~s}$ and $1970 \mathrm{~s}$ has been the workhorse of plasma based sputtering applications for the past three decades. ${ }^{1-3}$ In the planar configuration the magnetron sputtering discharge is simply a diode sputtering arrangement with the addition of magnets directly behind the cathode. With the introduction of magnetron sputtering the disadvantages of diode sputtering, such as poor deposition rate, were overcome as the operating pressure could be reduced, ${ }^{1}$ while maintaining the energy of the sputtered species, ${ }^{1,4}$ often resulting in

${ }^{\text {a)} E l e c t r o n i c ~ m a i l: ~ u l f h e @ i f m . l i u . s e ~}$ improved film properties. ${ }^{4-6}$ Through the years there has been a continuous development of magnetron sputtering processes to increase metal ionization, improve target utilization, avoid target poisoning in reactive sputtering, increase deposition rates, and minimize electrical instabilities such as arcs.

For many applications a high degree of ionization of the sputtered vapor is desired as the ion flux to the substrate is known to have a significant influence on the overall quality of the resulting film. ${ }^{7,8}$ Ionized sputtered flux may be used to bring about controlled ion bombardment of the growing film by acceleration of the sputtered vapor across the plasma sheath created by a negative bias applied to the substrate. Ionized flux of the sputtered vapor therefore introduces an additional control parameter into the deposition process. It is referred to as ionized physical vapor deposition (IPVD) when the sputtered vapor has more ions than neutrals. ${ }^{9,10}$ Over the past decade various ionized sputtering techniques have appeared that can achieve a high degree of ionization of the sputtered vapor. ${ }^{11}$ Common to all the IPVD techniques is a very high density plasma. The development of 
IPVD techniques was initially mainly driven by the need to deposit metal layers and diffusion barriers into trenches or vias of high aspect ratio for integrated circuits, ${ }^{9,10,12-14}$ but has during the past years found a number of additional areas where the beneficial properties of increased ionization of the sputtered vapor can be utilized, which will be addressed in more detail at the end of this section. One of the most important process parameters is the plasma density, since it affects both the deposition rate as well as the ionization fraction of the sputtered material flux. The plasma density increases with increased power density supplied to the target. But increased power density leads to overheating and eventually melting of the sputtering target. Thus, there is an upper limit to the power that can be delivered through the discharge target. Initially magnetron sputtering based IPVD processes relied on a secondary discharge to create a dense plasma, placed between the source (the cathode target) and the substrate, to ionize a large fraction of the sputtered atoms. ${ }^{11,15}$ Recently, IPVD has been achieved by applying a high power unipolar pulse of low frequency and low duty cycle to the cathode to create a very high plasma density. ${ }^{11,16,17}$ This is referred to as high power pulsed magnetron sputtering (HPPMS). The HPPMS techniques have the advantage of using essentially the conventional magnetron sputtering equipment, except for the power supply. Thus, this technique can be implemented into full scale industrial size deposition systems, independent of the target geometry, with relative ease. There are a few variations of the HPPMS technique. In high power impulse magnetron sputtering (HiPIMS) a pulse of very high amplitude, an impulse, is applied to the cathode and a long pause exists between the pulses. In order to distinguish this technique from other pulsed magnetron processes Anders ${ }^{18}$ defines HiPIMS as pulsed magnetron sputtering, where the peak power exceeds the time-averaged power by typically 2 orders of magnitude. This is sometimes done by using a dc power supply to maintain a conventional dc magnetron sputtering (dcMS) discharge and then overlay high power pulses with low duty cycle, referred to as preionized HiPIMS. ${ }^{19}$ Other approaches include modulating the pulse such that in the initial stages of the pulse (few hundred microseconds) the power level is moderate (typical for a dcMS) followed by a high power pulse (lasting a few hundred microseconds up to a millisecond), and is referred to as modulated pulse power (MPP). ${ }^{20}$ The nomenclature for pulsed discharges used in this paper is based on the peak power density at the target $p_{\mathrm{t}}$, combined with the duty cycle (the percentage of the time that the pulse is on) as shown in Fig. 1. We take $p_{\mathrm{t}}=0.05 \mathrm{~kW} / \mathrm{cm}^{2}$ to be a typical upper limit for a dcMS before target damage sets in. Pulsed discharges below this limit are referred to as pulsed dcMS and include the bipolar asymmetrically pulsed discharges, operating in the medium frequency range $10-250 \mathrm{kHz}$, developed for reactive sputtering. ${ }^{21,22}$ All discharge operation above the dcMS limit is referred to as HPPMS. In this range, the higher peak power must be compensated for by a lower duty cycle. For square shaped pulses, this gives the power density limit line shown in Fig. 1. The HiPIMS range in peak power density is defined to lie above an HiPIMS limit,

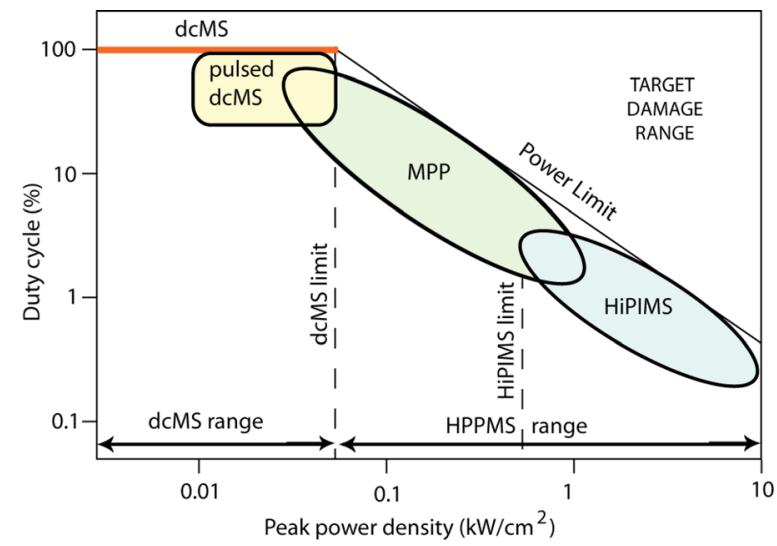

FIG. 1. (Color online) Nomenclature for pulsed discharges used in this paper is based on the peak power density at the target $p_{\mathrm{t}}$, combined with the duty cycle.

$p_{\mathrm{t}}>0.5 \mathrm{~kW} / \mathrm{cm}^{2}$. MPP pulses typically begin at a low power level, often in the dcMS range, followed by a stronger pulse of intermediate power density $\left(0.05<p_{\mathrm{t}}<0.5 \mathrm{~kW} / \mathrm{cm}^{2}\right)$ or even into the HiPIMS range. As development proceeds, a finer subdivision of the MPP discharges into various types is envisaged. An almost unexplored area is pulse train structuring, which we propose to mean cases when the time delays between the pulses are varied, rather than the pulse form.

The HiPIMS discharge operates with a cathode voltage in the range of $500-2000 \mathrm{~V}$, current densities of up to $3-4 \mathrm{~A} / \mathrm{cm}^{2}$, peak power densities in the range of $0.5-10 \mathrm{~kW} / \mathrm{cm}^{2}$, repetition frequency in the range of $50-5000 \mathrm{~Hz}$, and duty cycle in the range of $0.5 \%-5 \% .^{11,15}$ Although the peak power is $2-3$ orders of magnitude larger than commonly used in a conventional dcMS discharge, the average power is the same. The parameter range is much wider in the pulsed system compared to conventional dc and radio frequency (rf) operation and the pulsing of the discharge allows for a much greater flexibility due to additional control parameters such as pulse width, duty cycle, and pulse frequency. A thorough review on IPVD techniques and their applications was given by Helmersson et al. ${ }^{11}$ An early review focusing only on the HiPIMS discharge was given by Helmersson $e \mathrm{al}^{23}$ and a more thorough review was given by Sarakinos et al. ${ }^{24}$ More recently Anders ${ }^{18}$ gave a tutoriallike overview of the HiPIMS technique. A recent review of the MPP technique was given by Lin et $a .^{25}$ An introductory review on thin film processing using HiPIMS has been written by Lundin and Sarakinos ${ }^{26}$ and a brief review on the advantages of the HiPIMS technique for metallic thin film coatings was given by Alami et al. ${ }^{27}$

High ionization of the sputtered vapor leads to better control of the film growth through the application of substrate bias that can be used to control the ion arrival energy and guide the deposition material. It is well known that low energy working gas ion bombardment during deposition of thin films has a significant influence on the structure and properties of the growing film, including degree and direction of orientation, grain size, the film density, and film stress. ${ }^{7,8,28}$ Increased ionization of the sputtered vapor also gives improvement of the film quality, such as density, ${ }^{29-31}$ 
higher hardness, ${ }^{32,33}$ improved adhesion, ${ }^{34}$ improved surface roughness, ${ }^{35,36}$ control of the reactivity, the deposition temperature can be lower while maintaining the film quality, ${ }^{37}$ phase tailoring, ${ }^{38}$ and guiding of the deposition material to the desired areas of the substrate. ${ }^{39}$ Furthermore, due to the pulsed nature of the deposition flux the instant deposition rates are very high, giving a high supersaturation of adatoms on the growth surface. This leads to changed growth conditions with flat films and dense microstructures as a result. ${ }^{40}$ It has been shown that a higher deposition rate is achieved in HiPIMS on surfaces that are not parallel to the target surface, compared to deposition in a dcMS and that the film properties are equivalent to the films grown parallel to the target. ${ }^{41}$ Also, hysteresis-free reactive sputtering has been demonstrated in an HiPIMS discharge. ${ }^{42}$ All of these advantages are of particular importance for deposition on substrates of complex nature. ${ }^{27,41}$

Here the development of the HiPIMS discharge will be reviewed, its basic properties summarized, and the principle of operation explained by simple models. This review focuses on the discharge and its properties and does not discuss the nucleation and/or growth of thin films. In Sec. II the magnetron sputtering discharge is discussed and its various configurations in use today. This section is intended to be an introduction to magnetron sputtering for the nonexpert and also to emphasize that with the HPPMS techniques, IPVD can be utilized in various configurations. Then we discuss the development and properties of the high power pulsed power supply. But the pulsed power supply is what distinguishes the conventional dcMS from an HPPMS system. Section III gives an overview of the properties of the HiPIMS discharge and summarizes the experimental findings, such as the spatial and temporal variations of the plasma parameters and the energy of the electrons and ions. Keep in mind that the experimental characterization has been performed on various configurations and multiple combinations of cathode targets and gas mixtures. Also, some of these experimental results remain to be fully understood. Thus, this section is supposed to serve as a starting point for researchers entering the field. Section IV discusses the physics of the HiPIMS discharge and summarizes the models that have been constructed and simulations that have been performed to describe its properties and understand its behavior. This discussion starts with a simple phenomenological model and moves on to more involved discussion on transport properties of charged particles in the presence of electric and magnetic fields.

\section{MAGNETRON SPUTTERING}

The introduction of the magnetron sputtering discharge ${ }^{1}$ and its development into the planar magnetron sputtering discharge ${ }^{2}$ defined the advent of a new era in vacuum coating technology. Here we discuss briefly the development of the magnetron sputtering technique, the basic physics of its operation, and the modifications that have been made over the years. We describe the various configurations of the magnetron sputtering discharge that are in use both under labora- tory and industrial conditions. Then we move on to discuss the development, design, and characteristics of the pulsed power supply that is used to drive the HPPMS discharges, including HiPIMS and MPP.

\section{A. Planar magnetron sputtering discharge}

Sputtering is the ejection of atoms due to bombardment of a solid or a liquid surface (the target) by energetic particles, often ions. Secondary electrons are also emitted from the target surface as a result of the ion bombardment. The source of ions for the sputtering process is most easily achieved by a plasma discharge. Sputtering in gas discharges was discovered in the mid-19th century. ${ }^{43}$ But it gained significant interest in the late $1950 \mathrm{~s}$ and early $1960 \mathrm{~s}$ with improved vacuum technology and the realization that a wide range of materials could be deposited using sputtering, ${ }^{44,45}$ including dielectrics. ${ }^{46}$ These were often low aspect ratio $(L / R<1)$ dc glow discharges, the cathode serves as the target for ion impact sputtering, and almost all the applied voltage appears across the cathode sheath. Here $R$ is the discharge radius and the discharge length $L$ is typically a few centimeters, the negative glow extends almost to the anode, and the positive column is absent. For decades these discharges were used as a sputtering source for both metallic (dc glow discharge) and dielectric targets ( $\mathrm{rf}$ glow discharge). ${ }^{44-48}$ The discharge is maintained by the secondary electrons created at the cathode (the target) by the impact of energetic ions. These electrons sustain the discharge by accelerating from the cathode and ionizing the neutral working gas, which then bombards the cathode target and releases more secondary electrons. To achieve high enough ion current for commercially acceptable deposition rates very high discharge voltages have to be applied, of the order of $2-5 \mathrm{kV} .^{49}$ Since the discharge is maintained by secondary electron emission, the operating pressure must be high enough so that the secondary electrons are not lost to the anode or to the grounded surfaces before performing ionization. These pressures are higher than preferred for optimum transport of the sputtered deposition atoms due to scattering by the gas atoms. Hence, there is a narrow pressure range around $4 \mathrm{~Pa}$ for dc glow discharge sputtering to be viable. ${ }^{49}$ In order to lower the discharge voltage and expand the operation pressure range the lifetime of the electrons in the target vicinity had to increase. Penning ${ }^{50}$ had proposed the use of magnetic field in a sputtering system to extend the lifetime of the electrons escaping from the cathode, and trap them in the vicinity of the cathode target. Exploration of the effects of magnetic field on a dc glow discharge in diode configuration led to the discovery of an additional ionization region in the negative glow in the presence of magnetic field and increased ion intensity at the target. ${ }^{51}$ This idea was developed into a cylindrical hollow cathode device in which axial magnetic field is used to trap electrons, coined as magnetron sputtering. ${ }^{1}$ The enhanced ionization with magnetic field lead to acceptable deposition rates as well as operation at pressures significantly lower than $4 \mathrm{~Pa}^{1}{ }^{1}$ These coaxial cylindrical magnetron sputtering sources ${ }^{52,53}$ were demonstrated 
in both hollow cathode ${ }^{1,6}$ and center cathode configurations. ${ }^{54,55}$ These were followed by the introduction of the planar magnetron sputtering device. ${ }^{2,3}$ In the planar configuration, the magnetic field can be created by permanent magnets, ${ }^{2}$ electromagnets, ${ }^{56,57}$ or a combination of both. ${ }^{58,59} \mathrm{~A}$ schematic of a dc planar magnetron sputtering configuration is shown in Fig. 2. In the planar configuration the magnetic field lines go out in the center of the cathode and go back into the cathode at the annular. If the cathode plate is circular (can also be rectangular), the magnetic confinement is seen as a torus shaped plasma that hovers in front of the target and leads to the formation of a circular grovelike erosion pattern (the "race track") on the target surface.

Sometimes a planar magnetron discharge consists of a planar cathode (sputtering source or target) parallel to an anode surface. However, in most cases the anode is the grounded shield around the magnetron target (as seen in Fig. 2). Since the electrons will move along magnetic field lines with ease, the magnetic field lines closest to the cathode target that go through a grounded structure, e.g., the ground shield, will define a virtual anode for the magnetron. The position of the anode, including the virtual anode, is very important for the interaction between the plasma and the substrate. If the anode shields the plasma generated at the cathode from the substrate, the plasma will be very weak in the substrate vicinity and the possibility to utilize the plasma to modify the growing film, with, e.g., low-energy ion bombardment, is very limited.

In a conventional dcMS discharge the cathode is kept at a constant negative voltage. Positive ions generated in the plasma are accelerated toward the cathode target generating a vapor of atoms and molecules from the target surface through sputtering. In the magnetron sputtering discharge the secondary electrons are accelerated by the potential difference between the cathode and the bulk plasma. The main advantage of the planar magnetron is that the sputtered material flows in the direction normal to the cathode plane and will uniformly coat a surface that is translated past the magnetron target. Conventional planar dcMS sources are commonly operated using argon as the working gas in the pressure range $0.1-1.5 \mathrm{~Pa}$ and the applied cathode voltage in the range of 300-700 V. This leads to current densities of the order of $4-60 \mathrm{~mA} / \mathrm{cm}^{2}$ and power densities of several tens of

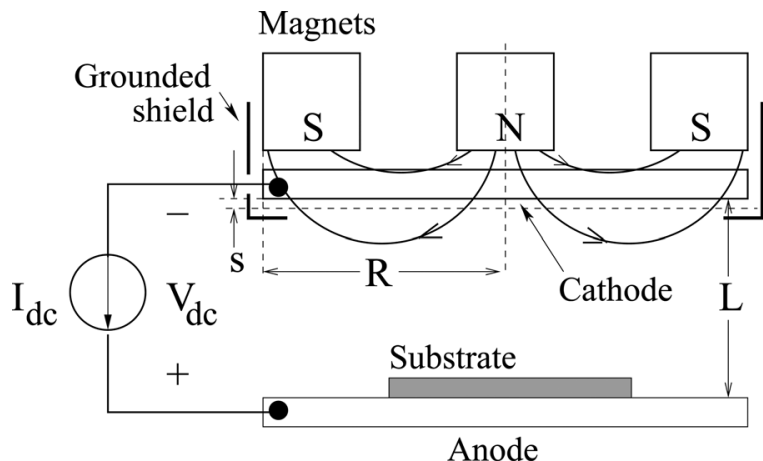

FIG. 2. Schematic of the dc planar magnetron discharge used for sputtering.
$\mathrm{W} / \mathrm{cm}^{2} .^{3}$ This pressure regime and operation parameters lead to a collisional-free sputter deposition process where the deposition rate is limited by the target power density and the sputtered atoms maintain their energy of a few eV obtained from the sputtering event. The electron density in the substrate vicinity is typically in the range $10^{15}-10^{17} \mathrm{~m}^{-3} \cdot{ }^{60-63}$ The static deposition rate is in the range $1-10 \mathrm{~nm} / \mathrm{s}$. The degree of ionization of the sputtered vapor is generally very low, often on the order of $0.1 \%$ or less. ${ }^{64}$ The majority of the ions bombarding the substrate are ions of the working gas. Also, the density of the sputtered particles is much lower than the density of the noble working gas. ${ }^{65}$ The primary mechanism for the ionization of the sputtered metal vapor in a dcMS is Penning ionization through impact with the working noble gas atoms that are in the metastable state. ${ }^{66}$ The mean free path for the sputtered vapor with respect to electron impact ionization is over $50 \mathrm{~cm}$. ${ }^{67}$

The cathode current voltage characteristics are found to follow the relationship

$$
I_{\mathrm{d}}=k V_{\mathrm{d}}^{n}
$$

where $I_{\mathrm{d}}$ is the discharge current (or cathode current), $V_{\mathrm{d}}$ the discharge voltage (or cathode voltage), and $n$ is in the range between 3 and 15. 3,53,68,69 Equation (1) is purely an empirical observation. The exponent $n$ depends on the efficiency of the electron trapping in the plasma. The more efficient the electron trapping is the higher the $n$ value. ${ }^{3} \mathrm{~A}$ high value of $n$ indicates that the discharge can accommodate a significant increase in discharge current with a relatively small change in target voltage. This can be achieved since the plasma is relatively weakly ionized and more charge carriers are generated as needed. ${ }^{70}$ The constant $k$ depends on the target material, gas pressure, gas type, magnetic field shape, and the geometry of the discharge. As the discharge is sustained by secondary electron emission from the cathode by ion bombardment, the discharge current at the target consists of electron current $I_{\mathrm{e}}$ and ion current $I_{\mathrm{i}}$ or

$$
I_{\mathrm{d}}=I_{\mathrm{e}}+I_{\mathrm{i}}=I_{\mathrm{i}}\left(1+\gamma_{\mathrm{SE}}\right),
$$

where $\gamma_{\mathrm{SE}}$ is the secondary electron emission coefficient. Note that $\gamma_{\mathrm{SE}} \sim 0.05-0.2$ for most metals, ${ }^{71}$ so at the target, the dominating fraction of the discharge current is ion current.

The number of electron-ion pairs created by each secondary electron that is trapped in the target vicinity is then

$$
\mathcal{N} \approx \frac{V_{\mathrm{d}}}{\mathcal{E}_{\mathrm{c}}}
$$

where $\mathcal{E}_{\mathrm{c}}$ is the energy loss per electron-ion pair created with the flow of secondary electrons into the plasma as the source of energy. ${ }^{49,52,72}$ But, not all the secondary electrons are confined in the target vicinity. To account for the electrons that are not trapped, Thornton and Penfold ${ }^{52}$ assume an effective secondary electron emission coefficient,

$$
\gamma_{\mathrm{SE}, \mathrm{eff}} \approx \frac{1}{2} \gamma_{\mathrm{SE}}
$$


that takes into account both the return probability and the loss of secondary electrons due to poor electron trapping. To sustain the discharge the condition,

$$
\gamma_{\mathrm{SE}, \mathrm{eff}} \mathcal{N}=1
$$

has to be fulfilled. This defines the minimum voltage to sustain the discharge as

$$
V_{\mathrm{d}, \min } \approx \frac{2 \mathcal{E}_{\mathrm{c}}}{\gamma_{\mathrm{SE}}} .
$$

Electrons in the target vicinity are magnetized, i.e., their gyration radius is much smaller than the characteristic size of the confining magnetic field structure. As seen in Fig. 2 the magnetic field is arched and the electrons are reflected back into the ionization region above the race track whenever they encounter the cathode sheath edge. They bounce back and forth along the magnetic field lines in cycloidallike trajectories until a collision occurs. The majority of the ionization events occur in this region where the energetic electrons are trapped. The path of the electrons is more complicated due to the presence of both a magnetic field $\mathbf{B}$ and an electric field $\mathbf{E}$. In the absence of an electric field an electron will gyrate in the magnetic field with the electron cyclotron angular frequency,

$$
\omega_{\mathrm{e}}=\frac{e B}{m_{\mathrm{e}}},
$$

and the corresponding gyration radius is

$$
r_{\mathrm{ce}}=\frac{u_{\mathrm{e}, \perp}}{\omega_{\mathrm{e}}}=\frac{u_{\mathrm{e}, \perp} m_{\mathrm{e}}}{e B},
$$

where $u_{\mathrm{e}, \perp}$ is the electron speed perpendicular to the magnetic field $\mathbf{B}$. In the presence of an electric field the electron exhibits a net drift perpendicular to both the $\mathbf{B}$ and $\mathbf{E}$ field vectors, often referred to as the Hall drift, or the $\mathbf{E} \times \mathbf{B}$ drift, ${ }^{49,73}$ which is given by

$$
v_{\mathrm{E}}=\frac{\mathbf{E} \times \mathbf{B}}{B^{2}},
$$

and the electrons perform trochoid movements. For a planar magnetron discharge this drift is in the azimuthal direction and the resulting azimuthal current is often referred to as the Hall current. ${ }^{53,74}$ In addition, there is a drift driven by the electron pressure gradient (or diamagnetic drift) written as

$$
v_{\nabla \mathrm{p}}=\frac{\nabla p_{\mathrm{e}} \times \mathbf{B}}{e n_{\mathrm{e}} B^{3}} .
$$

The Hall drift [Eq. (9)] and the diamagnetic drift [Eq. (10)] result in an azimuthal current flowing above the target race track. Notice that, as pointed out by, e.g., Thompson, ${ }^{75}$ the curved vacuum $\mathbf{B}$ field drifts (the drifts of the gyro centers that are proportional to the electron energies $\mathcal{E}_{\|}$and $\mathcal{E}_{\perp}$ ) give no contribution to the macroscopic current in a homogeneous plasma. Ions have a gyration radius $r_{\mathrm{ci}}$ that is larger than the characteristic size of the system, and thus the ions are not magnetized by the relatively weak static magnetic field.

\section{B. Modifications to the magnetron sputtering discharge}

Over the years various modifications have been made to improve the magnetron sputtering technology (see, e.g., the reviews by Kelly and Arnell ${ }^{76}$ and Sproul ${ }^{77}$ ), in particular to increase the flux of ions to the substrate and to allow deposition of dielectric films. The magnetron sputtering technique can be applied to a large variety of materials, and is easily scalable to large areas. The coating uniformity can be in the range of a few percent even for cathodes in the range of meters. Over the past decades magnetron sputtering has become an extremely important technology for thin film deposition in a wide range of industrial applications. These include metalization in integrated circuits, ${ }^{9,14}$ coatings for wear resistance and corrosion protection, ${ }^{78}$ large area coating of architectural glass, ${ }^{79}$ and display applications. ${ }^{80}$ Depending on the application the applied target voltage can be dc, radio frequency, ${ }^{81}$ or pulsed. ${ }^{82}$ The pulsed magnetron sputtering arrangement can be either an asymmetric bipolar pulsed $^{21,22,83}$ or an unipolar pulsed ${ }^{16}$ discharge. Conventional dcMS is ideal for depositing thin metallic films from electrically conducting targets. When depositing from thick electrically insulating (often compound) target materials, rf power is the only option. Compounds such as oxides and nitrides can also be deposited with reactive sputtering, in which a metal target is sputtered inside a discharge of reactive gas. The asymmetric bipolar dc sputtering discharge was developed to optimize the deposition of insulating films from conductive targets with reactive sputtering. ${ }^{21}$ Pulsing the magnetron discharge in the medium frequency range $(10-250 \mathrm{kHz})$ when depositing insulating films can significantly reduce the formation of arcs and; consequently, reduce the number of defects in the resulting film. In asymmetric bipolar mode the target is pulsed between the normal operating voltage and a slightly positive (roughly 10\%-20\% of the negative voltage amplitude) voltage for a short duration. More recently, high-power pulsed magnetrons in unipolar mode have been proposed for highly ionized sputtering. ${ }^{16,17}$ Over the past two decades there has been significant progress in enhancing the level of ionization in the magnetron sputtering discharge. This was initially achieved by the application of a secondary discharge to a conventional magnetron sputtering discharge, ${ }^{15}$ either an inductively coupled plasma source (ICP-MS) ${ }^{12,84-86}$ or a microwave amplified magnetron sputtering. ${ }^{87-90}$ The secondary discharge typically creates plasma with electron density in the range of $10^{17}-10^{18} \mathrm{~m}^{-3}$ and with electron temperature in the range of $1.5-4.5 \mathrm{~V},{ }^{91,92}$ which corresponds to electron impact ionization mean free path for the sputtered vapor of a few centimeters. ${ }^{9,67}$ The ICP-MS discharge is currently widely used in the semiconductor industry for deposition of metal and compound lines, pads, vias, and contacts. ${ }^{14}$ The combination of magnetron sputtering and secondary high density discharges was also applied to demonstrate a 
collisionless deposition process at pressures below $0.1 \mathrm{~Pa}^{89,93}$ The low pressure sputtering can be realized with improved plasma confinement or a secondary discharge that provides additional ionization of the working gas. ${ }^{93}$ HiPIMS is a sputtering technique where a high density plasma is created by applying high power pulses at low frequency and low duty cycle to a magnetron sputtering device. Then the peak electron density is in the range of $10^{18}-10^{19} \mathrm{~m}^{-3}, 94,95$ which corresponds to an electron impact ionization mean free path for the sputtered vapor of order of $1 \mathrm{~cm}$ or less. ${ }^{67}$ These discharges are the subject of this review.

The conventional planar magnetron sputtering discharge is considered to be balanced if the magnetic fluxes through the pole faces of the outer poles and through the pole face of the inner pole are similar. If the condition is fulfilled the magnetic trap confines the plasma just in front of the cathode target. The substrate thus experiences very little impingement by ions, which is useful when depositing on heat sensitive substrates. The unbalanced magnetron was developed as an attempt to increase the ion current density to the substrate. ${ }^{56,96,97}$ A strengthening or weakening of the magnetic flux through one of the poles leads to an unbalance in the magnetic circuit. Window and Savvides ${ }^{56}$ define two types of unbalancing. In type I, all field lines originate from the central magnet with some not passing into the annular magnet. So in this case the unbalanced field lines are directed toward the chamber walls, leading to low plasma density in the substrate vicinity. In type II, all the field lines originate from the annular magnet with some not passing into the central magnet. These unbalanced field lines extend into the substrate vicinity. Some of the secondary electrons can follow these magnetic field lines away from the target toward the substrate. Thus, the plasma is not strongly confined to the target region, but is allowed to flow out toward the substrate. This leads to a significant increase in the ion current density in the vicinity of the substrate. ${ }^{77,97}$ As a consequence the energy of the ions bombarding the substrate during film growth can be tuned by the substrate bias.

\section{Discharge configuration}

The planar magnetron sputtering discharge is well established for deposition of thin films of both metallic and dielectric materials. It is widely used in both laboratory settings (using small circular targets) and in industrial applications where the targets are linear (rectangular). For industrial applications the productivity depends on the deposition rate and the sputter target utilization. One disadvantage of the planar configuration is the nonuniform magnetic field on the target surface that leads to nonhomogeneous ion current distribution across the target surface and thus induces a localized erosion area on the target (the race track). This implies a low utilization of the target. For a planar magnetron sputtering discharge the target utilization is often in the range $26 \%-45 \%$. $^{2,3}$ However, this issue has been resolved to a large degree by using rotating magnetic assemblies that increase the target utilization and improve the film thickness homogeneity dramatically, ${ }^{98,99}$ and target utilization of up to
$77 \%$ has been reported using an asymmetric yoke magnet structure. ${ }^{100}$

The rotable cylindrical magnetron target was proposed to further alleviate the problem of low utilization. ${ }^{101}$ Then the cathode target is a cylindrical tube and the magnet assembly is installed inside the cylinder as seen in Fig. 3. The target can rotate during the sputtering so the target erodes uniformly as the target material is continuously exposed to the plasma zone, resulting in a uniform erosion around $360^{\circ}$ of the target surface. Thus the target life increases substantially and the target utilization is as high as $90 \%$. In the cylindrical configuration the target surface area may be in hundreds and up to tens of thousands of square centimeters. In some cases the magnet assembly is wobbled for improved layer thickness distribution. ${ }^{80}$ The rotating magnetron sputtering discharge is widely used for large-scale industrial applications, in particular in large in-line flat glass coating plants. ${ }^{79}$ For large area coatings, using a typical in-line coater, the substrate is moved relative to the magnetron cathode target in a linear fashion. In particular for reactive sputtering the active region of the rotatable target is maintained free of dielectric layer build up due to continuous sputtering with rapid rotation speeds $(20 \mathrm{rpm}){ }^{79}$

It is difficult to coat uniformly complex components at acceptable rates from a single source. Therefore, in order to exploit the magnetron sputtering technology to large scale, industrial systems consisting of multiple magnetron sputtering targets have been developed. ${ }^{76}$ A dual magnetron sputtering system can be set up in either closed field or open field (mirror) configuration. ${ }^{102}$ In the closed field configuration the two magnets have opposite polarity and in the open field configuration they have the same polarity. So, in the closed field configuration, the field lines are linked between the magnetrons, losses to the chamber walls are low, and the substrate is situated in a high density plasma (note that this arrangement requires unbalanced magnetrons of type II). The dual configuration is often applied to enhance process stability in large industrial coaters where the power is switched between two cathodes of the same material. The two magnetrons alternately act as a cathode and anode, so a coating of the anode by an insulating layer during reactive growth, phenomena referred to

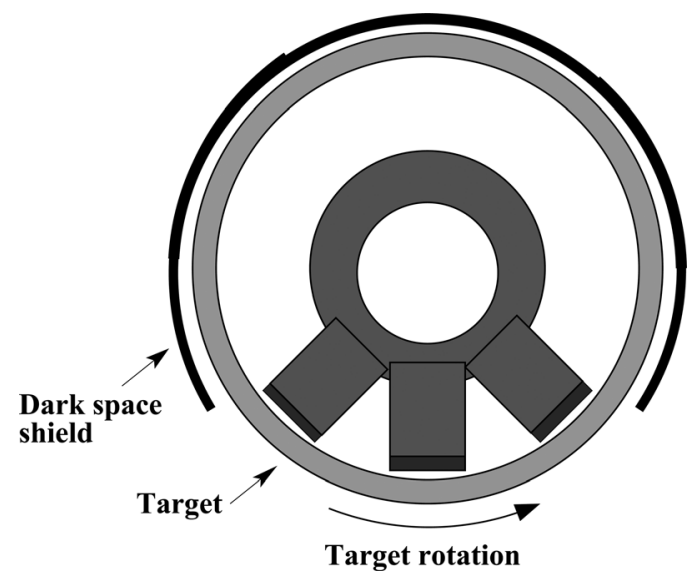

FIG. 3. Rotating magnetron sputtering discharge. The cathode target is a tube that rotates around the fixed magnet assembly with a frequency of roughly $1 \mathrm{~Hz}$. After Wright and Beardow (Ref. 101). 
as the disappearing anode effect, can be avoided. Hence, the dual configuration can provide a stable operation over a long process time and thus the dual magnetron sputtering systems are the preferred choice for large area coating on an industrial scale. ${ }^{103}$ It is also frequently applied for the growth of complex materials of varying stoichiometry by varying the power to the targets independently as well as the target to substrate distance. ${ }^{104} \mathrm{~A}$ dual magnetron sputtering system for deposition inside tubular substrates has been developed using a combination of dcMS and HiPIMS discharges arranged in the open field configuration ${ }^{105}$ and a dual HiPIMS discharge in the closed field configuration has been demonstrated. ${ }^{106}$

In the closed configuration (using unbalanced magnetrons of type II, with stronger outer poles) a virtual anode between the magnetrons can be avoided, and the expansion of the plasma to fill the space between the magnetrons is promoted. This was developed further into unbalanced multimagnetron sputtering systems referred to as closed field unbalanced multimagnetron systems (CFUBMS). ${ }^{107}$ In the CFUBMS configuration, magnetrons with alternating polarities, and hence joining of magnetic field lines between magnetrons, are lined up using even numbers of magnetrons $(2,4,6,8)$, and surround a rotating substrate holder. As demonstrated in Fig. 4 these adjacent magnetrons have opposite magnetic polarities and the field lines are closed. This leads to significantly enhanced ionization in the substrate vicinity. This configuration has been used successfully in industrial scale systems for almost two decades.

\section{Pulse power supply}

As discussed in Sec. II A, for a conventional dcMS discharge the power loading is typically a few tens $\mathrm{W} / \mathrm{cm}^{2}$. For

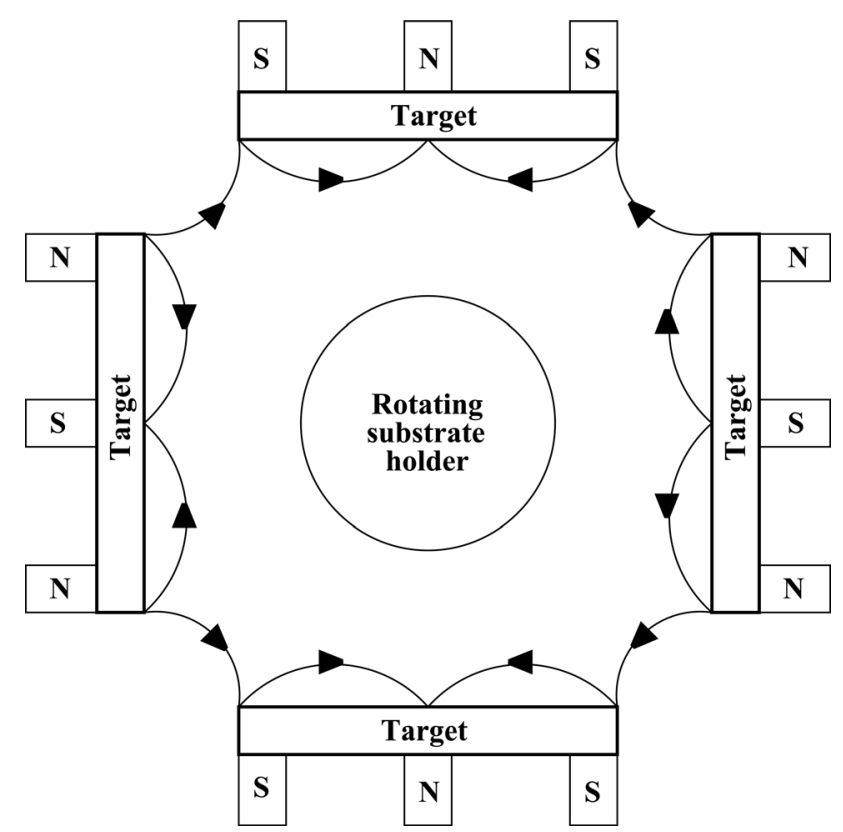

FIG. 4. Top view of a closed field unbalanced multimagnetron system commonly used in industrial scale configurations. Here four magnetrons with alternating polarities are lined to surround a rotating substrate holder. After Kelly et al. (Ref. 78). the HiPIMS discharge the power loading during the pulse can peak to several $\mathrm{kW} / \mathrm{cm}^{2}$. It is this high power density that is the key to achieving the dense plasma and the high degree of ionization of the sputtered vapor. At first glance the only difference between a conventional dcMS system and an HiPIMS system is the pulsed power supply. However, in reality some modification of the magnetron may also be necessary, which may include increased isolation to handle higher potentials, weakening the magnetic field to avoid too strong deposition rate loss, and improved cooling. Various pulsed power supplies have been developed to drive the HiPIMS discharge. Currently there are several commercially available HiPIMS power supplies capable of driving large area sputter targets. Here we first review the historical development of the pulsed power supply and its introduction to the magnetron sputtering technology. Then we describe the basic circuit and the circuit parameters that are used to create the high power pulse. Finally, we discuss the temporal variation of the discharge current and voltage, and their features.

\section{Historical remarks}

The design of the pulse generators is based on the technology developed to drive radars in the $1940 \mathrm{~s},{ }^{108}$ and xenon flash-lamp pump sources for solid state lasers in the 1960 s. ${ }^{109}$ They have more recently been developed to drive pulsed arc deposition sources. ${ }^{110,111}$ The historical development of the power supplies for the HiPIMS discharge has recently been reviewed by Ochs et al. ${ }^{112}$ They trace their development to the Moscow Engineering and Physics Institute in the early $1970 \mathrm{~s}$ where the initial work was on glow discharges (without magnetic confinement). In 1993 Mozgrin et al. ${ }^{113}$ reported a peak power of $200 \mathrm{~kW}(200 \mathrm{~A})$ onto a $1200 \mathrm{~mm}$ diameter target giving $1.8 \mathrm{~kW} / \mathrm{cm}^{2}$ at a repetition rate of $10 \mathrm{~Hz}$. Bugaev et al. ${ }^{114}$ report on a pulsed power supply that operates at repetition frequency $5-20 \mathrm{~Hz}$ and pulse duration $2-10 \mathrm{~ms}$, and gives peak power of $4 \mathrm{~kW}$, for pulsing a filament-assisted hollow cathode magnetron with a pulse voltage up to $800 \mathrm{~V}$ and a peak current of $450 \mathrm{~A}$. They report cathode current densities as high as $2.8 \mathrm{~A} / \mathrm{cm}^{2}$. Kouznetsov et al. ${ }^{16}$ discuss a thyristor based power supply capable of peak power pulses of up to $2.4 \mathrm{MW}$ at repetition frequency $50 \mathrm{~Hz}$ and pulse width $50-100 \mu$ s generating a peak target power density as large as $2.8 \mathrm{~kW} / \mathrm{cm}^{2}$ on a planar magnetron target. Christie et al. ${ }^{115}$ report on a power supply capable of peak powers of up to $3 \mathrm{MW}$ operating at single shot to $500 \mathrm{~Hz}$ repetition frequency and pulse width of $100-150 \mu \mathrm{s}$. These early HiPIMS power supplies are characterized by an initial voltage peak in the kilovolt range followed by a drop in the voltage to several hundred volts, typical of the operating voltage of a conventional dcMS. After a slight delay from the inital voltage peak the target current increases up to a peak value which also decays with falling voltage (see the discussion by Gudmundsson et al. ${ }^{95}$ ). A power supply that provides a constant voltage (a nearly rectangular waveform) throughout the length of a $400 \mu$ s long pulse due to a large storage capacitance has also been developed for HiPIMS applications (SPIK2000 A) ${ }^{116}$ (see, e.g., Anders et al. ${ }^{117}$ ). 
Musil et al. ${ }^{118}$ report on experiments with a pulsed power supply (Rübig MP120) that works in the frequency range $0.5-50 \mathrm{kHz}$ with maximum peak voltage of $1000 \mathrm{~V}$ and a peak current of $120 \mathrm{~A}$, and thus maximum pulse power of $120 \mathrm{~kW}$. Another approach to HPPMS is arbitrary tailored pulse shape utilizing multistep pulses, as introduced by Chistyakov et al., ${ }^{119,120}$ and referred to as MPP (see Fig. 1). Typically, a stable weakly ionized discharge is formed with a low power density (typical for dcMS) prior to a transition to a strongly ionized discharge created with a power density of $0.1-1.5 \mathrm{~kW} / \mathrm{cm}^{2}$ with a pulse length up to $3 \mathrm{~ms}$ and pulse frequency in the range $4-400 \mathrm{~Hz}^{25}$ Ehiasarian and Bugyi ${ }^{121}$ demonstrate a pulse generator (HMP 6/16) that supplies up to $3000 \mathrm{~A}$ at $2000 \mathrm{~V}$ in $0-200 \mu$ s pulses at $0-100 \mathrm{~Hz}$ frequency for large area cathodes $\left(>400 \mathrm{~cm}^{2}\right)$. For industrial applications the HiPIMS technique was demonstrated on linear magnetron targets of $400 \mathrm{~cm}^{2}$ area ${ }^{121}$ and later of $1200 \mathrm{~cm}^{2}$ area. ${ }^{122,123}$ Leroy et al. ${ }^{124}$ have demonstrated HiPIMS using a rotating cylindrical magnetron. They operated the discharge up to power densities of $2.7 \mathrm{~kW} / \mathrm{cm}^{2}$. It should be noted however that for applications in glass and web coating the cylindrical target area can be as large as a several times $101000 \mathrm{~cm}^{2}$, and thus the peak power has to be in tens of MW. Currently, a power supply that operates at voltages up to $3 \mathrm{kV}$ and can give a peak current of $6 \mathrm{kA}$ and thus a pulse power of $18 \mathrm{MW}$ is in production. ${ }^{125}$

\section{Pulse generator}

Pulser technology is often based on a switched capacitor bank in which a thyristor (historically a vacuum tube) switch is used to connect an energy storage capacitor to the load to initiate the pulse and then disconnect it at the end of the pulse. Sometimes this is arranged into what is referred to as line type pulsers. The energy storage device is essentially a lumped-constant transmission line $\mathrm{e}^{108}$ and consists of a lumped element network approximating a transmission line. ${ }^{126}$ This component of the line-type pulser is both the source of the electrical energy during the pulse as well as the pulse-shaping element and is generally referred to as the pulse-forming network. The design of a power supply for a high power pulsed magnetron sputtering is discussed by Christie et al., ${ }^{115,127}$ Sproul et al., ${ }^{128}$ and Kouznetsov. ${ }^{129}$ It is usually based on the pulse-forming network and consists of a single or multiple mesh $L C$ network as seen in Fig. 5. These systems operate in a repetitively pulsed manner. The pulse generator consists of a discharge capacitor which is connected to be charged from a charging circuit through a thyristor switch. The energy for the pulse is stored in an electrostatic field in the amount $(1 / 2) C_{\mathrm{s}} V_{0}^{2}$, were $C_{\mathrm{s}}$ is the network storage capacitance and $V_{0}$ is the peak charge voltage on the capacitor. The charging circuit generally consisted of a power supply and a charging element. The use of an inductance makes it possible to design a very highly efficient resonant charging circuit. ${ }^{130}$ Resonance charging of the capacitor is most often accomplished from a dc voltage source through a thyristor switch. ${ }^{113,131}$ The power supply can also be a transformer that has its primary winding connected to line mains and its secondary winding connected to the discharge capacitor through a diode and a thyristor switch. ${ }^{129}$ The pulse forming line is switched to the magnetron discharge through another thyristor. The pulse shaping element can be either a resistance or an inductance. If the pulse shaping element is an inductance coil it is connected in series with the magnetron discharge for pulse shaping (matching) in order to reduce the rate of current rise. This inductor often has a variable inductance in order to match various cathodes and to eliminate oscillations. ${ }^{125}$ This inductor is chosen so that it limits the current peak of the discharge in order to obtain the desired plasma parameters of the sputtering gas. ${ }^{115,129}$ Without the inductor connected in the circuit the current during the discharge of the capacitor the current could rise to hundreds of kiloamperes. The size of the discharge capacitor is typically of the order of $1-50 \mu \mathrm{F}$ and the inductor has an inductance of the order of $20-100 \mu \mathrm{H} .{ }^{59,115,127,129}$ The charge voltage $V_{0}$ varies from hundreds of volts to several thousand volts. The pulse repetition rate is generally varied from a few $\mathrm{Hz}$ to $5000 \mathrm{~Hz}$.

Some of the high power pulse systems include a preionizer. ${ }^{19,113,131-134}$ An electric circuit diagram illustrating a pulse generator with a preionizer is shown in Fig. 6 . Here a dc power supply maintains a conventional dcMS discharge. The storage capacitor $C_{\mathrm{s}}$ is charged through a thyristor switch from a charging circuit and a trigger circuit discharges the capacitor through a thyristor switch.

Christie et al. ${ }^{115}$ and Sproul et al. ${ }^{128}$ point out that arc handling is required for practical application of HiPIMS. Arc can be detected in various ways. ${ }^{115}$ One way is to monitor the discharge current: when the current increases beyond a set threshold an arc is detected. Also, the time derivative of the current can be monitored in order to detect any fast current rise characteristic for arcs. Similarly a sudden voltage decrease can be used to detect an arc. When arc suppression initiates, the increase in current is stopped by disconnecting the capacitor from the inductor and then disconnecting the inductor from the discharge load. At last the inductor energy is recycled into the capacitor.

\section{Discharge current and voltage waveforms}

To describe the discharge current-voltage characteristics the current-voltage-time space is required. The exact shapes of the current and voltage waveforms are to a great extent determined by the size of the storage capacitor $C_{\mathrm{s}}$, and also depend on whether the circuit inductance $L$ or the resistance of the plasma discharge dominate in limiting the current. When the inductance $L$ is relatively small the output voltage rises rapidly to a sharp peak. Then the output voltage

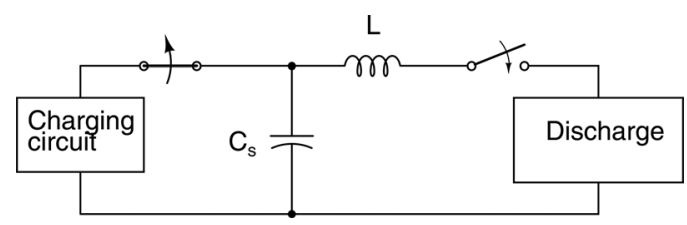

FIG. 5. Basic single mesh $L C$ network between the charging power supply and the discharge load. 


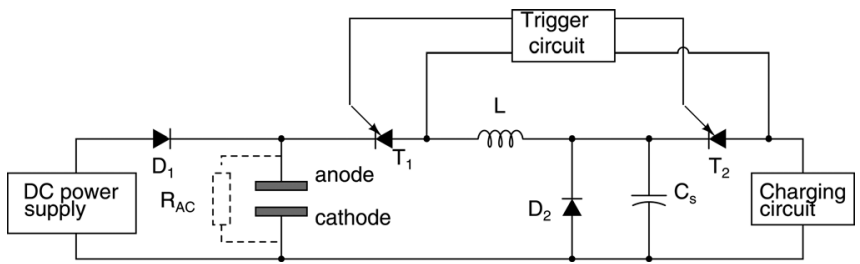

FIG. 6. Electric circuit diagram illustrating a pulse generator with a preionizer. A dc power supply maintains a conventional dc magnetron discharge. The storage capacitor $C_{\mathrm{s}}$ is charged through a thyristor switch $\left(\mathrm{T}_{2}\right)$ from a charging circuit and a trigger circuit discharges the capacitor through a thyristor switch $\left(\mathrm{T}_{1}\right)$.

decreases to a level that is determined by the discharge properties. This output voltage slowly decreases as the capacitor discharges, until it has dropped to a level where it cannot sustain the plasma. As the plasma dies, the current decreases to a low level. When the inductance $L$ is so large that it, and not the plasma resistance, is limiting the current, the pulse width can be estimated as ${ }^{115}$

$$
t_{\mathrm{w}} \approx 3 \sqrt{L C_{\mathrm{s}}}
$$

and is typically in the range $50-500 \mu \mathrm{s}$. The exact pulse shape is also determined by the load, the discharge formed in the sputtering device, and depends thus on the gas type, gas pressure, and magnetron design and size. An example of the temporal variations of the cathode voltage and discharge current can be seen in Fig. 7 measured using the same power supply (SINEX I) as used in the seminal work of Kouznetsov et al. ${ }^{16}$ It can be seen that the waveform of the cathode voltage and the discharge current depend on the discharge gas
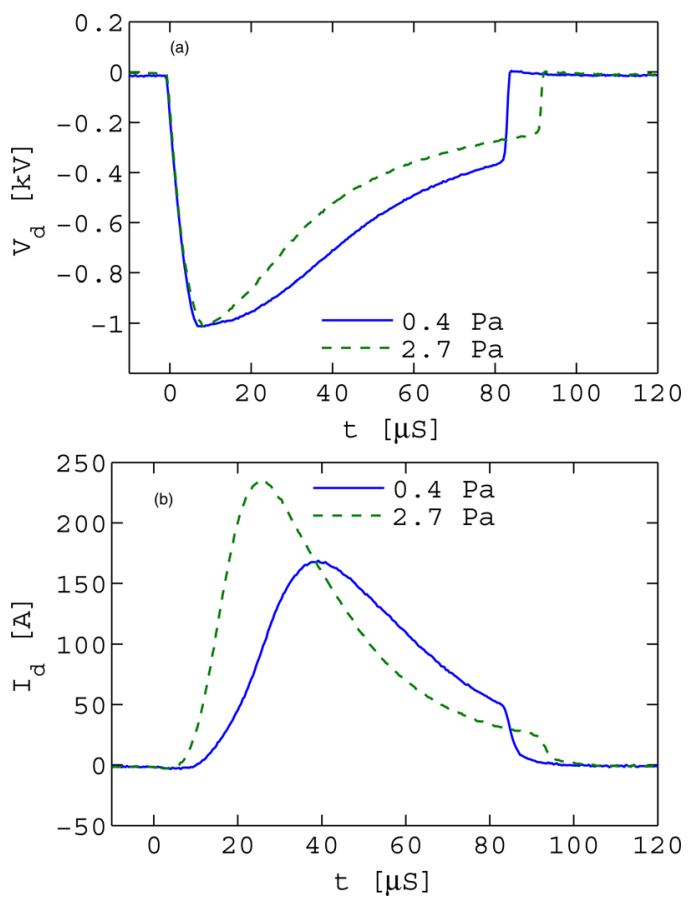

FIG. 7. (Color online) (a) Applied target voltage $V_{\mathrm{d}}$ and (b) the applied target current $I_{\mathrm{d}}$ for an argon discharge at 0.4 and $2.7 \mathrm{~Pa}$. The target is made of copper $150 \mathrm{~mm}$ in diameter. After Gudmundsson et al. (Ref. 135). pressure. The actual pulse width decreases with increasing discharge gas pressure. In the initial phase the applied voltage is in the kilovolt range. The voltage then drops to several hundred volts, which are typical operating voltages for a dcMS discharge. As the voltage drops the discharge current increases up to a peak value followed by a decay of the current. Generally, the discharge does not reach stationary plasma discharge conditions for pulse lengths of $100 \mu$ s or less. Some pulser units have a storage capacitor $C_{\mathrm{s}}$ that is large enough to keep a constant voltage for a relatively long pulse. Using a pulse power supply that keeps a constant voltage throughout the pulse length of $400 \mu \mathrm{s}$, Anders et al. ${ }^{117}$ recorded a set of current waveforms each taken at a fixed voltage for various target materials. They demonstrated that the current waveform for the HiPIMS discharge typically exhibits an initial pressure dependent peak that is followed by a second phase that is power and target material dependent. They claim that the initial peak is dominated by the ions of the sputtering gas while the later phase has a strong contribution from self-sputtering. To reach the latter phase the pulse length has to be longer than 100-200 $\mu$ s depending on the target material.

A delay is always noticed between the target voltage and the discharge current. This can be clearly seen in Fig. 7 and this delay time decreases as the pressure is increased from 0.4 to $2.7 \mathrm{~Pa}$. This delay depends on the gas pressure, ${ }^{95}$ gas composition, ${ }^{136}$ target material, ${ }^{137}$ and applied voltage. ${ }^{138}$ The delay time increases with decreasing pressure and can be in the range of a few $\mu$ s to over $100 \mu \mathrm{s}{ }^{95,134}$ Yushkov and Anders ${ }^{138}$ explore this phenomena both experimentally and theoretically. The delay of current onset consists of a statistical time lag $t_{\mathrm{s}}$ and the formative time lag $t_{\mathrm{f}}$ or

$$
t_{\mathrm{d}}=t_{\mathrm{s}}+t_{\mathrm{f}}
$$

It is known that the fluctuation in the delay time in the HiPIMS discharge is very small so we can assume $t_{\mathrm{s}} \ll t_{\mathrm{f}}$. By treating the initiation of the discharge as a vacuum breakdown Yushkov and Anders ${ }^{138}$ derive an equation for the formative time lag,

$$
t_{\mathrm{f}} \approx \frac{a}{V_{\mathrm{d}}-V_{\mathrm{b}}} \exp \left(\frac{b}{V_{\mathrm{d}}}\right)
$$

where $V_{\mathrm{b}}$ is the static breakdown voltage, $V_{\mathrm{d}}$ is the applied voltage, $a=A p$, and $b=B p d$, where $A$ and $B$ are constants determined experimentally and depend on the gas type and are listed in Table I for common working gases. Poolcharuansin et al ${ }^{134}$ have demonstrated how a dc preionizer can be used to reduce or eliminate the formative time lag and thus the ignition delay time in an HiPIMS discharge when operating at pressures below $0.1 \mathrm{~Pa}$. If the pressure is very low and the delay time is significant the plasma cannot fully develop within the pulse and if the delay is longer than the actual pulse width the plasma will not ignite at all. The role of the preionizer is to provide a seed of charge in the discharge volume between the pulses. This allows for the HiPIMS discharge to be operated at low enough pressure to reach the region of ballistic transport of sputtered particles. ${ }^{139}$ 
TABLE I. Value of the constants $A$ and $B$ for various noble gases. ${ }^{\text {a }}$

\begin{tabular}{lccc}
\hline \hline & $\begin{array}{c}A \\
\text { Gas }\end{array}$ & $\begin{array}{c}B \\
\left(\mathrm{~cm}^{-1} \mathrm{Torr}^{-1}\right)\end{array}$ & $\begin{array}{c}\text { Range of } E / p \\
\left(\mathrm{~V} \mathrm{~cm}^{-1} \mathrm{Torr}^{-1}\right)\end{array}$ \\
\hline $\mathrm{Ne}$ & 4.4 & 111 & $100-400$ \\
$\mathrm{Ar}$ & 11.5 & 176 & $100-600$ \\
$\mathrm{Kr}$ & 15.6 & 220 & $100-1000$ \\
$\mathrm{Xe}$ & 24 & 330 & $200-800$ \\
\hline \hline
\end{tabular}

${ }^{\mathrm{a}}$ From Lieberman and Lichtenberg (Ref. 48).

\section{HIGH POWER IMPULSE MAGNETRON SPUTTERING DISCHARGE}

There has been extensive investigation of the spatial and temporal variation of the plasma parameters in the HiPIMS discharge. A brief overview of the key plasma parameters observed experimentally in an HiPIMS discharge was recently given by Gudmundsson. ${ }^{67}$ Here we give an extended overview of the measured plasma parameters in the HiPIMS discharge, first discussing the electron energy, electron density, and electrical potential in Sec. III A, then the ion energy and plasma composition in Sec. III B, deposition rate in Sec. III C, self-sputtering in Sec. III D, and reactive sputtering in Sec. III E.
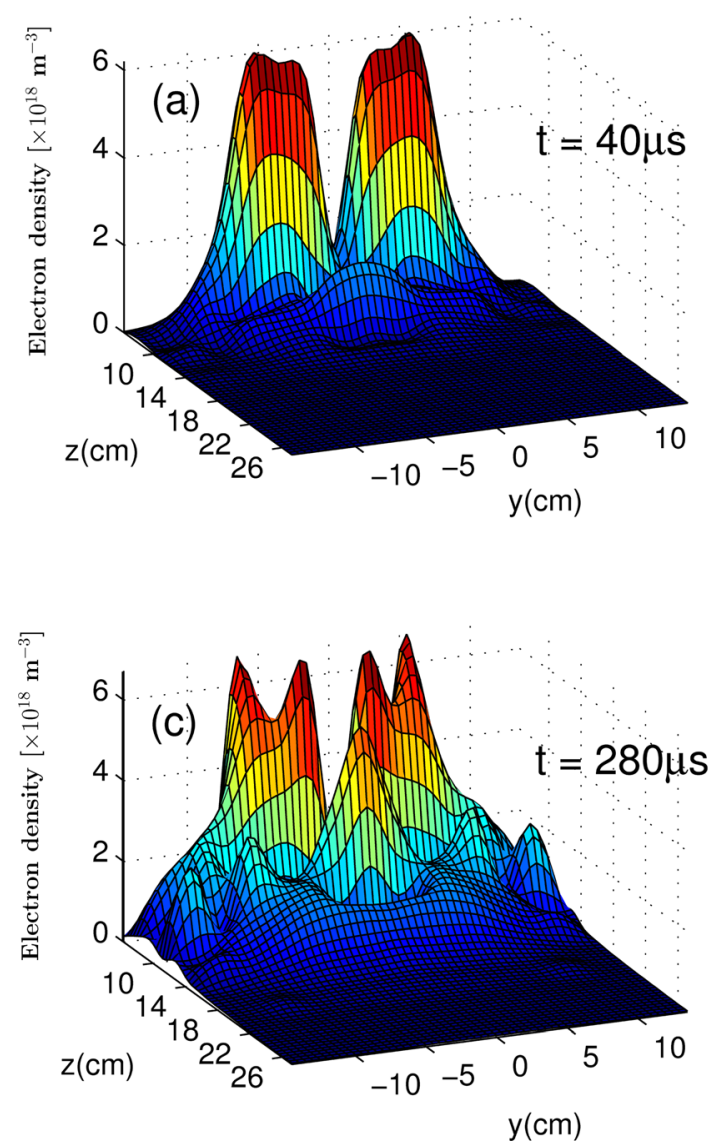

\section{A. Electron energy, electron density, and electrical potentials}

There have been several studies of the spatial and temporal variation of the electron density in the HiPIMS discharge using Langmuir probe diagnostics. ${ }^{94,95,135,140-148}$ Measurements of the temporal and spatial behavior of the plasma parameters indicate a peak electron density of the order of $10^{18}-10^{19} \mathrm{~m}^{-3},{ }^{94,95,140}$ which during the ignition and growth phase expands from the target as an ion acoustic wave, with a fixed velocity that depends on the gas pressure. ${ }^{149}$ This is roughly 2 orders of magnitude higher density than commonly observed in the substrate vicinity for a conventional dcMS discharge. Figure 8 shows the spatial distribution of the electron density in front of a titanium target for various times after initiating the pulse for a gas pressure of $2.7 \mathrm{~Pa}$ (after Bohlmark et al.$^{140,150}$ ). The plasma density remains at a relatively high level of $10^{17}-10^{18} \mathrm{~m}^{-3}$ even $540 \mu \mathrm{s}$ after the pulse is off. In fact a high plasma density $\sim 10^{17}$ is commonly observed to linger at large distances from the target surface for a rather long time, up to a few milliseconds. ${ }^{95,151}$ Also, at larger distances, measurements of the temporal variation of the electron density at higher pressures (above $1.3 \mathrm{~Pa}$ ), show two maxima. ${ }^{95,141}$ A second peak in the electron density appears several hundred microseconds after the end of the high voltage pulse. ${ }^{95}$ The timing of this second
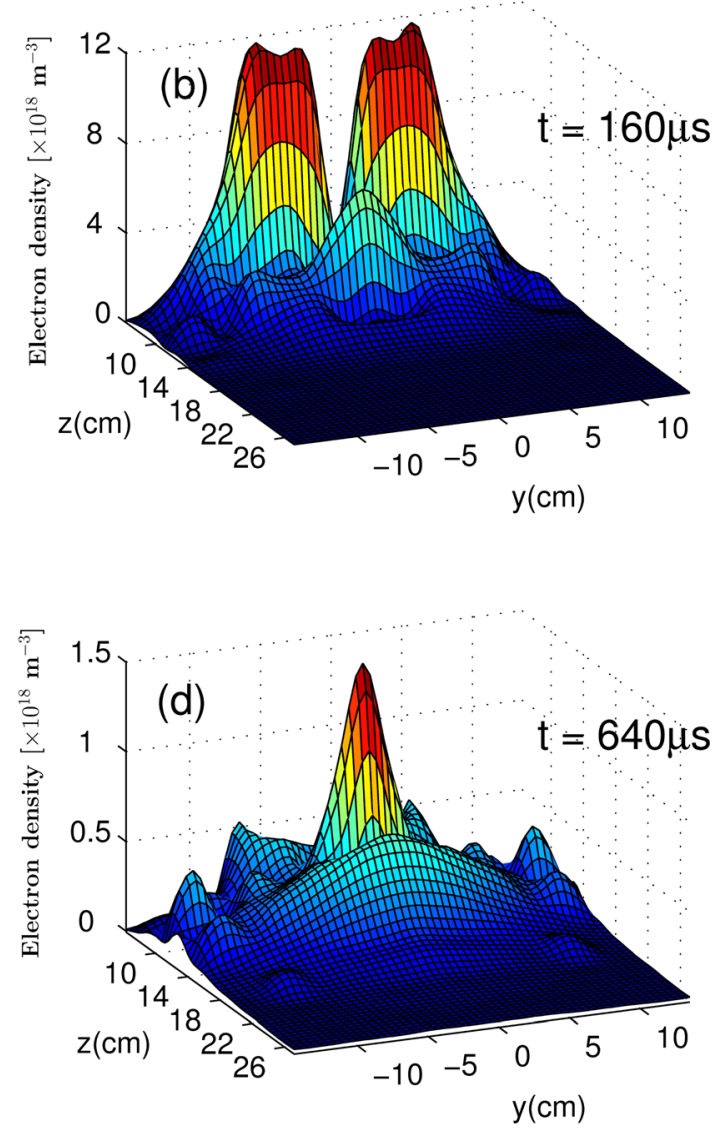

FIG. 8. (Color online) Temporal and spatial variation of the electron density. The snapshots are taken (a) 40, (b) 160 , (c) 280 , and (d) $640 \mu$ s after initiating the $100 \mu \mathrm{s}$ long pulse. The gas pressure was $2.7 \mathrm{~Pa}$, pulse energy $9 \mathrm{~J}$, and the target made of Ti $150 \mathrm{~mm}$ in diameter. After Bohlmark et al. (Refs. 140 and 150 ). 
peak depends on the chamber dimension, and it is thus believed to be due to reflections of neutrals from the chamber walls (the existing plasma will be locally densified by the reflection). ${ }^{141}$ Vetushka and Ehiasarian ${ }^{142}$ report a factor of 2 higher electron density in the substrate vicinity for a discharge with a chromium target than for a titanium target for the same discharge current. They also report mass spectrometry measurements that show the metal argon ratio for chromium $n_{\mathrm{Cr}^{+}} / n_{\mathrm{Ar}^{+}}$is roughly twice of the ratio $n_{\mathrm{Ti}^{+}} / n_{\mathrm{Ar}^{+}}$for the same discharge current. ${ }^{152,153}$ In a reactive $\mathrm{Ar} / \mathrm{O}_{2}$ discharge the electron density peak value, slightly below $10^{18} \mathrm{~m}^{-3}$, is roughly independent of the discharge pressure but the peak electron density increases with increased discharge current. ${ }^{147}$ MPP sputtering uses relatively long pulses (a few milliseconds) of high, but not extreme power densities, and allows the operator to play with the voltage waveform, e.g., apply a stepwise increase or decrease in the applied power. ${ }^{20,25,119}$ Because of this limited peak power the peak plasma density is in the range $10^{17}-10^{18} \mathrm{~m}^{-3},{ }^{154}$ which is lower than is observed for the shorter and more intense HiPIMS pulses as discussed earlier. In summary, it is seen that the HiPIMS discharge generates a dense plasma both in metal as well as reactive mode with peak densities reaching up to $10^{19} \mathrm{~m}^{-3}$. Generally a monotonic rise in plasma density with discharge gas pressure ${ }^{95}$ and applied power ${ }^{141}$ and linear increase in electron density with increased discharge current, ${ }^{144}$ is observed.

For dcMS the electron energy distribution function (EEDF) is commonly observed to be bi-Maxwellian in the substrate vicinity ${ }^{61,63,155}$ Sheridan et al. ${ }^{61}$ claim that the hot electrons are not energetic enough to be the ones emitted from the target, but are created in the magnetic trap in the cathode fall regions and drift to the downstream region under the influence of a diverging magnetic field. ${ }^{60,155}$ For the HiPIMS discharge the EEDF during the pulse has been observed to be Maxwellian. ${ }^{135}$ However, bi-Maxwellian behavior, with weakly populated hot electrons, has been reported for the initial stages of the pulse. ${ }^{143}$ Poolcharuansin and Bradley ${ }^{146}$ claim that there are three distinct groups of electrons in the initial stages of the pulse $(<4 \mu \mathrm{s})$. They describe these three distinct groups as super-thermal, hot, and cold electrons with effective electron temperatures in the ranges $70-100,5-7$, and $0.8-1 \mathrm{eV}$, respectively. The explanation given by Poolcharuansin and Bradley ${ }^{146}$ for the existence of the super-thermal electrons assumes that initially the sheath is very thin or nonexisting depending on the offtime remnant plasma. After the pulse initiation the sheath expands rapidly due to increasing target cathode voltage. The electrons from the remnant off-time plasma then get accelerated in the increasing axial field of the forming sheath. This results in an $\mathbf{E} \times \mathbf{B}$-drift and azimuthal current. As the sheath edge advances these electrons may then bounce from the leading edge of the sheath, similar to what is referred to as stochastic heating in capacitively coupled $\mathrm{rf}$ discharges. ${ }^{49}$ Thus, beamlike electrons are formed and are detected as a very high effective electron temperature in the first few microseconds after the voltage initiation. This leads to a very high negative floating probe potential as has been observed by Pajdarová et al. ${ }^{143}$ and Poolcharuansin and Bradley. ${ }^{146}$ The temporal variation of the effective electron temperature shows generally relatively high values $(2-6 \mathrm{eV})$ about a few tens of microseconds into the pulse, which then decrease to values of $0.3-1 \mathrm{eV} .{ }^{135,142,144,145}$ Vetushka and Ehiasarian $^{142}$ report a peak electron temperature of $2.3-7 \mathrm{eV}$, depending on the pressure and target material, early in the pulse and then a relatively constant value of 0.4 and $0.8 \mathrm{eV}$ after the pulse is off for at least $300 \mu \mathrm{s}$ at $0.3 \mathrm{~Pa}$, for $\mathrm{Cr}$ and $\mathrm{Ti}$ targets, respectively. Similar findings were reported by Gudmundsson et al., ${ }^{135}$ which see the effective electron temperature fall before the end of the pulse to a constant value of about $0.7 \mathrm{eV}$ at $0.4 \mathrm{~Pa}$ and $0.3-0.4 \mathrm{eV}$ at 2.7 Pa. Electron temperatures of $1.5-2 \mathrm{eV}$ a few tens of microseconds into the pulse that cool off during the pulse have also been observed in a reactive $\mathrm{Ar} / \mathrm{O}_{2}$ discharge. ${ }^{147} \mathrm{By}$ optical emission spectroscopy Ross et al. ${ }^{156}$ find a stable well-defined electron temperature of roughly $1.2 \mathrm{eV}$ from $20 \mu$ s after the plasma ignition to the end of the pulse when sputtering from a titanium target in argon at $0.67 \mathrm{~Pa}$. The electrons are expected to be cooled down more effectively in the HiPIMS discharge since there is a significantly higher density of metal species during the pulse (but not necessarily on average) in an HiPIMS discharge compared to a conventional dcMS discharge, and electron impact excitation and ionization of the metal atoms have lower thresholds than the inert working gas. During the initial stages of the pulse the ions of the inert working gas dominate the discharge, while later in the pulse metal ions build up and become the abundant ion species (see Sec. III B).

The spatial and temporal variation of the plasma potential has been measured by an emissive probe in an argon discharge with titanium target at $0.54 \mathrm{~Pa}^{157}$ These measurements show that in the initial stages of the $100 \mu$ s pulse ( $\sim 6-8 \mu$ s into the pulse) the plasma potential exhibits very deep negative values $(-150 \mathrm{~V})$ next to the target $(1 \mathrm{~cm}$ away). This value decreases with distance, but does never reach ground potential. Within the confined plasma region Mishra et al. ${ }^{157}$ also find extraordinarily high axial and radial electric field components, or up to several $\mathrm{kV} / \mathrm{m}$, calculated from the plasma potential measurements. As the plasma develops and the discharge current reaches a maximum (roughly $40 \mu$ s into the pulse), the plasma potential is elevated everywhere within the plasma, but deep values (down to $-40 \mathrm{~V}$ ) remain at positions closest to the target surface $(z=1 \mathrm{~cm})$.

There are also reports on characterization of multiple magnetron systems that allow for additional control parameters, such as the delay between subsequent pulses. Stranak et al. ${ }^{158}$ performed time resolved Langmuir probe measurements in a dual HiPIMS system with two cathodes in a closed magnetic field configuration. They explored the effect of a delay between the subsequent pulses and demonstrate that the delay can be applied as an additional control parameter to adjust the process parameters. The peak electron density during the second pulse as well as the temporal variation of the effective electron temperature and the ion fluxes on the substrate can be controlled by the delay time between the 
pulses. Vozniy et al. ${ }^{159}$ apply high power pulses to four cathode targets. They find that if a $20 \mu$ s long pulse is applied first to two magnetron targets, and then $20 \mu$ s later to the second pair of cathode targets, the ion density is about $40 \%$ higher than when applying the pulse simultaneously to all four cathode targets. In this experiment the average power was $200 \mathrm{~W}$ and the repetition frequency $1 \mathrm{kHz}$.

\section{B. Ion flux, energy, and composition}

In dcMS, the sputtered vapor is considerably more energetic than thermally evaporated atoms (a few $\mathrm{eV}$ as compared to about a tenth of an $\mathrm{eV}$ ). Usually it is desirable to maintain this initial kinetic energy of the sputtered atoms, because of its effect on the film growth. ${ }^{160}$ Relatively low pressures are normally used to minimize scattering of the sputtered atoms. The sputtering process is, therefore, normally a line-of-sight process where the deposition flux cannot be easily controlled, since it consists of neutral atoms. The ion energy distribution of the ions of the sputtered vapor in a dcMS generally shows a narrow low energy peak, due to thermalized ions that are accelerated by the plasma potential, and a broad distribution at higher energies that originates from the sputtered neutrals which have been ionized by electron impact within the plasma (see, e.g., Ref. 161). Due to the small mass of the electron the electron impact ionization does not change the energy of the resulting ion by much. This broad distribution has been measured for sputtered neutrals ${ }^{162}$ and is predicted by the Thompson random collision cascade model. ${ }^{163,164}$ According to the Sigmund-Thompson theory the energy distribution function can be approximated by

$$
f_{\mathrm{S}-\mathrm{T}} \propto \frac{\mathcal{E}}{\left(\mathcal{E}+\mathcal{E}_{\mathrm{b}}\right)^{3}},
$$

where $\mathcal{E}_{\mathrm{b}}$ is the binding energy of the target material. ${ }^{164-166}$ This model predicts an energy spectrum that peaks sharply at $\frac{1}{2} \mathcal{E}_{\mathrm{b}}$, followed by a gradual decrease to higher energies $\left(\propto 1 / \mathcal{E}^{2}\right)$.

The first measurements of the ion energy distribution function (IEDF) in an HiPIMS discharge were reported by Bohlmark et al. ${ }^{167}$ for a titanium target sputtered with argon as the working gas at $0.4 \mathrm{~Pa}$. The mass spectrometer was inserted from the side of the chamber, parallel to the target surface. The ion energy for $\mathrm{Ti}^{+}$ions showed a high energy tail with ions traced to an energy up to $100 \mathrm{eV}$. The average ion energy was roughly $20 \mathrm{eV}$. Furthermore, a highly metallic ion flux was observed during the active phase of the discharge. The energetic metal ions are mainly created during the pulse, while the target current reaches its maximum. For comparison the ion energy distribution from a dcMS has a peak ion energy of about $2 \mathrm{eV}$ and high energy tail that extends to 20 and $40 \mathrm{eV}$ for $\mathrm{Ar}^{+}$and $\mathrm{Ti}^{+}$ions, respectively. This is demonstrated in Fig. 9, which shows a comparison of the ion energy distribution for the $\mathrm{Ti}^{+}$ion from a HiPIMS discharge and dcMS discharge with titanium target (from Ref. 168). Ehiasarian et al. ${ }^{152}$ report somewhat lower maxi-

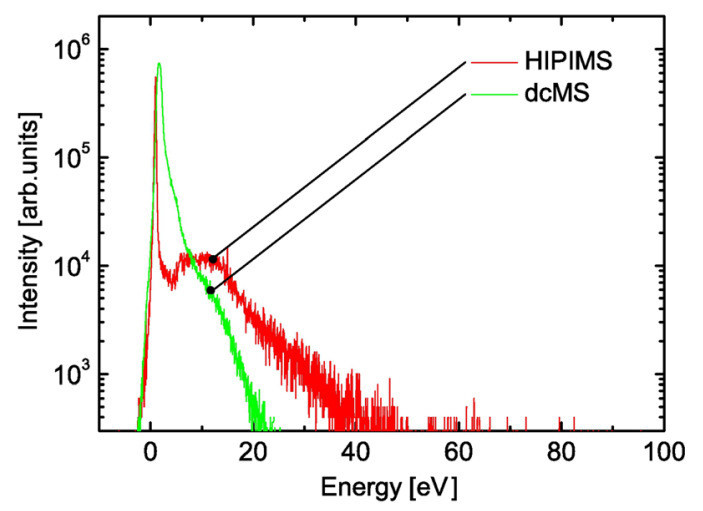

FIG. 9. (Color online) Time averaged ion energy distribution for $\mathrm{Ti}^{+}$ions from HiPIMS and dcMS discharges measured at $0.80 \mathrm{~Pa}$ and $1 \mathrm{~cm}$ below the target using equal average power. Note that the displayed intensities are in arbitrary units and that no correlation between the different abundances of $\mathrm{Ti}^{+}$can be made based on this measurement only. Furthermore, the distributions have been normalized to fit into one single plot. The mass spectrometer was inserted from the side of the chamber (i.e., parallel to the target surface). From Lundin et al. (Ref. 168).

mum energy for the $\mathrm{Ti}^{+}$ions or $45 \mathrm{~V}$ at $0.4 \mathrm{~Pa}$, which may be due to a different geometry of the experiment. $\mathrm{Ti}^{+}$ions deflected sideways from the magnetron target were investigated further by Lundin et al. ${ }^{168}$ They observe a high energy tail that extends up to $80 \mathrm{eV}$ when the mass spectrometer is perpendicular to the cathode target surface but only up to $23 \mathrm{eV}$ when the mass spectrometer is perpendicular to but in a plane $5 \mathrm{~cm}$ from the target surface. This is shown in Fig. 10. Furthermore, in the same experiment when the orifice of the mass spectrometer was facing two opposite sides of the target race track, it was seen that the high energy tail reached about $60 \mathrm{eV}$ from one side, but only about $40 \mathrm{eV}$ from the other side. This azimuthal kick of the ions is consistent with the forces exerted by the azimuthally rotating current below the circular magnetron, which tangentially ejects the ions sideways with an asymmetric ion energy distribution. Independent corroboration of this result was recently presented by Poolcharuansin et al. ${ }^{169}$ who used a retarding field analyzer to measure the velocity and energy distribution functions of ions ejected sideways out of an

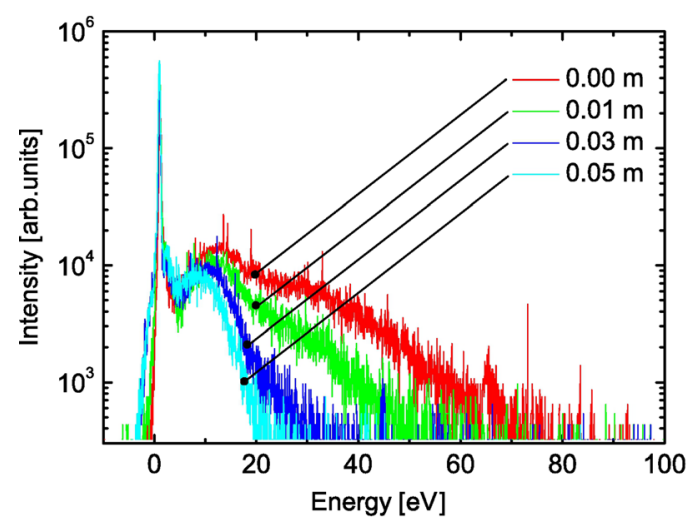

FIg. 10. (Color online) Time averaged ion energy distribution for $\mathrm{Ti}^{+}$ions at $0.53 \mathrm{~Pa}$ using $500 \mathrm{~V}$ HiPIMS pulses for different distances from the target surface. The average ion energy is found to be around $18 \mathrm{eV}$ when measured at the target position. The mass spectrometer was inserted from the side of the chamber (at $z=0.00 \mathrm{~m}$ ). From Lundin et al. (Ref. 168). 
HiPIMS discharge. This revealed high-energetic tails with ion energy measured up to $50 \mathrm{eV}$, with the ion flux and the mean energy of the ions distributed asymmetrically; the maxima were obtained into the direction of an oncoming ion fluid rotating in the same direction as the electron $\mathbf{E} \times \mathbf{B}$ drift. The physical mechanism for the origin of this behavior is further discussed in Sec. IV D. With a mass spectrometer that faces the target Vlček et al. observed copper ions that have energies of up to $45 \mathrm{eV},{ }^{170}$ and titanium ions that have energies of up to $50 \mathrm{eV},{ }^{171}$ at $0.6 \mathrm{~Pa}$, and zirconium ions with energies up to $100 \mathrm{eV}$ at $1 \mathrm{~Pa}^{172}$ They find $\mathrm{Cu}^{+}$ions to be up to $80 \%-95 \%$ of the total ion flux to the substrate. ${ }^{173}$ Mishra et al. ${ }^{174}$ also have the mass spectrometer facing the race track region of the target $10 \mathrm{~cm}$ from a titanium target. They find $\mathrm{Ti}^{+}$ions having a high energy tail extending up to $100 \mathrm{eV}$.

Hecimovic et al. ${ }^{153,175,176}$ explore the IEDFs from $\mathrm{Cr}$, Ti, and $\mathrm{C}$ targets. The average energy of metal ions was higher for $\mathrm{Ti}^{+}$ions than for $\mathrm{Cr}^{+}$ions, while $\mathrm{C}^{+}$ions had the lowest mean energy for a given target current. With a mass spectrometer facing the center of the magnetron target they also explored the temporal evolution of ions at various distances from the target and found that the plasma composition and the IEDF depend strongly on the distance from the target surface, and that the sputtered ion-to-working gas ion flux ratio is in the range from 1 to $3.5 .{ }^{176}$ The IEDFs of the ions of the sputtered vapor consist of two Maxwellian distributions with low and high energy groups of ions. ${ }^{153}$ They attribute the low energy group to fully thermalized ions of the sputtered vapor that exist in the post-discharge and claim that the high energy tail originates from the pulse on period and shortly after the end of the pulse ${ }^{175}$ and originates from the sputtering cascade, by electron impact ionization of the sputtered vapor atoms which follow the Thompson distribution. ${ }^{163}$ They also find that the average ion energy increases with increasing peak discharge current. The ions of the working gas exhibit lower energy but still have a high energy tail. They also observed that the metal ions have a lifespan of a few milliseconds, the actual lifespan depending on the target material, where light elements, such as $\mathrm{C}$ and $\mathrm{Al}$ lose more energy in collisions with $\mathrm{Ar}$ atoms and thereby have a shorter lifetime than, for example, $\mathrm{Nb}$, and that the lifespan of argon ions is longer than that of the metal ions. ${ }^{137,175}$ Also, in the time off phase only thermalized low energy ions are observed and the ion flux decays exponentially with time.

At pressures below $0.1 \mathrm{~Pa}$ the sputter deposition is essentially a collisionless line-of-sight deposition process. ${ }^{93}$ Poolcharuansin and Bradley ${ }^{139}$ use a preionizer to shorten the ignition time while sputtering a carbon target with argon as the working gas at a very low pressure $(86 \mathrm{mPa})$. For the argon ions the main peak is at roughly $0 \mathrm{eV}$ with a secondary feature at $\sim 5-10 \mathrm{eV}$. The carbon ions exhibit two distinct peaks in the ion energy distribution, one at low energy $(\sim 0 \mathrm{eV})$ and another at higher energy $(\sim 10 \mathrm{eV})$. The higher energy peak is of much higher intensity than the lower energy peak. They find that the high energy peak was created during the on-time phase of the HiPIMS pulse, while the low energy peak dominated the ion energy distribution during the off time. Thus the high energy peak reflects the energy of the sputtered atoms as they are ejected from the target (the Thompson distribution). When the operating pressure was increased the intensity and the energy of the energetic peak decreased. They claim that for the low pressure condition the energetic ions are transported ballistically to the orifice of the mass spectrometer and suggest that this allows for a condition of a collision-free operation.

Quantitative measurement of the metal ion to metal atom ratio is rare. Bohlmark et al. ${ }^{177}$ measured the degree of fractional ionization of the sputtered vapor to be over $90 \%$ for an argon discharge with titanium target using optical emission spectroscopy (OES). Measurement of the ionized flux fraction has also been made using weight gain differences on a floating and a positively biased substrate. In this way, the ionized flux fraction for $\mathrm{Cu}$ and $\mathrm{Cr}$ has been estimated to be roughly $70 \%$ (Ref. 16) and $30 \%,{ }^{178}$ respectively. The same group estimated the degree of metal ionization of the flux from a $\mathrm{Ti}_{0.5} \mathrm{Al}_{0.5}$ target to be around $40 \%{ }^{17}$ Using a quartz crystal microbalance mounted behind a two layer-gridded energy analyzer, the degree of ionization of $\mathrm{C}$ and $\mathrm{Al}$ during sputtering was measured as $4.5 \%$ and $9.5 \%$, respectively. ${ }^{179}$ These values are significantly lower than those reported by the other groups. For C, a lower ionization fraction is expected since it has a higher ionization potential and a smaller electron impact cross section as compared to metals. Recently, it has been demonstrated that using neon as the working gas the electron temperature can be elevated when sputtering carbon target that leads to higher ionization fraction of the sputtered vapor. ${ }^{180}$ Keep in mind that for a weakly ionized discharge the electron temperature is much larger than the neutral gas temperature or $T_{\mathrm{e}} \gg T_{\mathrm{g}}$ so that the ion flux fraction for the sputtered vapor is larger than the ionization fraction of the sputtered vapor in the plasma. Thus, it is not necessary to completely ionize the sputtered vapor to create a highly ionized flux to the substrate..$^{9,67}$

There have also been several studies of the ion composition and ion energy distribution in reactive HiPIMS discharge, including titanium target in an $\mathrm{Ar} / \mathrm{O}_{2}$ discharge ${ }^{181}$ and $\mathrm{Ar} / \mathrm{N}_{2}$ discharge, ${ }^{40,152,182}$ aluminum in an $\mathrm{Ar} / \mathrm{N}_{2}$ discharge, ${ }^{183}$ and chromium in an $\mathrm{Ar} / \mathrm{N}_{2}$ discharge in an industrial size reactor. ${ }^{184}$ Measurements of the IEDF of $\mathrm{Ti}^{+}$and $\mathrm{O}^{+}$ions in an $\mathrm{Ar} / \mathrm{O}_{2}$ discharge while sputtering $\mathrm{Ti}$ target show a main peak at low energy and a shoulder extending to a high energy tail for both species. ${ }^{181}$ The high energy tail extends to higher energy for $\mathrm{O}^{+}$compared to $\mathrm{Ti}^{+}$. Furthermore, the energy of both $\mathrm{Ti}^{+}$and $\mathrm{O}^{+}$ions increased with increasing peak power (while the average power was kept fixed), while $\mathrm{Ar}^{+}$only exhibit energy in the few $\mathrm{eV}$ range, for a $35 \mu$ s long pulse and $100 \mathrm{~W}$ average power. For the highest peak power explored the energy of the $\mathrm{O}^{+}$ions extendend to $100 \mathrm{eV}$, while the tail for the $\mathrm{Ti}^{+}$-ions extended to $60 \mathrm{eV}$. Greczynski and Hultman ${ }^{184}$ report on time and energy resolved mass spectrometry in an industrial deposition system for both pure argon and reactive $\mathrm{Ar} / \mathrm{N}_{2}$ sputtering of a $\mathrm{Cr}$ target. The IEDFs of $\mathrm{Cr}^{+}$ions comprise an intense low energy peak and pronounced high energy tails and the number of ions in the high-energy portion of the IEDF increases with increased pulse energy. The majority of 
$\mathrm{Ar}^{+}$ions are thermalized and the IEDF comprises an intense peak around $2-3 \mathrm{eV}$. The intensity of the $\mathrm{Ar}^{+}$signal does not depend on the pulse energy and the $\mathrm{Ar}^{+}$ions clearly precede the $\mathrm{Cr}^{+}$ions. During reactive sputtering in $\mathrm{Ar} / \mathrm{N}_{2}$ discharges, low energy $\mathrm{N}_{2}^{+}$-ions and energetic $\mathrm{N}^{+}$ions are present in reactive mode. The IEDF of the $\mathrm{N}^{+}$ion possesses a highenergy tail just like the $\mathrm{Cr}^{+}$ions (or the $\mathrm{Cr}^{2+}$ ions), which is not observed for $\mathrm{Ar}^{+}$or $\mathrm{N}_{2}^{+}$. Furthermore, there is a time synchronization between the $\mathrm{Cr}^{+}$and $\mathrm{N}^{+}$signals. The initial phase is always dominated by relatively low-energy ions of the working gas $\left(\mathrm{Ar}^{+}\right.$or $\left.\mathrm{Ar}^{+}, \mathrm{N}_{2}^{+}\right)$. This is followed by a phase dominated by the ions of the sputtered species $\left(\mathrm{Cr}^{+}\right.$or $\left.\mathrm{Cr}^{+}, \mathrm{N}^{+}\right)$. Finally, thermalized ions of the working gas dominate. Similar findings have been reported for an $\mathrm{Ar} /$ $\mathrm{N}_{2}$ discharge with a titanium target ${ }^{40,152}$ and Ehiasarian et al. ${ }^{182}$ find the metal ion-to-metal neutral ratio $n_{\mathrm{Ti}^{+}} / n_{\mathrm{Ti}}$ as well as the atomic ion to molecular ion ratio $n_{\mathrm{N}} / n_{\mathrm{N}_{2}^{+}}$to be directly proportional to the peak discharge current. Also Jouan et al. ${ }^{183}$ find that $\mathrm{N}^{+}$and $\mathrm{Al}^{+}$ions have a high energy tail and that the discharge is dominated by $\mathrm{Al}^{+}$ions while sputtering $\mathrm{Al}$ target in $\mathrm{Ar} / \mathrm{N}_{2}$ discharge.

There have been several reports on the presence of multiply charged ions in the HiPIMS discharge. ${ }^{117,134,144,152,168,172,176,184-186}$ As will be discussed in Sec. IIID, multiply charged ions are essential for the maintainence of the discharge when self-sputtering dominates. Bohlmark et al. ${ }^{168}$ report that for a Ti target a significant fraction of the ion flux is the $\mathrm{Ti}^{2+}$ ion and an observation of the $\mathrm{Ti}^{4+}$ ion has been reported. ${ }^{134,186}$ Poolcharuansin et al.$^{133}$ claim that there is an increase in multiply charged ions with decreasing operating pressure, in particular in the low pressure regime below $0.5 \mathrm{~Pa}$.

Greczynski and Hultman ${ }^{184}$ find that the number of doubly charged $\mathrm{Cr}^{2+}$ ions increased almost linearly with increased pulse energy in an $\mathrm{Ar} / \mathrm{N}_{2}$ industrial scale discharge. Lazar et al. ${ }^{172}$ see a significant fraction of doubly charged zirconium ions in the total ion flux. The flux fraction of doubly charged zirconium ions was higher than the fraction of singly charged zirconium ions. It increased with increased distance from the cathode target, and could become strongly dominant. In their experiment the mass spectrometer was located on the target substrate axis facing the target surface of a strongly unbalanced magnetron source. As discussed by Ross et al. ${ }^{156}$ there is an optimum condition for the HiPIMS discharge where the maximum number of singly charged ions are created per unit energy. They argue that the sputtering efficiency decreases as energy is wasted, creating the more highly ionized species and that the multiply charged species are created by secondary electrons.

As discussed earlier, this high ionization fraction and high ion flux to the substrate have significant influence on the overall quality of the growing film. ${ }^{7,8,187}$ The benefits of high ion flux to the substrate are even more pronounced when depositing on a complex substrate, where deposition on substrate such as drills and cutting inserts is of utmost importance for industrial applications. For films grown on substrates that are orthogonal to the target surface the line-of-sight deposition typically leads to film columns aligned in the direction of the incoming flux. It has been demonstrated that column tilt in Ta films grown on surfaces orthogonal to the target can be eliminated by using HiPIMS. ${ }^{41}$ As films grown by dcMS showed pronounced columns aligned toward the direction of incident material flux, the films grown by HiPIMS had columns growing normal to the surface. This effect has been further explored by Greczynski et al. ${ }^{188}$ for the growth of $\mathrm{Cr}$ films. They show that the $\mathrm{Cr}$ ion-to-neutral ratio in the flux incident onto the substrate depends on the peak target current density. They find that the column alignment commonly observed for growth at low ion-to-neutral ratio decreases with increasing ion content in the flux and that column tilting can be almost eliminated at high values of ion-to-neutral ratio. When the material flux to the substrate is highly ionized the deposition takes place along the substrate normal despite the high inclination angle, and the growth is mostly independent of the substrate orientation. Alami et al. ${ }^{27}$ demonstrated an equal deposition rate of TiAlN coating on the flank and rake faces of a cutting insert when depositing in an industrial system with four magnetrons, in the CFUBMS configuration, using a combination of dc and HiPIMS power supplies and a rotating substrate holder. Also Bohlmark et al. ${ }^{39}$ demonstrated, that due to a high degree of ionization of the sputtered vapor, the spatial distribution of material flux during HiPIMS can be steered when using an external magnetic field. Furthermore, the ion bombarding energy at the substrate can be controlled by a bias voltage. Note that the ion currents during the pulse can be high and the bias voltage supply has to be able to handle the high currents. Bugaev and Sochugov ${ }^{131}$ control the ion bombarding to the film substrate by a high-voltage bias that is synchronized with the pulsed power supply of the magnetron discharge.

There have been reports of significantly lower heat transfer to the substrate in HiPIMS compared to dcMS. This has a significance in particular when depositing on temperature sensitive substrates. Both Lundin et al. ${ }^{189}$ and West et al. ${ }^{190}$ claim that the substrate heating is severely reduced in the planar HiPIMS discharge compared to conventional dcMS discharge at the same average power. However, the energy flux per deposited particle is higher for an HiPIMS discharge compared to a dcMS discharge. It is also pointed out that heating due to radial energy flux reached as much as $60 \%$ of the axial energy flux. ${ }^{189}$ However, for a rotating cylindrical magnetron sputtering discharge with a titanium target Leroy et $a l .{ }^{191}$ find that the total energy flux arriving at the substrate is slightly lower for HiPIMS than for dcMS for the same average power, but that the difference is smaller for shorter pulse length. In addition, from absolute temperature measurements at a common substrate position during an HiPIMS process it was found that the maximum equilibrium temperature reached about $70^{\circ} \mathrm{C}$ using an average power of $500 \mathrm{~W}$ on a $150 \mathrm{~mm}$ diameter Ti target and a peak current density of about $1.5 \mathrm{~A} \mathrm{~cm}^{-2}$. ${ }^{189}$

\section{Deposition rate}

The deposition rate is generally found to be lower in an HiPIMS discharge than in a conventional dcMS discharge at 
the same average power. Sputtering rates are determined by the ion current density at the target and the sputter yield, which is determined by the target material, the bombarding species, and the bombarding ion energy (the target voltage). Sputtering is most conveniently described in terms of average effects or by sputter yield $Y$, which is defined as the number of atoms ejected by sputtering per incident projectile. The deposition rate depends on the sputtering rate. For the dcMS the deposition rates are practically found to be directly proportional to the power applied to the target. ${ }^{3}$ The deposition rates are thus determined by the power density, target material, size of the erosion area, target-to-substrate distance, and discharge pressure. In the HiPIMS regime the absolute deposition rate increases almost linearly with increasing applied power, while the deposition rate per unit power decreases. ${ }^{156}$ The early investigations on the high power pulsed sputtering reported on lower deposition rate, a factor of 2 lower for both $\mathrm{Cu}$ and Ti thin films. ${ }^{114}$ This was further confirmed by comparing the deposition rates of various metals ( $\mathrm{Ti}, \mathrm{Cr}, \mathrm{Zr}, \mathrm{Al}, \mathrm{Cu}, \mathrm{Ta}, \mathrm{Pt}, \mathrm{Ag}$ ) for both dcMS and HiPIMS discharges applying the same average power. ${ }^{31}$ The deposition rate for the HiPIMS discharge is lower than the deposition rate for dcMS in all cases as seen in Fig. 11. However, the reduction in the deposition rate was not more pronounced for materials with low sputtering yield as had been concluded from measured data. ${ }^{23}$ Konstantinidis et al. ${ }^{192}$ find that the deposition rate depends on the pulse length and increases from $20 \%$ to $70 \%$ of dcMS values as the pulse length is decreased from 20 to $5 \mu$ s for the same average power of $300 \mathrm{~W}$ when sputtering a titanium target in argon at $1.33 \mathrm{~Pa}$. For a rotating cylindrical magnetron Leroy et al. ${ }^{191}$ find the deposition rate to be up to $75 \%$ lower for HiPIMS compared to dcMS, and the decrease in deposition rate becomes more pronounced with increased pulse length. This may be due to the fact that the shorter pulse length does not allow the gas rarefaction, self-sputtering and other processes to develop (these mechanisms will be discussed in more detail in Sec. IIID as well as in Sec. IV). In short, HiPIMS pulses $\left(t_{\mathrm{on}}<50 \mu \mathrm{s}\right) \mathrm{Ar}^{+}$ions are the dominant sput-

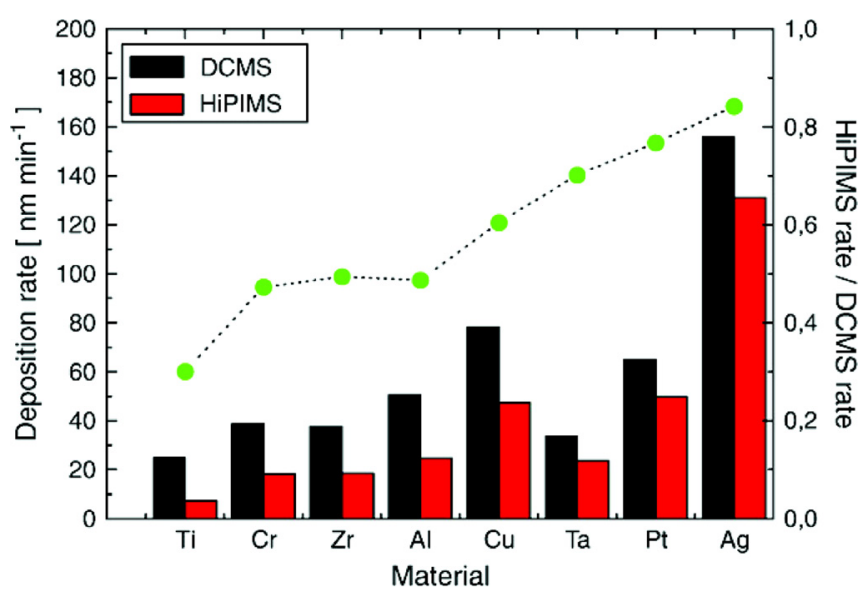

FIG. 11. (Color online) Deposition rates for dcMS and HiPIMS discharges plotted as bars for the different target materials used (left axis). The deposition rate of HiPIMS over dcMS deposition rate is shown as a scatter plot (right axis). From Samuelsson et al. (Ref. 31). tering particles, ${ }^{193}$ which means that very little selfsputtering is taking place and, thus, that the effective sputter rate can be kept high, as discussed in Sec. IV A. In general, the deposition rate in the self-sputtering mode is found to be lower than when argon sputtering is dominating. ${ }^{194}$

There have been several suggestions on the cause of the lower deposition rate. One explanation for the reduction in deposition rate is that the sputtered material is ionized close to the target. Alternatively, the negative potential can extend far into the plasma as an extended presheath. In both cases many of the metal ions will be attracted back to the target surface by the cathode potential. ${ }^{195}$ This is often referred to as back attraction or the returning ion effect. A reduction in the deposition rate would then be more pronounced for metals with a low self-sputter yield and for metals with a high degree of ionization. ${ }^{11,23}$ A phenomenological model describing this process will be discussed in Sec. IV A. The target material (or the target binding energy) mainly determines the sputter yield and it depends only slightly on the type of ion bombarding the target. The curves for sputter yield versus ion energy for argon ions and self-ion sputtering for various target materials are very similar; however, they are not identical and the self-sputter yield is typically $10 \%-15 \%$ lower. ${ }^{196} \mathrm{In}$ addition, the projectile particle is lost and that decreases the "effective yield" by 1 , which reduces the sputtered material flux considerably. Also, as the primary sputtering ion changes from the ion of the sputtering gas to self-sputtering, a change in the secondary electron emission yield is expected, in particular at the relatively low ion bombarding energy applied in magnetron sputtering $\mathcal{E}_{\mathrm{i}}<1 \mathrm{keV} .{ }^{117,197}$ As a rule of thumb, the secondary electron yield decreases as the incoming projectile changes from a gas ion to a metal ion $\left(\gamma_{\mathrm{SE}} \approx 0.1\right.$ for $\mathrm{Ar}^{+}$while $\gamma_{\mathrm{SE}} \approx 0$ for $\mathrm{M}^{+}$). As discussed in Sec. IV B, gas rarefaction leads to lower density of the working gas in front of the target and thus a reduction in the number of ions available for sputtering. This subsequently leads to a reduction in the deposition rate.

Emmerlich et al. ${ }^{198}$ argue that when comparing dcMS and HiPIMS discharges at the same average power the nonlinear scaling of the sputter yield with the applied voltage is not taken into account (often $Y \sim \sqrt{V_{\mathrm{d}}}$ ). This would reduce the sputtering rate since in HiPIMS operation the target voltage is significantly higher than for a conventional dcMS discharge, so it is not reasonable to compare the two at the same average power. Alami et al. ${ }^{199}$ explain the lowered deposition rate partially by a lower average target current during HiPIMS deposition when the same average power is applied due to higher voltage necessary for HiPIMS operation, and thus comparison should be made for the same average current. They also find up to $40 \%$ higher deposition rate for HiPIMS than for dcMS for the same average current when depositing $\mathrm{TiO}_{x}$ films from a $\mathrm{TiO}_{1.8}$ target. ${ }^{35}$ Aiempanakit et al. ${ }^{181}$ find that the deposition rate decreases with increased peak power (lower repetition frequency) when the average power is kept fixed ( $35 \mu$ s long pulse and $100 \mathrm{~W}$ average power) and only a marginal loss in deposition rate compared to dcMS is observed for repetition frequency of 4 $\mathrm{kHz}$ when depositing $\mathrm{TiO}_{2}$ films. 
It has been claimed that the magnetic confinement influences the deposition rate. ${ }^{39,114}$ Mishra et al. ${ }^{200}$ explored the effects of the magnetic field strength on the deposition rate. They demonstrate that by lowering the mean magnetic field in the trap region (by 33\% at the target surface) the deposition rate increased by up to a factor of 6 . They relate the increase in deposition rate to a decrease in the effective potential barrier in the trap region, which hampers the ions leaving the trap. This is also seen as reduced drop in potential over the trap region as the magnetic field strength is reduced. Controlling and optimizing the potential profile in the cathode region will therefore greatly affect the number of metal ions incident on the target surface, which has so far not been carried out. Similarly, an increase in deposition rate with decreasing magnetic field strength has been observed for an MPP discharge in a thorough study that included various target materials. ${ }^{201}$ However, this increase in deposition rate was accompanied by a decrease in ionization fraction of the sputtered vapor and influenced the microstructure and properties of the resulting films. Lundin et al ${ }^{167}$ show that a significant fraction of the sputtered metal species is deposited sideways. This enhanced radial transport (across the magnetic field lines) increases the deposition rates perpendicular to the target surface, but decreases the amount of sputtered vapor that reaches a substrate directly in front of the target. The reduced deposition rate observed in the HiPIMS discharge is likely to be a combination of all the above-mentioned factors. To resolve this issue, modeling of the HiPIMS process is needed to explore the contribution from each of these factors. ${ }^{202}$

Among the early claims about the HiPIMS technique was improved target utilization. ${ }^{16}$ Indeed, Liebig et al. ${ }^{203}$ have shown using 2D OES that the sputter distribution of the target is wider for an HiPIMS discharge than for dcMS. Similar findings have been reported by Clarke et al. ${ }^{204}$ This is consistent with empirical observations that show that the width of the ion current density distribution in the target vicinity and thus the erosion width increases with increased target current and voltage, ${ }^{57}$ both of which are significantly higher for HiPIMS than for dcMS.

There have been some attempts to increase the deposition rate in the HiPIMS discharge. Konstantinidis et al. ${ }^{205}$ placed an inductive coil, to create an inductively coupled discharge, halfway between the target and the substrate in order to increase the conductivity of the interelectrode gas. They demonstrate increased ion collection at the substrate with increased $\mathrm{rf}$ power to the inductive coil and claim this effect could be used to minimize the decrease in deposition rate due to self-sputtering as it would make it easier for the metal ions to leave the magnetic trap. Also, Vlček et al. ${ }^{206}$ have demonstrated an increase in the deposition rate (up to 1.9 times) by increasing the target surface temperature (up to $1700^{\circ} \mathrm{C}$ ) for a titanium target. They point out that the target temperature can be controlled over a wide range in an HiPIMS operation. A more detailed study of the target melting, including the spatial and temporal variation of the target surface temperature for various discharge conditions, was reported by Tesař et al. ${ }^{207}$

\section{Self-sputtering}

During the pulse the sputtered atoms replace the working gas atoms in the vicinity of the target to some extent. These atoms eventually thermalize and in some cases they experience electron impact ionization. A fraction of those ions are attracted toward the target surface by the cathode fall. Thus, for high enough electron density a significant fraction of the target current is due to ions of the sputtered vapor that are attracted back to the target and participate in the sputtering process. This is referred to as self-sputtering. The sustainability of the self-sputtering process depends on various parameters such as the working gas, the sputter yield, the secondary electron emission yield, and the target voltage. It is also important to remember that self-sputtering has to release enough secondary electrons to maintain a high probability of ionizing those newly sputtered particles. Anders et al. ${ }^{117,197}$ argue that for the ion bombarding energies typical for an HiPIMS discharge the secondary electron emission is determined by the potential energy of the arriving ion projectile rather than its kinetic energy. For a potential emission to occur the potential energy (ionization potential) of the projectile has to exceed twice the work function of the target material. A fit to experimentally determined secondary electron emission yield for various ions on clean surfaces is given as ${ }^{208,209}$

$$
\gamma_{\mathrm{SE}}=0.032\left(0.78 \mathcal{E}_{\mathrm{iz}}-2 \phi\right),
$$

where $\mathcal{E}_{\mathrm{iz}}$ is the neutralization energy of the ion and $\phi$ is the work function of the target surface. The process can only occur if $0.78 \mathcal{E}_{\mathrm{iz}}>2 \phi$. It has been pointed out by Anders ${ }^{117,197}$ that the multiple charged metal ions are crucial for the transition of the discharge from noble gas ion sputtering to self-sputtering. He argues that singly charged metal ions (in ground state) cannot create the secondary electrons necessary to maintain metal self-sputtering. Thus, doubly or higher charged metal ions have to be present in the discharge in order for it to become self-sustained. The work function $\phi$ and the first and second ionization energies for several common elements are shown in Table II. We note that for some of the common metals like $\mathrm{Cr}$ and $\mathrm{Ti}$ the ionization energy to create doubly charged ions is relatively low, compared to the ionization energy of argon. Thus the concentration of the doubly charged ions of the sputtered vapor is expected to be high. In fact, Andersson and Anders ${ }^{210}$ have demonstrated a gasless self-sputtering from a high sputter yield copper target in an HiPIMS discharge. A vacuum-arc discharge was used to initiate the plasma at a background pressure of $10^{-3} \mathrm{~Pa}$. It has also been suggested to use a pulsed laser ablation to trigger the plasma and then run a gasless self-sputtering process. ${ }^{211}$ Furthermore, it has been demonstrated that the magnetron discharge not only self-sustains but amplifies via self-sputtering runaway to high discharge currents. ${ }^{17,197,210,212}$ Self-sputtering runaway is driven by a positive feedback, where a higher flux of ions leads to increased sputtering, which in turn leads to more neutral atoms that can be ionized in the vicinity of the target and more ions lead to increased sputtering. This continues as 
TABLE II. Work function $\phi$ and the first and second ionization energies for several common elements. ${ }^{\text {a }}$

\begin{tabular}{lccc}
\hline \hline Element & $\phi(\mathrm{eV})$ & $\mathcal{E}_{\mathrm{iz}, 1}$ & $\mathcal{E}_{\mathrm{iz}, 2}$ \\
\hline $\mathrm{Ar}$ & N/A & 15.76 & 27.63 \\
$\mathrm{Al}$ & $4.08-4.28$ & 5.99 & 18.83 \\
$\mathrm{Cu}$ & 4.9 & 7.73 & 20.29 \\
$\mathrm{Ti}$ & $4.1-4.3$ & 6.82 & 13.58 \\
$\mathrm{Cr}$ & 4.5 & 6.77 & 16.50 \\
\hline \hline
\end{tabular}

${ }^{\mathrm{a}}$ From Anders et al. (Ref. 117).

long as enough power is being supplied, e.g., if the power supply has a large capacitor that keeps the target voltage constant for the entire pulse length. Horwat and Anders ${ }^{213}$ have measured the IEDF during such self-sputtering from a copper target. Close to the target they identify the tail of the Thompson distribution that transforms to a shifted Maxwellian as the ions are accelerated and cooled. The selfsputtering can operate in a self-sustained mode, when the ions of the sputtered vapor are created at high enough rate that the ions of the working gas are not needed. The condition for sustained self-sputtering is expressed as ${ }^{18,211,214}$

$$
\Pi_{\mathrm{ss}}=\alpha \beta_{\mathrm{t}} Y_{\mathrm{ss}}=1 \text {, }
$$

where $\alpha$ is the probability of ionization of the sputtered atom, $\beta_{\mathrm{t}}$ is the probability that the newly formed ion of the sputtered vapor returns to the target, and $Y_{\mathrm{ss}}$ is the selfsputter yield of the ion. This is a steady state situation and the current remains constant. Note that since $\alpha<1$ and $\beta_{\mathrm{t}}<1$ the condition $Y_{\mathrm{ss}}>1$ is necessary but not sufficient for achieving sustained self-sputtering. The transient phase of self-sputtering runaway occurs when $\Pi_{\mathrm{ss}}>1$. This runaway occurs at a well-defined threshold power, determined by the discharge voltage and is readily obtained for high sputter yield materials such as copper, silver, and zinc. ${ }^{18}$ But, a runaway to a high density discharge has also been observed for transition metals ${ }^{18}$ and target materials of low sputter yield such as carbon and silicon, ${ }^{215}$ but in the presence of working gas. This observation has lead to a generalized runaway criterion,

$$
\Pi=\sum_{j} \alpha_{j} \beta_{j} Y_{j}>1,
$$

which involves both target and working gas atoms (ions), and $\alpha_{j}$ is the probability of ionization of atom $j, \beta_{j}$ is the return probability of ion $j$, and $Y_{j}$ is the sputter yield when ion $j$ bombards the target, which can be either an atom (ion) of the working gas or the sputtered vapor. Sputtering by the working gas ions here provides a supporting feed into the self-sputtering cycle, when in addition target atoms are sputtered by working gas impact. This allows runaway to occur at lower threshold voltages than for pure self-sputtering as the effective sputter yield is larger than the self-sputter yield. Thus, the ionization of the working gas atoms, their bombardment of the target, and their subsequent return to the ionization region results in a "gas recycling" runaway that can play a dominant role in the runaway for low sputter yield target materials. ${ }^{215}$ So runaway of the discharge current is due to a recycling mechanism, either by self-sputtering or by working gas recycling.

\section{E. Reactive sputtering}

Reactive sputtering, where metal targets are sputtered in a reactive gas atmosphere to deposit compound materials, is of utmost importance in various technologies, including transparent conductive oxides, permeation barrier coatings, hard coatings, etc. In reactive sputtering processes a reactive gas (e.g., $\mathrm{O}_{2}, \mathrm{~N}_{2}$, etc.) is mixed to the noble working gas to synthesize a compound film. The high electron density in the HiPIMS discharge is expected to enhance the dissociation of the molecular gas, which is sometimes considered beneficial for the oxided or nitride deposition. ${ }^{216}$ The presence of reactive gas can also lead to the formation of compound material on the target surface, often referred to as target coverage or target poisoning. ${ }^{217}$ Due to this target coverage the reactive sputtering process is inherently unstable, and is commonly represented in a familiar hysteresis curve that shows, e.g., the deposition rate or the target voltage versus the flow rate of the reactant molecular gas. ${ }^{218}$ The target voltage versus oxygen flow rate for a dcMS while sputtering a Ce target in an $\mathrm{Ar} / \mathrm{O}_{2}$ discharge is shown in Fig. 12. It can be seen that for an oxygen flow rate in the range $0.6-0.8 \mathrm{sccm}$ the discharge can be operated at three different target voltages. The upper curve refers to metal mode sputtering (where the target remains clean) and the lower curve to oxide mode sputtering (where the target is fully covered with a compound). Due to fast feedback it was possible to operate in the inherently unstable region in the middle (where the target is partially covered with a compound). The hysteresis effect originates from the changing target conditions due to the reaction of the target surface with the reactive gas. ${ }^{71}$ Sputtering at low reactive gas flows, where there is no significant reaction with the sputtering target, is referred to as metal mode sputtering, while for high flows of reactive gas, when a compound has formed on the target, it is referred to as the compound mode or the poisoned mode sputtering. The hysteresis occurs if the

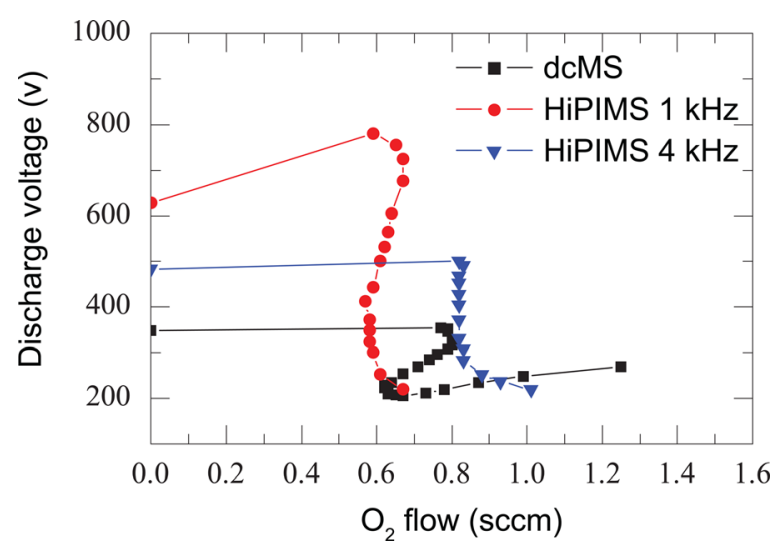

FIg. 12. (Color online) Discharge voltage $V_{\mathrm{d}}$ as a function of the $\mathrm{O}_{2}$ flow during reactive dcMS and HiPIMS of a Ce target. For the HiPIMS process pulsing frequencies of 1 and $4 \mathrm{kHz}$ are shown. The average discharge power was $70 \mathrm{~W}$ and the argon partial pressure was $0.65 \mathrm{~Pa}$ at a pumping speed of 251/s. After Aiempanakit et al. (Ref. 219). 
effective etching rate of the compound is lower than for the pure metal, which is commonly the case due to a lower sputter yield of the compound. Also, due to a change in the secondary electron emission yield as a compound is formed, more or less of the discharge current at the target surface can be carried by the electrons rather than the ions. ${ }^{71,220}$ This means that the current rises for some materials but decreases for others when sputtering in the compound (transition) mode.

The low deposition rate observed for the HiPIMS discharge and discussed in Sec. IIIC is of particular concern for reactive magnetron deposition of oxides due to the low sputter yield of most oxides. This concern has been confirmed by several reports on reactive sputtering with HiPIMS, a factor of 4-7 lower deposition rate for reactive sputtering of $\mathrm{TiO}_{2}$ from a Ti target, ${ }^{221}$ a factor of 3-4 lower for reactive sputtering of $\mathrm{AlO}_{x}$ from an $\mathrm{Al}$ target ${ }^{222}$ and up to a factor of 3 lower for reactive sputter deposition of $\mathrm{CrN}_{x}$ from a Cr target, ${ }^{223}$ compared to pulsed dcMS at the same average power.

For reactive sputtering it can be argued that higher deposition rates can be achieved if operated in the unstable region of the transition between the two target states. Growth of stoichiometric compound films at relatively high deposition rates can be achieved in the intermediate target coverage regime (or the transition zone) between the metallic and compound mode. This can be achieved by fast feedback control of the partial pressure of the reactive gas. Sproul et al. ${ }^{224}$ discuss a feedback system to control reactive sputtering by monitoring the partial pressure or mass flow of the reactive gas, the optical emission from the sputtered vapor, or the discharge voltage to control the partial pressure of the reactive gas. Feedback control of the reactive process has been demonstrated for HiPIMS by Audronis et al. ${ }^{225}$ using optical emission as input for the feedback control.

In the transition zone there is a balance between the removal and formation of the compound film on the target surface. The total number of sputtered compound molecules per unit time removed from the target is

$$
\frac{J_{\mathrm{i}} Y_{\mathrm{c}} \theta_{\mathrm{t}} S_{\mathrm{RT}}}{q},
$$

where $J_{\mathrm{i}}$ is the ion target current density, $Y_{\mathrm{c}}$ is the sputter yield of the compound, $q$ is the charge state of the incident ion, $\theta_{\mathrm{t}}$ is the fraction of the target covered by the compound, and $S_{\mathrm{RT}}$ is the race track area. ${ }^{218}$ Similarly the formation of the compound film on the target surface is described with

$$
n \Gamma \gamma_{\mathrm{t}}\left(1-\theta_{\mathrm{t}}\right) S_{\mathrm{RT}}
$$

where $\gamma_{t}$ is the sticking coefficient of the reactive molecule on the metallic portion of the target surface, $n$ is the number of reactive atoms in a molecule, and $\Gamma$ is the flux rate of molecules on the target surface which is proportional to the partial pressure of the reactive gas. ${ }^{217,218}$ In steady state, the formation of the compound and the removal of the compound film balance

$$
n \Gamma \gamma_{\mathrm{t}}\left(1-\theta_{\mathrm{t}}\right)=\frac{J_{\mathrm{i}} Y_{\mathrm{c}} \theta_{\mathrm{t}}}{q}
$$

The peak target current density in an HiPIMS discharge is very large (often $2-3$ orders of magnitude larger than in dcMS), which leads to a very high instantaneous erosion rate during the pulse-on time which may clean the target surface and displace the formation of compound film to higher reactive gas flow. Rarefaction in front of the target (discussed in Sec. IV B) also applies to the reactive gas and may lower the impingement rate of reactive gas atoms significantly and hamper the formation of compound film on the target surface. $^{35,42}$

Hysteresis-free reactive sputtering has been demonstrated in an HiPIMS discharge ${ }^{42}$ and allows for operation in the transition zone even without feedback control. Aiempanakit et al. ${ }^{219}$ demonstrate suppression or elimination of the hysteresis. This is shown in Fig. 12, where for a dcMS a relatively wide unstable region is observed while for an HiPIMS the width of the unstable region is substantially smaller and decreases with increasing repetition frequency. The reason for the reduction, or removal of the hysteresis in some cases, for HiPIMS is not fully understood. Kubart et al. ${ }^{226}$ also explored the hysteresis effect in am HiPIMS discharge in $\mathrm{Ar} / \mathrm{O}_{2}$ mixtures using a Ti target. For fixed pulse length and a constant average power, they find an optimum frequency minimizing the hysteresis. They explain their results by an effect of gas rarefaction and gas refill time. For off times longer than the refill time (lower frequency), the effective oxygen pressure increases. For higher pulsing frequency (assuming constant average power), the extent of gas density reduction is lower due to the lower peak current and target poisoning is more pronounced again. Furthermore, it has been shown that increasing the repetition frequency while keeping the average power fixed leads to increased deposition rate for reactive sputtering of $\mathrm{TiO}_{2}$ in $\mathrm{Ar} / \mathrm{O}_{2}$ discharge and repetition frequency of $4 \mathrm{kHz}$ gives almost the same deposition rate as dcMS. ${ }^{181}$

It has recently been observed that the current waveform in the reactive $\mathrm{Ar} / \mathrm{N}_{2}$ HiPIMS discharge when sputtering Ti target is highly dependent on the pulse repetition frequency, unlike the nonreactive Ar discharge. ${ }^{227}$ The current is found to increase significantly as the frequency is lowered. This has been attributed to an increase in the secondary electron emission yield for self-sputtering when the nitride forms on the target at low frequencies. The secondary electron emission yield is higher for a nitrided (poisoned) Ti target than a clean Ti target when self-sputtering is the dominant sputtering mechanism, since as discussed in Sec. III D the electron emission yield for self-sputtering by singly ionized metal is essentially zero, which is not the case for $\mathrm{N}^{+}$. This differentiates HiPIMS from dcMS where self-sputtering is not as important. The current rises sharply as the pulse repetition frequency is lowered as a result of increased target nitridation. However, Greczynski et al. ${ }^{223}$ report on a decrease in deposition rate with increasing $n_{\mathrm{N}}^{2} / n_{\mathrm{Ar}}$ ratio. They relate this decrease to nitride formation on the surface of the sputtering target (poisoning effect) and lower sputtering efficiency of 
the $\mathrm{N}_{2}$ gas compared to argon gas. They also point out that the relative drop in the deposition rate with increasing $n_{\mathrm{N}}^{2} / n_{\mathrm{Ar}}$ ratio is similar for dcMS and HiPIMS.

\section{PHYSICS OF HIPIMS}

The conventional magnetron sputtering discharge has been successfully modeled by a 2D fluid model of the dc discharge $^{228,229}$ and by self-consistent particle-in-cell (PIC)/ Monte Carlo collisional simulations of the dc (Refs. 230-234) and the rf (Refs. 235 and 236) discharges. However, for the HiPIMS discharge the situation is more complicated due to the high density of the charged species and the long time scales required to resolve the full pulse period. Thus other approaches have to be taken in order to improve our understanding of the processes dictating the HiPIMS discharge. There has been a significant advancement in the understanding of the physics of the HiPIMS discharge over the past few years and various approaches have been used to model the discharge. Here we review some of the models that have been developed and summarize the current understanding of the mechanisms involved.

\section{A. Deposition rate: A phenomenological model}

Christie $^{195}$ developed a phenomenological model to determine the ionized fraction of the target material arriving at the substrate and to explain the low deposition rate observed in HiPIMS discharges. This model is referred to as the target material pathway model. The model was later modified by Vlček et al., ${ }^{237}$ Vlček and Burcalová, ${ }^{238}$ and Lundin. ${ }^{239}$ Vlček and Burcalová ${ }^{238}$ added the feature of evaluating the fraction of the sputtered vapor returning to the target. Thus, this model allows for the calculation of the fraction of ionized sputtered vapor that turns back to the target, the degree of ionization of the sputtered vapor, and the ionized flux fraction onto the substrate. Figure 13 is a schematic of the phenomenological model that shows the collective particle dynamics, where the Roman numerals correspond to the different processes taking place (described in the following). Here we follow the discussion by Lun$\operatorname{din}^{239}$ There are two types of ions that are accelerated across the high voltage sheath to bombard the cathode target surface, the ions of the noble working gas $G^{+}$(process I), and the ions of the sputtered vapor $M^{+}$(process II). These two types of ions will sputter away different amounts of target material depending on their respective sputter yield (III). After the sputtering event the sputtered vapor is transported out into the plasma. It is assumed that the mean free path for elastic collisions against the working gas is so long that a negligible fraction of the neutral sputtered species returns to the target. On their way to the substrate there is a probability $\alpha$ of an ionizing event (IV), while they are in the ionization region (IR) next to the target surface through, for example, a collision with an electron. A fraction of those ions (denoted by $\beta_{\mathrm{t}}, 0 \leq \beta_{\mathrm{t}} \leq 1$ ) will be close enough to the cathode fall and have a low enough kinetic energy to be back-attracted to the target surface (V) and take part in the sputtering. The rest of the ions will escape into the bulk plasma (VI) [with probabil-

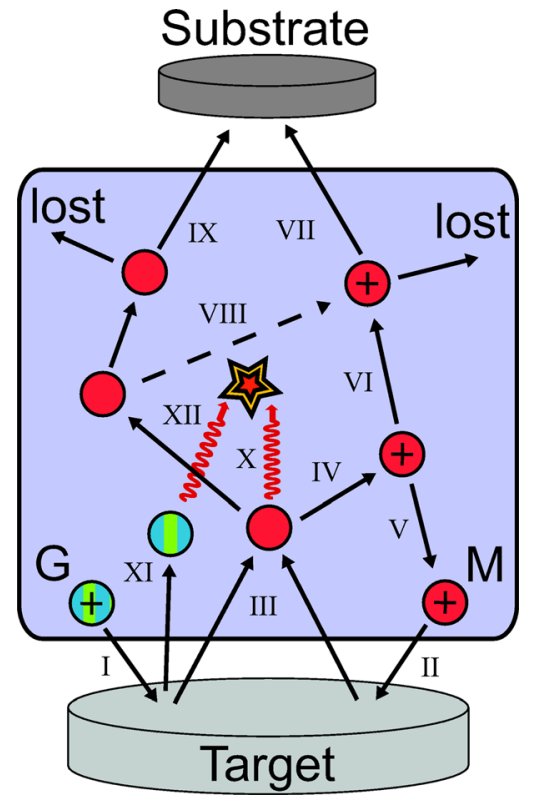

FIG. 13. (Color online) Schematic of the target material pathway model. The letters $G$ and $M$ stand for gas and metal ions, respectively. The lower half of the discharge volume represents the ionization region and the upper half the bulk plasma (BP) (see Fig. 16 for definitions). Processes VI and XII represent the flow of sputtered flux from the former to the latter. From Lundin (Ref. 239).

ity $\left(1-\beta_{\mathrm{t}}\right)$ ], where they either will be lost to the walls [with probability $\left.\left(1-\xi_{\mathrm{i}}\right)\right]$ or end up at the substrate position (VII) (with probability $\xi_{\mathrm{i}}$ ). The neutrals of the sputtered vapor that have not undergone an ionizing collision in the IR might later get ionized in the bulk of the plasma (VIII) (with probability $\gamma$ ) due to enhanced electron confinement, while being too far away to be affected by the negative potential drop. They may also be lost to the chamber walls [probability $\left.\left(1-\xi_{\mathrm{n}}\right)\right]$, or arrive at the substrate to take part in the film growth (IX) (probability $\xi_{\mathrm{n}}$ ). The parameters $\xi_{\mathrm{n}}$ and $\xi_{\mathrm{i}}$ take into account the geometry of the system, but the latter can also be affected by the magnetic field configuration. ${ }^{39}$ During the transport there is also a likelihood that metal neutrals will collide with the neutral gas background (X). These collisions lead to heating of the gas followed by expansion (decrease in gas density in front of the target), in a process that is referred to as gas rarefaction, which will be further discussed in Sec. IV B. A few percent of the bombarding gas ions will not participate in sputtering, but merely be neutralized at the target surface and reflected back to the plasma (XI). These reflected working gas atoms will have an inherently high energy, since they were first accelerated toward the cathode (as ions) by the high potential cathode fall and much of that energy was not transferred to the target atoms. ${ }^{240} \mathrm{~A}$ fraction of the reflected gas neutrals will also collide with the neutral background (XII) and thereby enhance the gas rarefaction, while others will quickly leave the bulk plasma region.

The total total number of atoms of the sputtered vapor from the target is then

$$
M_{\text {total }}=G_{\text {target }}^{+} Y_{\text {tg }}+M_{\text {target }}^{+} Y_{\text {ss }},
$$


where $G_{\text {target }}^{+}$is the number of ions of the working gas incident on the target, $M_{\text {target }}^{+}$is the number of the ions of the sputtered vapor incident on the target, $Y_{\mathrm{tg}}$ is the sputter yield for ions of the working gas hitting the target, and $Y_{\mathrm{ss}}$ is the self-sputter yield for the ions of the target material hitting the target. The sputtered target material is then transported out into the plasma. Some of these neutrals will be ionized in the target vicinity mainly due to electron impact ionization. The fraction of ions of the sputtered vapor in the total ion flux onto the target is ${ }^{238}$

$$
m_{\text {target }}=\frac{M_{\text {target }}^{+}}{M_{\text {target }}^{+}+G_{\text {target }}^{+}},
$$

which is in the range $0 \leq m_{\text {target }} \leq 1$, so

$$
M_{\text {total }}=Y_{\text {tg }}\left(1-m_{\text {target }}\right)+Y_{\text {ss }} m_{\text {target }}\left(G_{\text {target }}^{+}+M_{\text {target }}^{+}\right)
$$

and the number of ions of the sputtered vapor returning to the target is

$$
M_{\text {target }}^{+}=M_{\text {total }} \alpha \beta_{\mathrm{t}} .
$$

Combining Eqs. (21) and (24) gives the total number of metal atoms sputtered per gas ion incident on the target,

$$
M_{\text {total }}=G_{\text {target }}^{+} \frac{Y_{\mathrm{tg}}}{1-\alpha \beta_{\mathrm{t}} Y_{\mathrm{ss}}} .
$$

The number of neutrals of the sputtered vapor incident on the substrate per noble gas ion incident on the target is given as

$$
M_{\text {substrate }}=G_{\text {target }}^{+} \frac{Y_{\mathrm{tg}}(1-\alpha) \beta_{\mathrm{t}} \xi_{\mathrm{n}}(1-\gamma)}{1-\alpha \beta_{\mathrm{t}} Y_{\mathrm{ss}}} .
$$

A fraction of the ions of the sputtered vapor will be created close enough to the cathode fall to be back-attracted to the target surface and take part in the sputtering. The number of target material ions incident on the target per gas ion incident on the target

$$
M_{\text {target }}^{+}=G_{\text {target }}^{+} \frac{Y_{\mathrm{tg}} \alpha \beta_{\mathrm{t}}}{1-\alpha \beta_{\mathrm{t}} Y_{\mathrm{ss}}} .
$$

The rest of the ions with probability $\left(1-\beta_{\mathrm{t}}\right)$ will reach the bulk plasma where they either will be lost to the chamber walls with probability $\left(1-\beta_{\mathrm{t}}\right)\left(1-\xi_{\mathrm{i}}\right)$ or end up at the substrate position taking part in the film growth. Thus the number of target material ions incident on the substrate per gas ion incident on the target is

$$
M_{\text {substrate }}^{+}=G_{\text {target }}^{+} \frac{Y_{\mathrm{tg}}\left[\alpha\left(1-\beta_{\mathrm{t}}\right) \xi_{\mathrm{i}}+(1-\alpha) \gamma \xi_{\mathrm{i}}\right]}{1-\alpha \beta_{\mathrm{t}} Y_{\mathrm{ss}}} .
$$

From the above-presented equations we can derive equations for the ionized flux fraction of target material that reaches the substrate,

$$
\theta=\frac{\left[\alpha\left(1-\beta_{\mathrm{t}}\right)+\gamma(1-\alpha)\right] \xi_{\mathrm{i}} / \xi_{\mathrm{n}}}{\left[\alpha\left(1-\beta_{\mathrm{t}}\right)+\gamma(1-\alpha)\right] \xi_{\mathrm{i}} / \xi_{\mathrm{n}}+(1-\alpha)(1-\gamma)},
$$

which is independent of the sputter yield, and the fraction of ions impacting the target which are ions of the sputtered vapor is

$$
\zeta=\frac{\alpha \beta_{\mathrm{t}} Y_{\mathrm{tg}}}{1+\alpha \beta_{\mathrm{t}}\left(Y_{\mathrm{tg}}-Y_{\mathrm{ss}}\right)} .
$$

Note that when $Y_{\text {tg }} \approx Y_{\text {ss }}$,

$$
\zeta=\alpha \beta_{\mathrm{t}} Y_{\mathrm{tg}} \leq Y_{\mathrm{tg}}
$$

Furthermore, Christie ${ }^{195}$ introduced a normalized deposition rate coefficient,

$$
\begin{aligned}
\psi\left(V_{\mathrm{d}}\right) & =\frac{\rho_{\text {HiPIMS }}\left(V_{\mathrm{d}}\right)}{\rho_{\mathrm{dc}}\left(V_{\mathrm{d}}, \alpha=0\right)} \\
& =\frac{(1-\alpha)\left(1-\gamma+\gamma \xi_{\mathrm{i}} / \xi_{\mathrm{n}}\right)+\alpha\left(1-\beta_{\mathrm{t}}\right) \xi_{\mathrm{i}} / \xi_{\mathrm{n}}}{1+\alpha \beta_{\mathrm{t}}\left(Y_{\mathrm{tg}}-Y_{\mathrm{ss}}\right)}
\end{aligned}
$$

where $\rho_{\mathrm{dc}}=\xi_{\mathrm{n}} Y_{\mathrm{tg}}$ is the deposition rate for conventional dcMS and $\rho_{\text {HiPIMS }}$ is the deposition rate for HiPIMS discharge and has contribution from both neutrals and ions of the sputtered vapor due to sputtering by both ions of the working gas and the ions of the sputtered vapor. Note that if $\beta_{\mathrm{t}} \approx 1, \xi_{\mathrm{i}}=\xi_{\mathrm{n}}$, and $Y_{\mathrm{tg}} \approx Y_{\mathrm{ss}}$ we find

$$
\psi \approx 1-\alpha
$$

which implies that as more of the sputtered vapor is ionized the rate will decrease, approaching zero as the ionization fraction approaches unity. This is a natural consequence of the primary assumption $\beta_{\mathrm{t}} \approx 1$, i.e., all the ions of the sputtered vapor are drawn back to the target.

To demonstrate the dependence of the above-mentioned parameters for a typical metal target we use $\beta_{\mathrm{t} \text {,initial }}=0.8$ as a starting value and allow for the ionization fraction $\alpha$ to increase from 0 to 1 . In addition, when the presence of process gas is assumed and we impose the condition

$$
\alpha \beta_{\mathrm{t}} Y_{\mathrm{ss}}<1
$$

to ensure that the system is in equilibrium and the number of atoms of the sputtered vapor is not increasing. That is, we do not have sustained-self-sputtering. We follow Christie ${ }^{195}$ and for computational reasons write $\beta_{\mathrm{t}}=0.99 /\left(\alpha Y_{\mathrm{ss}}\right)$. This condition implies that $99 \%$ of the total number of the sputtered atoms are sputtered by ions of the sputtered vapor. As the ionization fraction $\alpha$ increases the value of $\beta_{\mathrm{t}}$ decreases, which implies that a smaller fraction of the ions of the sputtered vapor is trapped by the magnetic field. If the losses of ions and neutrals of the sputtered vapor are the same as they flow toward the substrate we can write $\xi_{\mathrm{i}}=\xi_{\mathrm{n}}$. The sputter yield increases with applied target voltage. Anders ${ }^{196}$ gives the sputter yield for various materials as a fit of the form

$$
Y_{k}=a_{k} \mathcal{E}_{k}^{b_{k}}
$$

where the parameters $a_{k}$ and $b_{k}$ are given in Table III. Figure 14 shows the dependence of the normalized deposition rate coefficient $\psi$, the fraction of ions impacting the target which 
TABLE III. Fitting parameters for the sputter yield for aluminum and copper. $^{\text {a }}$

\begin{tabular}{lcc}
\hline \hline & $a_{\mathrm{k}}$ & $\mathrm{b}_{\mathrm{k}}$ \\
\hline $\mathrm{Ar}^{+} \rightarrow \mathrm{Al}$ & 0.0296 & 0.512 \\
$\mathrm{Al}^{+} \rightarrow \mathrm{Al}$ & 0.1042 & 0.370 \\
$\mathrm{Ar}^{+} \rightarrow \mathrm{Cu}$ & 0.1421 & 0.468 \\
$\mathrm{Cu}^{+} \rightarrow \mathrm{Cu}$ & 0.0691 & 0.556 \\
\hline \hline
\end{tabular}

${ }^{\mathrm{a}}$ From Anders (Ref. 196).

are ions of the sputtered vapor $\zeta$, the ionized flux fraction of target material that reaches the substrate $\theta$, and the fraction of ions of the sputtered vapor directed back to the target $\beta_{\mathrm{t}}$ as a function of the degree of ionization of the sputtered vapor $\alpha$ for aluminum and copper targets. The ion bombarding energy is assumed to be $560 \mathrm{~V}, \gamma=0.1$ and $\xi_{\mathrm{i}} / \xi_{\mathrm{n}}=0.75$. Keep in mind that $\alpha$ is expected to increase with increasing power density. We see in Fig. 14 that as the degree of ionization of the sputtered vapor increases the normalized deposition rate coefficient $\psi$ decreases and the fraction of ions impacting the target which are ions of the sputtered vapor increases. Thus the model predicts that the lower deposition rate is due to significant backscattering of metal ions back to
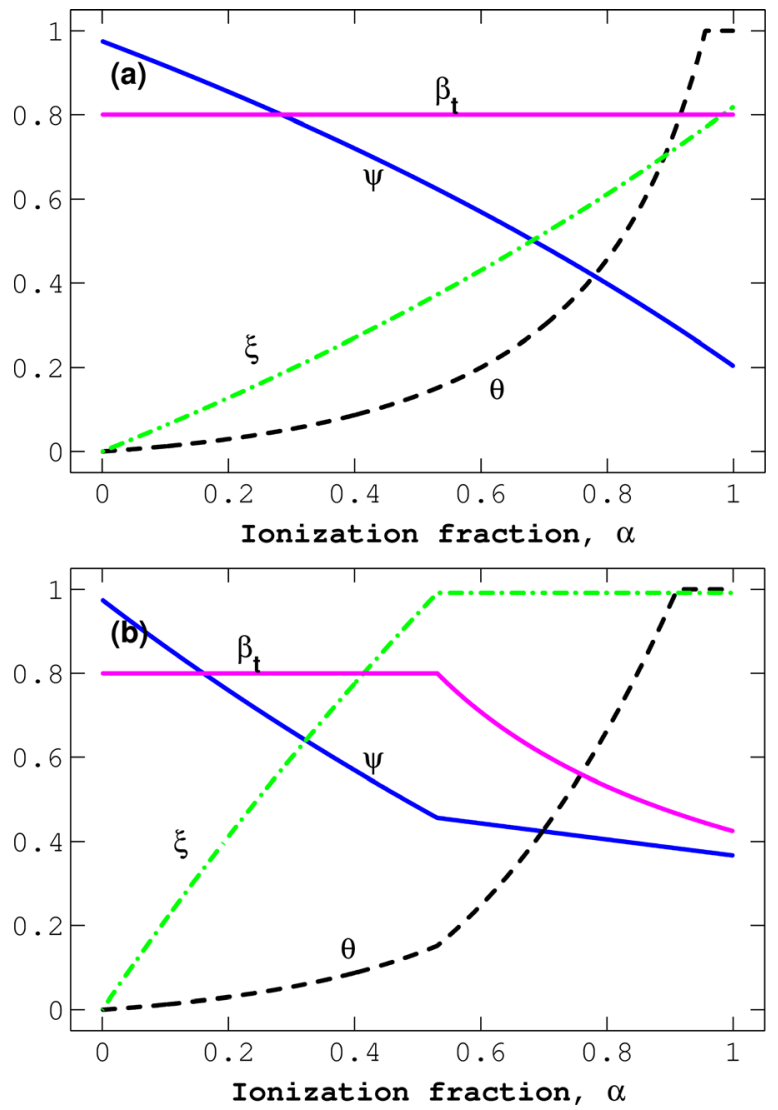

FIG. 14. (Color online) Calculated values of the normalized deposition rate coefficient $\psi$, the fraction of ions impacting the target which are ions of the sputtered vapor $\zeta$, the ionized flux fraction of target material that reaches the substrate $\theta$, and the fraction of ions of the sputtered vapor directed back to the target $\beta_{\mathrm{t}}$, as a function of the degree of ionization of the sputtered vapor for (a) aluminum target and (b) copper target. the target. We also note that for copper target [Fig. 14(b)] with $\alpha>\sim 0.5$ only copper ions are sputtering the target and for $\alpha>\sim 0.9$ only copper ions reach the substrate. It should be noted that this is a steady-state model that does not account for time-dependent processes such as self-sputtering runaway. It should also be noted that this calculation depends on the assumed initial return probability, here $\beta_{\mathrm{t}, \text { initial }}=0.8$, and on the constraint of Eq. (34). The model demonstrates the central importance of the ionization degree $\alpha$ and the return probability $\beta_{\mathrm{t}}$, while the determination of their actual values in a specific discharge must rely on experimental data (Sec. III) and/or more advanced analysis and modeling (Secs. IV C and IV D, to follow).

Vlček and Burcalová ${ }^{238}$ improved the model further by including the effects of the secondary electron emission, the sputter yield, and the effective energy lost per electron-ion pair created when calculating the fraction of ionized sputtered vapor that is directed back to the target $\beta_{\mathrm{t}}$. Substituting Eq. (24) into Eq. (23) gives

$$
\alpha=\frac{m_{\text {target }}}{\beta_{\mathrm{t}}} \frac{1}{Y_{\mathrm{tg}}+m_{\mathrm{target}}\left(Y_{\mathrm{ss}}+Y_{\mathrm{tg}}\right)} .
$$

This can be rewritten as

$$
m_{\text {target }}=\alpha \beta_{\mathrm{t}} Y_{\mathrm{t}} \leq Y_{\mathrm{t}}
$$

where the effective sputter yield of the target is

$$
Y_{\mathrm{t}}=Y_{\mathrm{tg}}\left(1-m_{\text {target }}\right)+Y_{\mathrm{ss}} m_{\text {target }} .
$$

The fraction of ionized sputtered atoms directed back to the target $\beta_{\mathrm{t}}$ is assumed to be the same as the fraction of ionized working gas atoms $\beta_{\mathrm{g}}$. If we assume all the applied target voltage drops across the cathode sheath we get

$$
\gamma_{\mathrm{SE}, \mathrm{eff}}\left(1-d_{\mathrm{c}}\right) \beta_{\mathrm{t}} \frac{V_{\mathrm{d}}}{\mathcal{E}_{\mathrm{c}, \mathrm{eff}}}=1,
$$

where $\gamma_{\text {SE,eff }}$ is the effective secondary electron emission coefficient of the target material and $\mathcal{E}_{\mathrm{c} \text {,eff }}$ is the effective energy loss per electron-ion pair produced, and $d_{\mathrm{c}}$ is the fraction of secondary electrons lost by diffusive transport to the substrate and to the chamber walls. Also,

$$
\gamma_{\mathrm{SE}, \mathrm{eff}}=(1-r)\left[\gamma_{\mathrm{mg}}\left(1-m_{\mathrm{target}}\right)+\gamma_{\mathrm{ss}} m_{\text {target }}\right],
$$

where $r$ is the recapture probability reducing the total secondary electron emission coefficient. The effective energy lost per electron-ion pair created is written as

$$
\mathcal{E}_{\mathrm{c}, \mathrm{eff}}=\mathcal{E}_{\mathrm{c}, \mathrm{g}}\left(1-m_{\text {target }}\right)+\mathcal{E}_{\mathrm{c}, \mathrm{t}} m_{\text {target }},
$$

where $\mathcal{E}_{\mathrm{c}, \mathrm{g}}$ and $\mathcal{E}_{\mathrm{c}, \mathrm{t}}$ are the energy lost per electron-ion pair created for the noble working gas and the sputtered vapor, respectively. Substituting Eqs. (40) and (41) into Eq. (39) gives

$$
\beta_{\mathrm{t}}=\frac{\mathcal{E}_{\mathrm{c}, \mathrm{g}}\left(1-m_{\mathrm{target}}\right)+\mathcal{E}_{\mathrm{c}, \mathrm{t}} m_{\mathrm{target}}}{V_{\mathrm{d}}\left(1-d_{\mathrm{c}}\right)(1-r)\left[\gamma_{\mathrm{mg}}\left(1-m_{\mathrm{target}}\right)+\gamma_{\mathrm{ss}} m_{\text {target }}\right]} .
$$


This model allows for calculation of $\alpha$ and $\beta_{\mathrm{t}}$ as a function of the applied target voltage $V_{\mathrm{d}}$ and the fraction of ions in the sputtered vapor directed onto the substrate. Also, simple scaling laws are achieved $\beta_{\mathrm{t}} \propto 1 / V_{\mathrm{d}}$ and if we assume $Y_{\mathrm{t}} \propto V_{\mathrm{d}}^{1 / 2}$ it is apparent that $\alpha \propto V_{\mathrm{d}}^{1 / 2}$ and $\alpha \beta_{\mathrm{t}} \propto V_{\mathrm{d}}^{-1 / 2}$.

\section{B. Neutral particle flow-rarefaction}

It is well known that for a conventional dcMS discharge collisions between the sputtered particles and the neutral working gas atoms results in gas heating and rarefaction. ${ }^{241,242}$ The sputtered atoms leaving the target are expected to have kinetic energy of a few $\mathrm{eV} .{ }^{243}$ The density of the sputtered vapor is significantly higher in an HiPIMS discharge than a dcMS. The energy transfer from the increasing number of sputtered atoms to the working gas atoms leads to heating of the working gas followed by expansion, a process that is referred to as rarefaction. Sometimes the term "sputtering wind" is used to describe this momentum transfer and displacement of the working gas by the sputtered vapor. ${ }^{244}$ The sputtering wind has a direction away from the target. This lowered density of the working gas leads to a reduction in the number of ions available for sputtering, which again leads to a reduction in sputter rate and thus deposition rate. The number of sputtered atoms in the vicinity of the target increases with increasing discharge current. This has been demonstrated by laser absorption spectroscopy of aluminum atoms comparing dcMS and a pulsed magnetron sputtering discharge at the lower end of the HiPIMS regime. ${ }^{245}$ There is almost an order of magnitude increase in the instantaneous density of aluminum atoms when pulsing the discharge at the same average power. Since the target current is much higher for an HiPIMS discharge than for a dcMS discharge the rarefaction effect is expected to be much more pronounced. This phenomena has been demonstrated experimentally for an HiPIMS discharge by Alami et al. ${ }^{199}$ and Vlček et al ${ }^{173,246}$ Using optical emission spectrometry Vlček et al. ${ }^{173,246}$ see almost an order of magnitude decrease in the density of atomic argon but slightly less dramatic decrease in the density of argon ions roughly 50-70 $\mu$ s into a $200 \mu$ s long pulse while sputtering $\mathrm{Cu}$ target. Liebig et al. $^{203}$ apply 2D OES to explore the spatial and temporal behavior of the argon working gas and also observe gas rarefaction. In addition, Vitelaru et $a l^{247}$ have recently performed time resolved tunable diode-laser absorption spectroscopy measurements of the argon metastable $\left(\mathrm{Ar}^{\mathrm{m}}\right)$ density in an HiPIMS discharge driven by $200 \mu$ s pulses while sputtering a Ti target. From the Doppler profile the evolution of the temperature and density were derived during the pulse as well as during the plasma afterglow. Typical results are shown in Fig. 15, where it is shown that the $\mathrm{Ar}^{\mathrm{m}}$ density sharply increases within the first $25 \mu \mathrm{s}$, followed by a severe $\mathrm{Ar}^{\mathrm{m}}$ depletion along with increasing gas temperature around the peak of the HiPIMS discharge current at about $80 \mu$ s into the pulse. The authors claim that the reduction of $\mathrm{Ar}^{\mathrm{m}}$ density is due to a strong depletion of neutral $\mathrm{Ar}$, which is likely an effect of gas rarefaction as well as due to electron impact ionization of Ar owing to the high plasma density

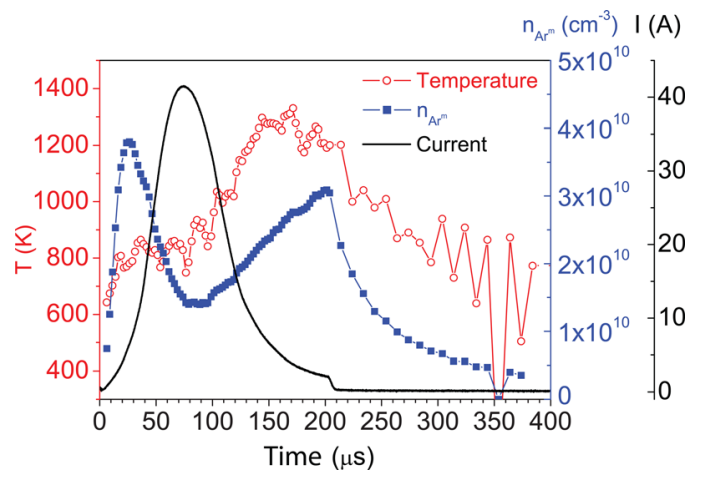

FIG. 15. (Color online) Typical results of time resolved time resolved tunable diode-laser absorption spectroscopy in HiPIMS taken at $1 \mathrm{~cm}$ from the target surface at an Ar gas pressure of 1.3 Pa. The full black curve displays the HiPIMS discharge current $I_{\mathrm{d}}(t)$ (corresponding to a peak current density of $0.5 \mathrm{~A} \mathrm{~cm}^{-2}$ ), the red curve with circles is the temperature of the $\mathrm{Ar}^{\mathrm{m}}$ and the blue curve with squares represents the $\mathrm{Ar}^{\mathrm{m}}$ density. Adapted from Vitelaru et al. (Ref. 247).

during the peak of the HiPIMS pulse. As the discharge current starts to decrease (decreasing plasma density) a strong increase of the $\mathrm{Ar}^{\mathrm{m}}$ density is again detected until the pulse is switched off at $200 \mu \mathrm{s}$, followed by the plasma afterglow characterized by a decay of both density and temperature of the $\mathrm{Ar}^{\mathrm{m}}$ as the plasma species are lost through recombination and diffusion toward the chamber wall. Hala et al. ${ }^{136}$ demonstrate the development of a dense plasma of the sputtered vapor close to the target and its expansion into the reactor. As the target atoms are sputtered off the target a density gradient is created that drives diffusion of both sputtered neutrals and ions away from the target. The wavefront of this expanding plasma propagates outward from the target along the axis of the magnetron discharge. In a pure argon

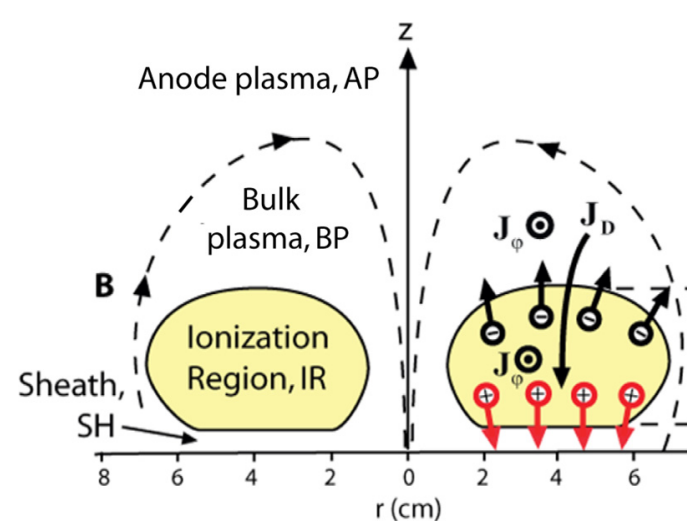

FIG. 16. (Color online) Left side shows the division of the plasma into different regions, adapted from Brenning et al. (Ref. 202). The ionization region is defined as the volume in which most of the ionization is located. It is a subvolume of the magnetic trap, the region with closed magnetic field lines (those that in both directions intersect the target). Recent optical studies (Ref. 136) show that during the first few tens of microseconds of an HiPIMS pulse the IR rapidly expands, and then stabilizes approximately coinciding with the magnetic field lines that intersect the active (race track) part of the target. The bulk plasma consists of the rest of the magnetic trap. The anode plasma (AP), finally, is the region with open magnetic field lines with at least one end intersecting the substrate, the anode, or the walls. The right side shows the currents carried by electrons and ions in, and across the borders of the different regions. 
discharge the propagating plasma has a hemispherical shape. In a reactive gas the plasma wavefront starts out hemispherical but changes to conical and then to droplike shape. This dense plasma travels at speeds in the range of $0.7-3.5 \mathrm{~km} / \mathrm{s}$ depending on the working gas composition and the gas pressure. ${ }^{136} \mathrm{~A}$ gas shock wave precedes this expanding dense plasma; thus confirming the existence of the ion-acoustic solitary wave expanding away from the cathode target. ${ }^{149}$ Hála et al. ${ }^{136,248}$ also report on emission from the working gas due to excitation by fast electrons during the ignition phase that spread outwards from the target at rather high speed $\left(24 \mathrm{~km} / \mathrm{s}\right.$ at $1.3 \mathrm{~Pa} \mathrm{Ar}$ and at $7.5 \mathrm{~km} / \mathrm{s}$ in $1.3 \mathrm{~Pa}$ of $\left.\mathrm{N}_{2}\right)$.

The behavior of the neutral particles during HiPIMS pulse was studied by a direct Monte Carlo method by Kadlec. ${ }^{249}$ $\mathrm{He}$ assumes argon discharge and titanium target. When the target current reaches the kA range very strong gas rarefaction is observed and the volume density of sputtered metal exceeds the working gas density several times. The gas starts to move rapidly away from the target as a shock wave a few microseconds into the pulse, similar to the shock wave measured by Hála et al. ${ }^{136,248}$ For a $200 \mu$ s long pulse ( $1 \mathrm{kA}$ and $1 \mathrm{kV}$ ) the argon density falls roughly an order of magnitude in front of the race track $100 \mu$ s into the pulse and the [Ti]/ [Ar] ratio reaches a value of roughly 5 . This shows that gas rarefaction is very strong in the HiPIMS discharge, indeed.

The reduction of gas density would not be much of a problem if the refill process were fast enough. Unfortunatel,y this does not seem to be the case in HiPIMS discharges ${ }^{193}$ due to high peak power densities. ${ }^{249}$ The effect of the gas refill was studied for different pulse off times by Lundin et al., ${ }^{193}$ which showed that the high peak currents commonly found in HiPIMS could not be attained when decreasing the off time below approximately $1 \mathrm{~ms}$. For pulse off times greater than $3 \mathrm{~ms}$ the influx of neutral gas could diffuse into the most intense area of the discharge and thereby provide enough source particles for ionization in order to recover the high peak currents. In a series of experiments with long pulses with constant voltage Lundin et al ${ }^{193}$ found a transition from an initial high current peak to a lower current steady plateau value. They proposed that the time duration of the peak was mainly determined by a new mechanism they proposed for gas rarefaction in the high momentary current densities in HiPIMS. The idea is that the integrated current, from the pulse start, corresponds to a number of atoms in the ionization region that have been ionized and attracted to the target given by

$$
\Delta n_{\mathrm{Ar}, \mathrm{iz}}=\frac{I_{\mathrm{d}}(t)}{e\left(1+\gamma_{\mathrm{SE}}\right)} d t
$$

If these ions, after recombination, return with so high speed that they spend negligible time in the ionization region, they can be regarded as practically lost. An approximate rarefaction time scale for this process, $\tau_{\text {raref,iz }}$, is the time it takes from the pulse start to ionize all argon atoms that are originally in the ionization region, $n_{\mathrm{Ar}, 0} V_{\mathrm{IR}}$, where $V_{\mathrm{IR}}$ is the volume of the ionization region. The scaling found by Lundin et al., ${ }^{193}$ both with discharge current and with gas pressure, indicated that this should be regarded as a major candidate for determining the time duration of the initial high current peak. It is not an important mechanism in dcMS discharges, since in these $\tau_{\text {raref,iz }}$ is longer than the refill time at the gas acoustic speed. This mechanism was not included in the simulations of Kadlec. ${ }^{249}$

\section{Ionization of the sputtered vapor}

The ionization of the sputtered vapor in low density $\left(n_{\mathrm{e}} \sim 10^{16} \mathrm{~m}^{-3}\right)$ sputtering devices, such as rf sputtering discharges ${ }^{250,251}$ and dcMS, ${ }^{66}$ is primarily due to the Penning process (e.g., for an argon discharge $\mathrm{Ar}^{\mathrm{m}}+\mathrm{M} \rightarrow \mathrm{Ar}+\mathrm{M}^{+}+$ e) and electron impact ionization is not very important. It was shown by Hopwood et al.,91,252 using a volume averaged global model of an ICP-MS, that for electron densities above $\sim 10^{17} \mathrm{~m}^{-3}$ electron impact ionization becomes the dominant ionization mechanism for the sputtered vapor while for electron densities below $\sim 10^{17} \mathrm{~m}^{-3}$ the Penning process is dominating. To explore the ionization processes in the HiPIMS discharge, the time dependent global (volume averaged) model developed by Ashida et al. ${ }^{253}$ and the above-mentioned (volume averaged) global model of the IPVD process in the ICP-MS discharge developed by Hopwood et al.,91,252 was combined and modified to describe the HiPIMS process. ${ }^{254}$ The results from this model indicate that electron impact ionization of the sputtered vapor is the dominant contributor to the high ionization fraction in the HiPIMS discharge. However, this model did not take into account the spatial variation of the density within the discharge so it was later improved by defining an IR next to the race track instead of taking a volume average over the entire chamber. ${ }^{255,256}$ This is referred to as ionization region model (IRM). The IRM is based on the fact that in the magnetron sputtering discharge a high density brightly glowing torus shaped plasma hovers in front of the cathode target extending a few centimeters, and is embedded in a lower density plasma bulk (see Fig. 16). The IRM is a model of the plasma chemistry in this high density plasma region, while the surrounding plasma can be treated by a bulk plasma model. ${ }^{257}$ The IRM is intended to use data from a real discharge as input [geometry, target and gas species, pressure, pulse shapes $I_{\mathrm{d}}(t)$ and $V_{\mathrm{d}}(t)$, etc.]. However, like in the target pathway model (discussed in Sec. IV A), the ion return probability $\beta_{\mathrm{t}}$ has to be assumed, measured, or separately motivated. The output is the plasma parameters, the compositions and degrees of ionization of all species, and the fluxes to the bulk plasma and to the target. Also, the fraction of the electric power that goes into heating the electrons $F_{\mathrm{PWR}}$, is an output variable, obtained through an energy self-consistency condition. Values in the range $0.1<F_{\mathrm{PWR}}<0.3$ are typically found when high current $\left(I_{\mathrm{d}, \max } \sim 100 \mathrm{~A}\right)$ pulses are modeled. The temporal variation is defined by a set of ordinary differential equations giving the first time derivatives of the electron energy and the particle densities for all the particles. The electron density is directly given by the ion densities assuming quasi-neutrality of the plasma. The treatment is essentially zero-dimensional, i.e., only volume averaged values over the whole ionization region volume for the electron, ion, and 
neutral densities are calculated. However, geometrical effects are included indirectly in the treatment of particle fluxes across the boundaries to the target and the bulk plasma. These coupled differential equations that describe the particle and power balance are solved for the ionization region defined by the distance from the target $z_{1}<z<z_{2}$ and the width of the race track $r_{\mathrm{c} 2}-r_{\mathrm{c} 1}$ where $r_{\mathrm{c} 1}<r_{\mathrm{c}}<r_{\mathrm{c} 2}$. In the two region model that was developed by Kozák and Pajdarováa ${ }^{256}$ the ionization region is assumed to be half a torus next to the target.

It is instructive to explore the terms that enter the energy and particle balance equations to understand the processes that dictate the HiPIMS discharge. The rate of change of the electron energy density has three contributions: the externally supplied electrical power input, the surface losses, and the electron energy loss due to inelastic collisions. This translates into the temporal development of the electron temperature which follows the rate equation,

$$
\begin{gathered}
\frac{3}{2} n_{e} \frac{d T_{e}}{d t}=F_{\mathrm{PWR}} \frac{I_{\mathrm{d}} V_{\mathrm{d}}}{e V_{\mathrm{IR}}}-\frac{T_{e}}{2}-\frac{\left(\Gamma_{\mathrm{Ar}+}+\Gamma_{\mathrm{M}+}\right) S_{\mathrm{IR}}}{V_{\mathrm{IR}}} \\
-\left(\mathcal{E}_{\mathrm{c}, \mathrm{Ar}}+\frac{3}{2} T_{\mathrm{e}}\right) k_{\mathrm{iz}} n_{\mathrm{e}}\left(n_{\mathrm{Ar}}+n_{\mathrm{Ar}, \mathrm{h}}\right) \\
-\left(\mathcal{E}_{\mathrm{c}, \mathrm{Ar}}+\frac{3}{2} T_{\mathrm{e}}\right) k_{\mathrm{iz}, \mathrm{m}} n_{\mathrm{e}} n_{\mathrm{Ar}}+\mathcal{E}_{\mathrm{dex}} k_{\mathrm{dex}} n_{\mathrm{e}} n_{\mathrm{Ar}} \\
-\left(\mathcal{E}_{\mathrm{c}, \mathrm{M}}+\frac{3}{2} T_{\mathrm{e}}\right) k_{\mathrm{iz}, \mathrm{M}} n_{\mathrm{e}} n_{\mathrm{M}}+\left(6-\frac{3}{2} T_{\mathrm{e}}\right) k_{\mathrm{p}} n_{\mathrm{M}} n_{\mathrm{Ar}}{ }^{\mathrm{m}},
\end{gathered}
$$

where $V$ denotes volume, $S$ surface, and

$$
\Gamma_{n n}=\frac{1}{S_{\mathrm{IR}}} \int n_{n n} u_{n n} d S_{\mathrm{IR}}
$$

is the averaged flux density of species $n n$ across the boundary between the ionization region and the bulk plasma, $k=\langle\sigma v\rangle$ is the rate coefficient, $\sigma$ is the cross section, $\Gamma$ is the flux, and $\mathcal{E}$ is the energy in $\mathrm{eV}$. The indices are IR (ionization region), D (discharge), M (metal), P (Penning ionization), $\mathrm{m}$ (metastable level), iz (ionization), dex (deexcitation), c (effective cost of ionization, the sum of elastic collisions energy losses per electron-ion pair created), Ar (cold argon ground state atoms), and Ar,h (hot argon atoms, returning from the target after recombination).

The particle balance equation for the $\mathrm{M}^{+}$ions is

$$
\begin{aligned}
\frac{d n_{\mathrm{M}}^{+}}{d t}= & k_{\mathrm{iz}, \mathrm{M}} n_{\mathrm{e}} n_{\mathrm{M}}+k_{\mathrm{P}} n_{\mathrm{M}} n_{\mathrm{Ar}} \\
& +k_{\text {chexc }} n_{\mathrm{M}} n_{\mathrm{Ar}+}-\frac{\Gamma_{\mathrm{M}}+S_{\mathrm{IR}}}{V_{\mathrm{IR}}} .
\end{aligned}
$$

The first three terms are the internal collision processes in the ionization region, electron impact ionization, Penning ionization, and charge exchange collisions with $\mathrm{Ar}^{+}$ions. The fourth term represents the surface losses of the metal ions. The particle balance equation for the neutral metal atoms is

$$
\begin{aligned}
\frac{d n_{\mathrm{M}}}{d t}= & -k_{\mathrm{iz}, \mathrm{M}} n_{\mathrm{e}} n_{\mathrm{M}}-k_{\mathrm{P}} n_{\mathrm{M}} n_{\mathrm{Ar}}-k_{\mathrm{chexc}} n_{\mathrm{M}} n_{\mathrm{Ar}}+ \\
& +\frac{\Gamma_{\mathrm{Ar}}+S_{\mathrm{RT}} Y_{\mathrm{tg}}+\Gamma_{\mathrm{M}}+S_{\mathrm{RT}} Y_{\mathrm{ss}}}{V_{\mathrm{IR}}}-\frac{\Gamma_{\mathrm{M} \text {,iff }}}{l}
\end{aligned}
$$

and $l=z_{2}-z_{1}$ is the thickness of the ionization region. The three collision terms are the same as in Eq. (46). The fourth term describes the flux of sputtered metal atoms into the ionization region which follows from the ion fluxes to the race track (with the surface area $S_{\mathrm{RT}}$ ), multiplied by the corresponding sputter yields. The last term describes the diffusion of metal atoms out of the ionization region.

The particle balance equation for the cold argon gas atoms is

$$
\begin{array}{r}
\frac{d n_{\mathrm{Ar}}}{d t}=-k_{\mathrm{iz}} n_{\mathrm{e}} n_{\mathrm{Ar}}-k_{\mathrm{exc}, \mathrm{m}} n_{\mathrm{e}} n_{\mathrm{Ar}}+k_{\mathrm{P}} n_{\mathrm{M}} n_{\mathrm{Ar}}{ }^{\mathrm{m}} \\
-k_{\mathrm{dex}} n_{\mathrm{e}} n_{\mathrm{Ar}^{\mathrm{m}}}+\frac{\Gamma_{\mathrm{Ar}, \text { diff }}}{L} \\
-\frac{\left(\left(\Gamma_{\mathrm{M}, 0}-\Gamma_{\mathrm{M}, \mathrm{diff}}\right)+\left(\Gamma_{\mathrm{M}^{+}, 0}-\Gamma_{\mathrm{M}^{+}, \text {diff }}\right)\left(S_{\mathrm{IR}}-S_{\mathrm{RT}}\right)\right.}{V_{\mathrm{IR}}} \\
\times \frac{m_{\mathrm{M}}}{m_{\mathrm{Ar}}} \times \frac{n_{\mathrm{Ar}}}{n_{\mathrm{Ar}}+n_{\mathrm{Ar}}} .
\end{array}
$$

The first four terms describe the collision losses due to electron impact ionization, electron impact excitation to metastable levels, and gains due to Penning ionization and electron impact deexcitation from the metastable level, and were discussed earlier. The second term of the second line describes the diffusional refill of argon from the surroundings, with a flux density $\Gamma_{\mathrm{Ar} \text {,diff }}$ that is determined by the gas temperature and the difference $\left(n_{\mathrm{Ar}, 0}-n_{\mathrm{Ar}}\right)$ in density. The last term describes the effect of the metal sputter wind on the argon gas, essentially reflecting momentum (or pressure) balance. Due to collisions with argon there is a reduction proportional to $\left(\Gamma_{\mathrm{M}, 0}-\Gamma_{\mathrm{M}, \text { diff }}\right)+\left(\Gamma_{\mathrm{M}^{+}, 0}-\Gamma_{\mathrm{M}^{+} \text {,diff }}\right)$ in the metal (both neutrals and ions) momentum flow from the ionization region to the bulk plasma, i.e., across the surface $\left(S_{\mathrm{IR}}-S_{\mathrm{RT}}\right)$. This is balanced by a corresponding outwards argon flux, taking into account the difference in mass, and also the fraction of the lost momentum that goes to metastable argon atoms.

These models provide a flexible tool to explore the discharge properties and the internal processes such as the temporal variations of the ionized fractions of the working gas and the sputtered vapor, the electron density and temperature, and the gas rarefaction and refill processes. ${ }^{31,255,256}$ The model calculations show that during the main pulse, $10-15 \mu$ s into the pulse until $100 \mu$ s into the pulse, the electron density increases and decays closely in phase with the discharge current. ${ }^{255}$ Furthermore, the calculations made for aluminum target show that the degree of argon ionization reaches $36 \%$, and it peaks later than the discharge current peak. This is due to extreme gas depletion, so that a given argon ion density corresponds to higher degree of ionization at the end of the pulse. The degree of metal ionization on the other hand has a broader and earlier peak, and a higher degree of ionization, $80 \%-85 \%$. The higher ionization fraction is a direct consequence of the lower ionization potential, $5.96 \mathrm{eV}$ for aluminum compared to $15.76 \mathrm{eV}$ for argon. It is also observed that electron impact ionization dominates the production of metal ions, with the contributions from Penning ionization and charge exchange to be almost negligible during the pulse-on time. Gas depletion reaches a value of over $96 \%$ and is significantly delayed in time with respect to 
the discharge current maximum. Model runs ${ }^{258}$ confirm the proposal by Lundin et al., ${ }^{193}$ that in HiPIMS the gas depletion due to ionization losses can dominate over the kick-out by the sputter wind (discussed in Sec. IV B), in contrast to what has been observed for dcMS. ${ }^{241}$ This model has also been used to explore the ionization fraction of various target metals in an HiPIMS discharge assuming typical values of $\sim 880 \mathrm{~V}$ (constant voltage) and current peak of $\sim 30$ A to generate approximately $100 \mu$ s discharge pulses at repetition frequency of $100 \mathrm{~Hz}$ on a $7.6 \mathrm{~cm}$ diameter target. ${ }^{31}$ The ionization fraction for Ti reaches $56 \%$ roughly $55 \mu$ s into the pulse, while $\mathrm{Cu}$ reaches an ionization fraction of $27 \%$. It can be seen that, although $\mathrm{Ti}$ has a higher ionization potential than $\mathrm{Al}$, the ionization fraction for $\mathrm{Ti}$ is higher $(56 \%$ at the peak) than for $\mathrm{Al}$ (49\% at the peak), since the electron impact ionization collision cross section is twice as large as that for $\mathrm{Al}$ and the mobility of $\mathrm{Ti}^{+}$being slightly lower. $\mathrm{Cu}$ on the other hand has higher ionization threshold than Ti or $\mathrm{Al}$ and a much lower electron impact ionization collision cross section, and exhibits lower ionization fraction.

\section{Transport of charged particles}

The motion of charged particles is one of the most challenging problems in HiPIMS plasma physics. The key parameters that determine the flow speeds $\mathbf{v}_{\mathrm{e}}$ and $\mathbf{v}_{\mathrm{i}}$ of electrons and ions in the plasma are the magnetic field $\mathbf{B}$; the gradient in electron pressure $\nabla p_{\mathrm{e}}$; the electric field which here is split up into a quasi-dc part denoted by $\mathbf{E}$ (which varies on the time scale of the HiPIMS pulse), and a high frequency $\mathbf{E}_{\mathrm{hf}}$ part associated with waves, turbulence, and anomalous resistivity and transport; the classical elastic mean free path $\lambda_{\text {coll }}$ for collisions with charged and neutral gas particles; and the characteristic length scale $l_{\mathrm{c}}$ of the device. In sputtering magnetrons the inequalities $r_{\mathrm{ce}} \ll l_{\mathrm{c}} \ll r_{\mathrm{ci}}$ generally hold as discussed in Sec. II A, meaning that the electrons are magnetized while the ions are practically unmagnetized. Ion-neutral mean free paths are found in ranges from $\lambda_{\text {coll }} \ll l_{\mathrm{c}}$ to $\lambda_{\text {coll }}>l_{\mathrm{c}}$ depending on apparatus size and gas pressure. However, for the electron flow and transport, classical collisions are usually less important than anomalous collisions, mediated by the high frequency $\mathbf{E}_{\mathrm{hf}}$ fields. In a fluid description these can be represented by an anomalous effective ion-electron momentum exchange time constant $\tau_{\text {eff }}$ which, however, only applies to the cross-B component of the electron-ion relative motion (alternatively expressed, the resistivity is a tensor $\bar{\eta}$ with a small field-aligned component). Often, $\tau_{\text {eff }}$ is called the effective collision time.

We attempt to introduce the mechanisms involved in the motion of charged particles in Table IV in such a way that they appear in a natural sequence. For each new mechanism we will refer to the corresponding box in Table IV. Three types of charged species will be treated: working gas ions, in the text denoted as $\mathrm{Ar}^{+}$, ions of the sputtered vapor, $\mathrm{M}^{+}$, and electrons, e. The motion of each type of species in the magnetic trap (the IR plus the BP regions, see Fig. 16) is discussed in three physical situations of increasing complexity, which also means increasing closeness to a real HiPIMS dis- charge: zero $\mathbf{E}$ field, finite $\mathbf{E}$ field, and anomalous resistivity. For the following discussion we define $z=0$ at the target surface and the $+z$ direction is away from the target.

\section{1. $\mathrm{Ar}^{+}$ions, zero E field (Table IV, box 1)}

A high return probability of the $\mathrm{Ar}^{+}$ions to the target $\beta_{\mathrm{g}}$ is beneficial for sustaining the discharge. The physical reason is that upon bombarding the target the ions lead to secondary emission of electrons which, after acceleration across the sheath, provide the energy needed for ionization in the plasma. From the discharge energy budget point of view, this energy source is proportional to the return probability $\beta_{\mathrm{g}}$. Due to the large cross section for resonant charge exchange collisions, in the first approximation the ion motion for zero $\mathbf{E}$ field can be treated as diffusion through the background gas, with a flux given by Ficks law, ${ }^{49}$

$$
\Gamma_{\text {diff }}=-D_{\text {diff }} \nabla n_{\mathrm{Ar}^{+}} .
$$

However, both the neutral gas and the $\mathrm{Ar}^{+}$ions are, in addition, moving away under the action of the sputter wind (including fast, recombined, argon reflected from the target surface). This gas flow speed must be added to the diffusion flow speed. In the case of significant gas rarefaction, the problem becomes more complicated because the collision dominated assumption might no longer be valid.

\section{2. $\mathrm{Ar}^{+}$ions, finite $\mathrm{E}$ field (Table IV, box 4)}

As discussed in Sec. III A there is usually an electric field extending into the plasma and directed toward the target, here denoted $E_{\mathrm{z}}$. In such a situation there are two simple extreme cases. In the collision-free extreme the $\mathrm{Ar}^{+}$ions move in ballistic orbits and will end up on the target if, at their point of ionization, their kinetic energy in the $+z$ direction (away from the target) is lower than the remaining potential hill $q_{\mathrm{i}} \Delta \Phi$ they have to climb in the $+z$ direction. In the collision dominated case the electric mobility drift $u_{z}=\mu_{\mathrm{i}} E_{\mathrm{z}}$ is added to (and directed against) the outwards flow speeds from the diffusion and the rarefaction flows treated earlier. They will move toward the target if they become ionized in a region where this total drift has a negative sign. Although exact calculations of the $\mathrm{Ar}^{+}$-ion drift speeds remain to be done we propose, based on estimates of the energies and drift speeds involved in the diffusion and rarefaction flow mechanisms earlier, that an electric potential $\Phi_{\mathrm{IR}}$ of a few volts across the ionization region should be sufficient for a rather high $\mathrm{Ar}^{+}$return probability, say, $0.7<\beta_{\mathrm{g}}$.

\section{3. $M^{+}$-ions, zero E field (Table IV, box 2)}

If there is no electric field in the plasma, the $\mathrm{M}^{+}$ions behave as neutrals of the same species (disregarding the difference in the elastic cross sections). The motion of the neutral sputtered species has been studied by Msieh et al. ${ }^{259}$ by Monte Carlo simulations. Test particles were injected at $z=0$ with a sputtered energy and angular distribution, and followed in time in a uniform neutral gas. The resulting velocity distribution was extracted as function of $z$. Two 
distinct populations were identified: one not collided population retaining the original sputter distribution, with density decreasing approximately as $\exp \left(-z / \lambda_{\text {coll }}\right)$, and another collided population, approximately thermal with the ambient gas temperature. In the zero $\mathbf{E}$ field case, the not collided $\mathrm{M}^{+}$-ion population does not return to the target, while the collided population will behave as the $\mathrm{Ar}^{+}$ions in zero $\mathbf{E}$ field treated earlier, moving according to the combined mechanisms of diffusion and rarefaction flow.

\section{4. $\mathrm{M}^{+}$ions, finite $\mathrm{E}$ field (Table IV, box 5)}

This is the key combination to understand for the return probability $\beta_{\mathrm{t}}$ of the $\mathrm{M}^{+}$ions, the major unknown in both the material pathway and the IRM models, and also probably one of the major reasons for the reduced deposition rate discussed in Sec. III C. Each ion has, at the time and place of ionization, the same initial velocity $\mathbf{u}_{\mathrm{i} 0}$ as the neutral had (we distinguish here between individual particle velocities $\mathbf{u}$ and flow velocities $\mathbf{v})$. After ionization, and until the next collision, the ions follow ballistic orbits where $d \mathbf{u}_{\mathrm{i} 0} / d t$ (i.e., not $\mathbf{u}_{\mathrm{i}}$ ) is determined by the local electric fields $\mathbf{E}$. A fluid description cannot accurately describe such motion. However, a few observations can be made. The collided, thermal, part of the $\mathrm{M}^{+}$-ion distribution will behave much as the $\mathrm{Ar}^{+}$population discussed earlier and is therefore expected to return to the target already at potentials of a few volts. Uncollided $\mathrm{M}^{+}$ions; on the other hand, start with $\mathbf{u}_{\mathrm{i} 0}$ directed away from the target, and with the sputtered energy distribution [Eq. (14)]. Their point of ionization is furthermore determined by their velocity: those with low initial velocity $u_{z}$ spend more time close to the target, and are therefore on the average ionized closer to it. These slow ions both have a lower directed kinetic energy in the $+z$ direction, and a larger average potential hill $q_{\mathrm{i}} \Delta \Phi$ to climb, which creates a high-energy-pass filtering effect. The average point of ionization also depends on the time during the pulse: at peak current, the plasma density is higher, and ionization occurs closer to the target as the mean free path for electron impact ionization changes from a very small value to several centimeters.

In summary, the ion transport speeds can generally be expected to be very complicated functions both of the local parameters and the time histories of the individual particles.

Even if the electric potential profile $\Phi(\mathbf{r}, t)$ were known, calculations of the ion transport velocity would require PIC simulations or at least Monte Carlo simulations. However, the above-presented discussion demonstrates the possibility that there might exist a high deposition rate window somewhere in the range $1<\Phi_{\mathrm{IR}}<20 \mathrm{~V}$, in which the potential is high enough to give the high $\beta_{\mathrm{g}}$ needed to sustain the discharge, but so low that it lets most of the not collided part of the $\mathrm{M}^{+}$population escape to the bulk plasma.

\section{Electron transport along B (Table IV, box 3)}

The magnetic field aligned component $v_{\mathrm{e} \|}$ of the electron drift speed $\mathbf{v}_{\mathrm{e}}$ is not determined by the local plasma parameters. The reason is that electrons, with their light mass, easily respond by a redistribution along $\mathbf{B}$ to departure from the equilibrium state in which the pressure gradient is balanced by the electric volume force,

$$
\nabla p_{\mathrm{e} \|}=e n_{\mathrm{e}} E_{\|}
$$

If there is a loss (or gain) of charge in one part of a flux tube, electrons are redistributed along it and modify the electric field (through Poissons equation) so that Eq. (50) is maintained. $v_{\mathrm{e} \|}$ is therefore driven by the global cross- $\mathbf{B}$ current pattern. The collisional parallel resistivity can generally be neglected in this process. In a homogeneous plasma, $\nabla p_{\mathrm{e} \|}=0$, giving $E_{\|}=0$, and the magnetic field lines are equipotentials. However, in the dynamic and inhomogeneous HiPIMS pulses, the full expression (50) will be needed, particularly in the region penetrated by high energy secondary electrons accelerated across the sheath.

\section{Electron transport across B-classical mobility (Table IV, boxes 3 and 6)}

The perpendicular part of $\mathbf{v}_{\mathrm{e}}$ is split up into two parts depending on the direction with respect to the net cross-B force,

$$
\mathbf{F}_{\mathrm{e} \perp}=\left(-\nabla p_{\mathrm{e}}-e n_{\mathrm{e}} E\right)_{\perp} .
$$

\begin{tabular}{|c|c|c|c|}
\hline Physical situation & Working gas ions, $\mathrm{Ar}^{+}$ & Ions of the sputtered vapor, $\mathbf{M}^{+}$ & Electrons \\
\hline \multirow[t]{4}{*}{ Zero E-field } & $(1)$ & $(2)$ & (3) \\
\hline & Diffusion & Sputter flow & Force balance $\| \mathbf{B}$ \\
\hline & Rarefaction flow & Diffusion & Diamagnetic drift $\perp \mathbf{B}$ \\
\hline & & Rarefaction flow & Cross-B classical diffusion $\perp \mathbf{B}$ \\
\hline \multirow[t]{4}{*}{ Finite E-field } & (4) & $(5)$ & $(6)$ \\
\hline & All above plus & All above plus & All above plus \\
\hline & Mobility drift & Ballistic energy filter & Hall drift $\perp \mathbf{B}$ \\
\hline & & Mobility drift & Classical Pedersen conductivity $\perp \mathbf{B}$ \\
\hline \multirow[t]{3}{*}{ Anomalous resistivity } & $(7)$ & $(8)$ & (9) \\
\hline & All above plus & All above plus & All above plus \\
\hline & Azimuthal drag & Azimuthal drag & Cross-B anomalous mobility $\perp \mathbf{B}$ \\
\hline
\end{tabular}

TABLE IV. Overview of the mechanisms that influence the motion of charged particles in the magnetic trap (the ionization region plus the bulk plasma). 
Let us consider a cylindrical magnetron with azimuthal symmetry, where $\mathbf{F}_{\mathrm{e} \perp}$ lies in the $(r, z)$ plane. In the $\mathbf{F}_{\mathrm{e} \perp} \times B$ direction, there is an azimuthal drift speed,

$$
v_{\mathrm{e} \phi}=\frac{F_{\mathrm{e} \perp}}{e n_{\mathrm{e}} B} \frac{\omega_{e}^{2} \tau_{c}^{2}}{1+\omega_{\mathrm{e}}^{2} \tau_{\mathrm{c}}^{2}},
$$

where $\tau_{\mathrm{c}}$ is the classical collision time due to electron-ion (Coulomb) and electroneutral collisions and $\omega_{\mathrm{e}}$ is the electron cyclotron angular frequency [given by Eq. (7)]. The dimensionless cross-B transport parameter $\omega_{\mathrm{e}} \tau_{\mathrm{c}}$ is common for electric conductivity, electron diffusion, diamagnetic drift, electron mobility, and the magnetic field diffusion into a plasma. ${ }^{260}$ It is often referred to as the Hall parameter. In the collision-free limit $\left(\omega_{\mathrm{e}} \tau_{\mathrm{c}} \rightarrow \infty\right)$ the sum of the Hall drift, Eq. (9), and the diamagnetic drift, Eq. (10), are recovered from Eqs. (51) and (52).

Since the ions are unmagnetized, they have no corresponding drift motion and this drift gives an azimuthal current density $\mathbf{J}_{\mathrm{e}, \phi}=-e n_{\mathrm{e}} v_{\mathrm{e}}{ }^{\phi}$. In the direction along $\mathbf{F}_{\mathrm{e} \perp}$ the electron drift component has the value

$$
v_{\mathrm{e}}(r, z) \frac{F_{\mathrm{e} \perp}}{e n_{\mathrm{e}} B} \frac{\omega_{\mathrm{e}} \tau_{\mathrm{c}}}{1+\omega_{\mathrm{e}}^{2} \tau_{\mathrm{c}}^{2}} .
$$

An important relation, first proposed by Rossnagel and Kaufman, ${ }^{68}$ can be derived for the case when the pressure term in $\mathbf{F}_{\mathrm{e} \perp}$ is negligible. In that case the currents (i.e., the electron drifts in the ion rest frame) across $\mathbf{B}$ are given by the classical Hall and Pedersen conductivities, which can be obtained from the generalized Ohms law as functions of the Hall parameter $\omega_{\mathrm{e}} \tau_{\mathrm{c}}$. In the plasma bulk the electrons have to move across the magnetic field lines in order to arrive at the anode. The electric-field-driven part of the cross-B discharge current density and the electric field are related through the generalized Ohm's law,

$$
E_{\perp}=\frac{J_{\mathrm{d} \perp}}{\sigma_{\mathrm{P}}}=J_{\mathrm{d} \perp}\left[\frac{e n_{\mathrm{e}}}{B} \frac{\omega_{\mathrm{e}} \tau_{\mathrm{c}}}{1+\left(\omega_{\mathrm{e}} \tau_{\mathrm{c}}\right)^{2}}\right]^{-1},
$$

where $\sigma_{\mathrm{P}}$ is the Pedersen conductivity. In the bulk plasma the current is carried mainly by electrons and we can write $^{168}$

$$
\frac{J_{\mathrm{e}, \phi}}{J_{\mathrm{d} \perp}}=\frac{E_{\perp} \sigma_{\mathrm{H}}}{E_{\perp} \sigma_{\mathrm{P}}}=\frac{E_{\perp} \frac{e n_{\mathrm{e}} \omega_{\mathrm{e}}^{2} \tau_{\mathrm{c}}^{2}}{B 1+\left(\omega_{\mathrm{e}} \tau_{\mathrm{c}}\right)^{2}}}{E_{\perp} \frac{e n_{\mathrm{e}} \omega_{\mathrm{e}} \tau_{\mathrm{c}}}{B 1+\left(\omega_{\mathrm{e}} \tau_{\mathrm{c}}\right)^{2}}}=\omega_{\mathrm{e}} \tau_{\mathrm{c}}
$$

where $J_{\mathrm{e}, \phi}$ is the azimuthal current density, $J_{\mathrm{d} \perp}$ is the discharge current density, and $\sigma_{\mathrm{H}}$ is the Hall conductivity. Thus, the Hall parameter can be obtained from a measurement of the current ratio $J_{\mathrm{e}, \phi} / J_{\mathrm{d} \perp}$.

\section{Electron transport across $B$-anomalous resistivity (Table IV, box 9)}

Classical theory of diffusion and electrical conductivity where collisions move electrons across the magnetic field lines results in values that scale as $1 / B^{2}$. A more rapid or anomalous loss of plasma across magnetic field lines is caused by microinstabilities and is referred to as Bohm diffusion. Then the diffusion scales as $1 / B$ or as described by the semiempirical Bohm diffusion coefficient, ${ }^{73,261}$

$$
D_{\mathrm{B}}=\frac{1}{16} \frac{k T_{\mathrm{e}}}{e B} .
$$

Bohm diffusion can be formally ascribed to anomalous collisions, with an effective collision time $\tau_{\text {eff }}$. The empirically found constant 16 in Eq. (56) is, in this description, the anomalous Hall parameter, i.e., $\left(\omega_{\mathrm{e}} \tau_{\mathrm{eff}}\right)_{\mathrm{Bohm}}=16$. This approach has been generalized to include conductivity, mobility, and magnetic field diffusion, ${ }^{68,260,262}$ wherein also the relation (55) has been shown to apply for pressure driven (i e, diamagnetic) currents. Thus, a determination of $J_{\mathrm{e}, \phi} / J_{\mathrm{d} \perp}$ gives a direct measure of $\omega_{\mathrm{e}} \tau_{\mathrm{eff}}$, and thereby all transport parameters needed for fluid modeling: $\mu_{\perp}, \eta_{\perp}, \sigma_{\mathrm{H}}, \sigma_{\mathrm{P}}, D_{\perp}$, and the magnetic field diffusion constant. For definitions and relations to $\omega_{\mathrm{e}} \tau_{\mathrm{eff}}$ of these, see Brenning et al. ${ }^{260}$ The azimuthal drift currents in a circular planar dcMS have been measured for a range of parameters and values in the range $J_{\mathrm{e}, \phi} / J_{\mathrm{d} \perp} \sim 3-35$ have been reported. ${ }^{68,74,263,264}$ The drift current was found to vary roughly linearly with the discharge current. Furthermore, none or only a weak dependence on the gas species or the cathode target material is observed. ${ }^{68}$ It turns out that electron cross-B transport in the HiPIMS discharge is much faster than classically predicted through collisions and also faster than Bohm diffusion. Brenning et al. ${ }^{260}$ show that the diffusion coefficient is roughly a factor of 5 greater than what the Bohm diffusion would predict. This faster than Bohm diffusion range is $1.5<J_{\mathrm{e}, \phi} / J_{\mathrm{d} \perp}<5.5$. The early measurements by Bohlmark et $a .^{264}$ indicated that the ratio between the azimuthal current density $J_{\mathrm{e}, \phi}$ and the discharge current $J_{\mathrm{d} \perp}$ in an HiPIMS discharge is $J_{\mathrm{e}, \phi} / J_{\mathrm{d} \perp} \sim 2$. More recently the spatial and temporal variation of the internal current densities $J_{\mathrm{e}, \phi}$ and $J_{\mathrm{d} \perp}(z)$ have been measured by a Rogowski coil. ${ }^{265}$ These measurements indicate a variation of the transport parameter $J_{\mathrm{e}, \phi} / J_{\mathrm{d} \perp}$ over time and space. The low values of $J_{\mathrm{e}, \phi} / J_{\mathrm{d} \perp} \approx 2$ are observed $7-8 \mathrm{~cm}$ from the target surface. Closer to the target $J_{\mathrm{e}, \phi} / J_{\mathrm{d} \perp}$ increases with decreasing distance approaching the values expected for Bohm diffusion. There are small variations in $J_{\mathrm{e}, \phi} / J_{\mathrm{d} \perp}$ with time, values stay within a factor of 2 from the average value. These results are shown in Fig. 17, which shows $J_{\mathrm{e}, \phi} / J_{\mathrm{d} \perp}$ above the race track for times $30,60,85,100$, and $130 \mu$ s into the pulse. The low value of $J_{\mathrm{e}, \phi} / J_{\mathrm{d} \perp}$ observed for an HiPIMS discharge indicates a much more efficient electron transport across the magnetic field lines than for a dcMS, which is a result of decreased transverse resistivity $\eta_{\perp}$ and thereby increased cross-B conductivity and diffusion of electrons.

\section{8. $\mathrm{Ar}^{+}$and $\mathrm{M}^{+}$ions-anomalous resistivity (Table IV, boxes 7 and 8)}

The high energy tails observed in the IEDF in the HiPMS discharge, with measured energies up to $100 \mathrm{eV}$ (see Figs. 9 and 10) is a desirable feature for film growth, but the 


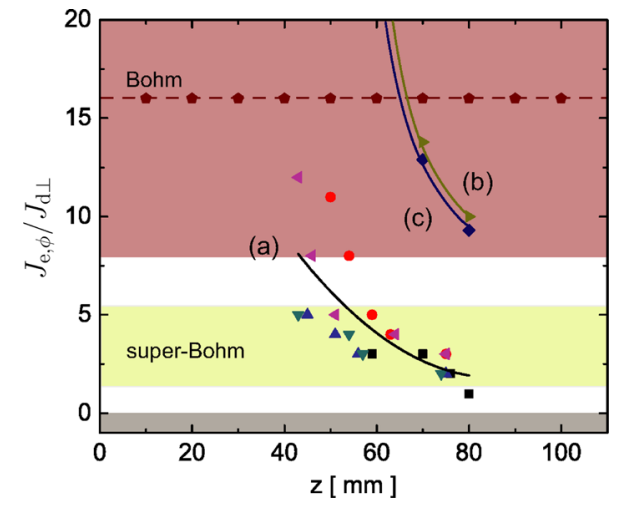

FIG. 17. (Color online) Measurements of $J_{\mathrm{e}, \phi} / J_{\mathrm{d} \perp} \approx \omega_{\mathrm{e}} \tau_{\mathrm{eff}}$ above the race track, at $30,60,85,100$, and $130 \mu$ s into the pulse vs distance from the cathode surface. The curve marked (a) shows the average of the experimental data. For reference, two values of classical $J_{\mathrm{e}, \phi} / J_{\mathrm{d} \perp}$ are also given: classical electron-neutral collisions (b), and combined electron-neutral and Coulomb collisions (c), as well as the Bohm-value $J_{\mathrm{e}, \phi} / J_{\mathrm{d} \perp}=16$ (dashed). The Bohm region, $8<J_{\mathrm{e}, \phi} / J_{\mathrm{d} \perp}<30$, as well as the faster-than-Bohm region (superBohm), $1.5<J_{\mathrm{e}, \phi} / J_{\mathrm{d} \perp}<5.5$, appear as shaded areas. From Lundin et al. (Ref. 265).

acceleration of these ions is at present not understood and any model for ion transport that does not include this feature must be regarded as incomplete. Lundin et al. ${ }^{167}$ argued that this ion energization is associated with the anomalous resistivity effect that gives the efficient cross-B electron transport. This claim was in Lundin et al. ${ }^{167}$ supported by experimental data where a mass spectrometer showed a much more pronounced high energy tail in the IEDF in the expected azimuthal direction, as indicated in Fig. 18 and discussed in Sec. III B. The proposed mechanism is also shown in Fig. 18 and is based on results from PIC simulations of the wave structure in the modified two stream instability (MTSI). ${ }^{266}$ The instability, when driven by an azimuthal current $J_{\mathrm{e}, \phi}$, sets up a wave structure with the wave vector $k_{\phi}$, and in which the wave electric field $E_{\mathrm{W}}$ and the density perturbations ( $\delta n_{\mathrm{e}}=\delta n_{\mathrm{i}}$ due to quasi-neutrality) are correlated in such a fashion that there is a net azimuthal force $F_{\text {ei }}= \pm\left\langle e n_{\mathrm{e}} E_{\mathrm{W}}\right\rangle$ between ions an electrons. The force is directed along $J_{\mathrm{e}, \phi}$ and gives the anomalous resistivity effect. However, besides the action on the electrons that facilitates the radial transport, there must be an equal and opposite reaction on the ions, i.e., a net average drag against the direc-

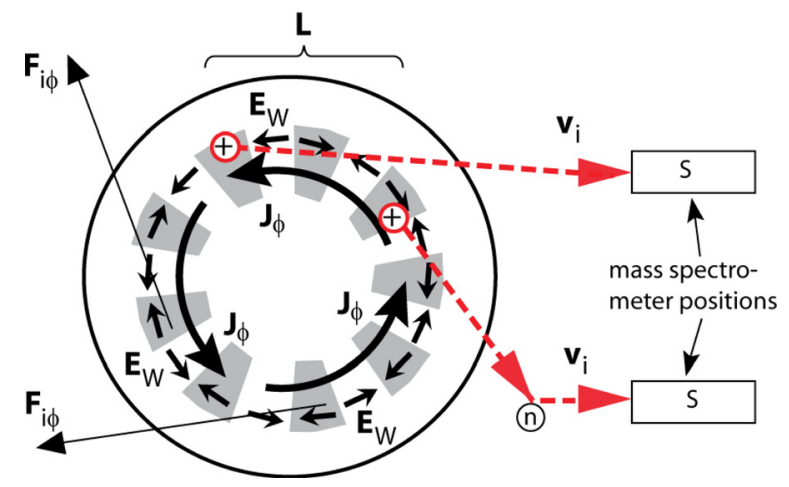

FIG. 18. (Color online) Azimuthal ion acceleration, and the mechanism proposed by Lundin et al. ${ }^{168}$ tion of $J_{\mathrm{e}, \phi}$. The two-stream instability is driven by the relative drifts between electrons $v_{\mathrm{e}}$ and ions $v_{\mathrm{i}}$ in the plasma, i.e., $v_{\text {rel }}=v_{\mathrm{i}}-v_{\mathrm{e}}$, in the presence of a magnetic field component perpendicular to this relative drift. This is the case for the circulating azimuthal current in the magnetic field trap of a magnetron above the target in an HiPIMS discharge. ${ }^{264}$ Because the ion gyro radii in magnetron sputtering discharges are typically larger than the spatial dimension of the plasma, only the electrons are magnetized and take part in this azimuthal drift. Lundin et al. ${ }^{262}$ estimate the individual contributions of the various drift terms to the total azimuthal current. They find that the Hall drift [Eq. (9)] and the diamagnetic drift [Eq. (10)] are in the same direction and combine to an azimuthal drift speed exceeding the MTSI threshold. This approach was further developed by Poolcharuansin et al., ${ }^{169}$ who added a drag force term, accounting for the azimuthal anomalous-resistive drag force, to the ion equation of motion which was solved numerically. It was shown that a fraction of the circulating ion flux, which does not suffer from collisions, can then overcome a radial electric field and leave the discharge volume in the tangential direction. Results obtained for elevated pressures indicate that the sideway transport of ions is increasingly influenced by scattering of ions out of the discharge volume.

Previous investigations of the MTSI have shown that the result will be large oscillations in the electric field, which are often correlated with the plasma density, resulting in a net transport of electrons perpendicular to both $J_{\mathrm{e}, \phi}$ and $\mathbf{B}$ while the ions are too heavy to follow this motion. ${ }^{266}$ Measurements of oscillating electric fields in the megahertz range in this and other devices ${ }^{262,267}$ are also consistent with the MTSI as discussed earlier. Lundin et al. ${ }^{262}$ demonstrated that the frequency dependence on the ion mass and the magnetic field strength corresponds to lower hybrid oscillations when the fraction of the ions of the sputtered vapor is roughly $80 \%$ of the total ion density. Besides the MTSI, also other frequency ranges and instabilities have been discussed. Oscillations have been observed in dcMS (Refs. 268 and 269) as well as in HiPIMS plasmas, ${ }^{16,179}$ and sometimes put in the context of fast electron transport. ${ }^{269,270}$

Another type of process that can facilitate radial electron cross-B transport is spoke formation. Spoke formation has been reported during the ignition and growth phases of pulsed homopolar cross-B discharges. ${ }^{271-273}$ Such discharges have a different discharge geometry than sputtering magnetrons, but are topologically similar. A spoke is a rotating radial channel of enhanced plasma density, within which an azimuthal electric field gives a radial Hall drift of the electrons that carries the discharge current. There are indications that spoke formation should be considered also in HiPIMS. Optical studies of a preionized HiPIMS discharge show that azimuthal inhomogeneity or plasma bunches are formed and rotate clockwise with a linear velocity $1 \mathrm{~cm} / \mu \mathrm{s}$, in the direction of the electron $\mathbf{E} \times \mathbf{B}$ drift. ${ }^{59}$ The plasma is more homogeneous when the magnetron discharge is operated in an unbalanced mode and the number of plasma bunches increases with increased current amplitude. This phenomena has been explored further by Anders et al. ${ }^{274}$ 
which argues that this is caused by an ionization instability and that each of these dense plasma zones is associated with an azimuthal electric field $\mathbf{E}_{\theta}$ which breaks the magnetic confinement of the electrons due to an $\mathbf{E}_{\theta} \times \mathbf{B}$-drift component, which is in the axial direction, away from the target.

\section{E. Modes of operation}

Here we explore the physical mechanisms at work during different stages of the discharge pulse, such as process gas dynamics, sputtering, ionization, and current distribution. Of particular interest is how internal pulse features such as the gas rarefaction, the degree of self-sputtering, and the degree of ionization of the sputtered species, can be influenced by the choice of the pulse length and the applied power density. For simplicity we here assume square shaped voltage pulses. Figure 19 shows typical pulse shapes of the discharge current, when the applied power density is varied from the middle of the MPP range of Fig. 1, up to the higher end of the HiPIMS range. In Fig. 19 the HiPIMS discharge pulses have been divided into five different phases during an approximately $300 \mu$ s long pulse. We have for simplicity only chosen to display the discharge current, which in this example is shown to develop along different pathways generally characterized by an initial peak followed by a more or less stable current plateau (bottom current curves in Fig. 19), or an initial peak followed by a second increase of the discharge current (top current curves in Fig. 19). Note that the discharge current amplitude, the point in time for reaching the peak current, current transitions, etc., for each of these different pathways is likely to change somewhat depending on discharge conditions, such as target material, gas composition, repetition frequency, the magnetic field strength, and other factors, ${ }^{17,227,275}$ and should therefore not be taken as exact values. We propose that such curves are suitably categorized according to the degree of self-sputtering during the plateau/ runaway phase (i.e., during phase 4 in Fig. 19), where we

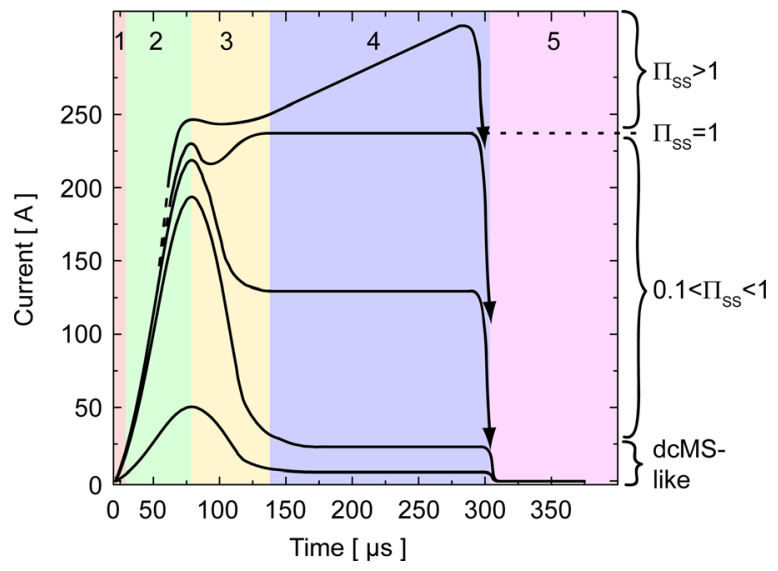

FIG. 19. (Color online) Schematic illustration of the HiPIMS discharge current divided into five different phases. The bottom two curves display an approximately $300 \mu$ s long current pulse, where the current decays after an initial peak at around $80 \mu$ s mainly due to depletion of the working gas, followed by a current plateau. The top two curves illustrate the onset of selfsputtering, where the current may reach a second maximum before the pulse is switched off. The middle curve displays an intermediate state due to partial self-sputtering. use for quantification the self-sputtering parameter $\Pi_{\mathrm{ss}}$ of Eq. (16). Huo et al. ${ }^{258}$ used the IRM to model experiments with long pulses ${ }^{212}$ that covered power densities in the range $1-10 \mathrm{~kW} / \mathrm{cm}^{2}$. The model showed that $\Pi_{\mathrm{ss}}=0$ for the lowest power densities, obtained when the applied pulse was slightly above the breakdown voltage, and that for higher power densities was an almost linear increase of $\Pi_{\mathrm{ss}}$ with $I_{\text {d,plateau. }}$ The bottom curve in Fig. 19 represents a range of low self-sputtering, say, $\Pi_{\mathrm{ss}}<0.1$. In this range we propose the discharge physics in the plateau/runaway phase to be dcMS-like. Lundin et al. ${ }^{193}$ describe a case where fairly stable plateau currents (phase 4) values agree with the currents in dcMS at the same discharge potential. They point out that short $(<100 \mu \mathrm{s})$ HiPIMS pulses give higher current for a given voltage because they only exhibit the initial transient. The middle range of power densities, with $0.1<\Pi_{\mathrm{ss}}<1$, represents discharges that we categorize as being partially self-sputtering. For increasing power in this range, there is experimental evidence that the degree of ionization of the sputtered species increases, and also the fraction of doubly ionized sputtered species. ${ }^{212}$ The top curve in Fig. 19 represents runaway self-sputtering which requires $\Pi_{\mathrm{ss}}>1$ and therefore, from Eq. (16), a self-sputter yield $Y_{\mathrm{ss}}>1 /\left(\alpha \beta_{\mathrm{t}}\right)>1$ or through recycling of ions of the working gas to fulfill Eq. (17). Runaway self-sputtering has been demonstrated for a number of target materials, including $\mathrm{Cu}, \mathrm{Nb}$, and $\mathrm{Ti}$, but also $\mathrm{C}$ and $\mathrm{Si}^{136,212,275}$ and for reactive sputtering as well. ${ }^{227}$ The current runaway (when $\Pi_{\mathrm{ss}}>1$ ) is sometimes more abrupt than demonstrated by the uppermost curve in Fig. 19 (see, e.g., Refs. 212 and 227). In the following an overview is given of the five phases shown in Fig. 19 based on experimental data from the partial self-sputtering range.

Phase 1 (ignition) covers approximately the first $10 \mu$ s of the discharge pulse, which constitutes the ignition phase during which there is negligible plasma in the bulk volume of the discharge chamber. The discharge will likely ignite as a localized glow discharge at the target substrate in the vicinity of the anode ring, where the vacuum electric field is the strongest, which thus leads to a rather small current in the radial direction in the case of a circular magnetron. ${ }^{265}$ Time resolved tunable diode-laser absorption spectroscopy measurements by Vitelaru et $a .^{247}$ show that there is a very strong increase in the emission from the metastable working gas atoms $\left(\mathrm{Ar}^{\mathrm{m}}\right)$ during phase 1, which is seen in Fig. 15. As previously described in Sec. III A, Poolcharuansin and Bradley ${ }^{146}$ detected a short burst of hot electrons $(70-100 \mathrm{eV})$ only within the first $10 \mu \mathrm{s}$ of the HiPIMS pulse promoting electron impact excitation, which would explain the great increase in the number of $\mathrm{Ar}^{\mathrm{m}}$ present. Also the neutral gas temperature starts to increase reaching around $600-800 \mathrm{~K}$ (Fig. 15), ${ }^{247}$ which is likely due the increasing amount of collisions taking place. Using optical emission spectrometry Hála et al. ${ }^{136}$ also find that there is an initial strong increase in the emission from the neutral working gas as seen in Fig. 20

Phase 2 (current rise) displays the commonly seen strong initial current increase after the bulk plasma breakdown. Secondary electrons as well as electrons created in the 


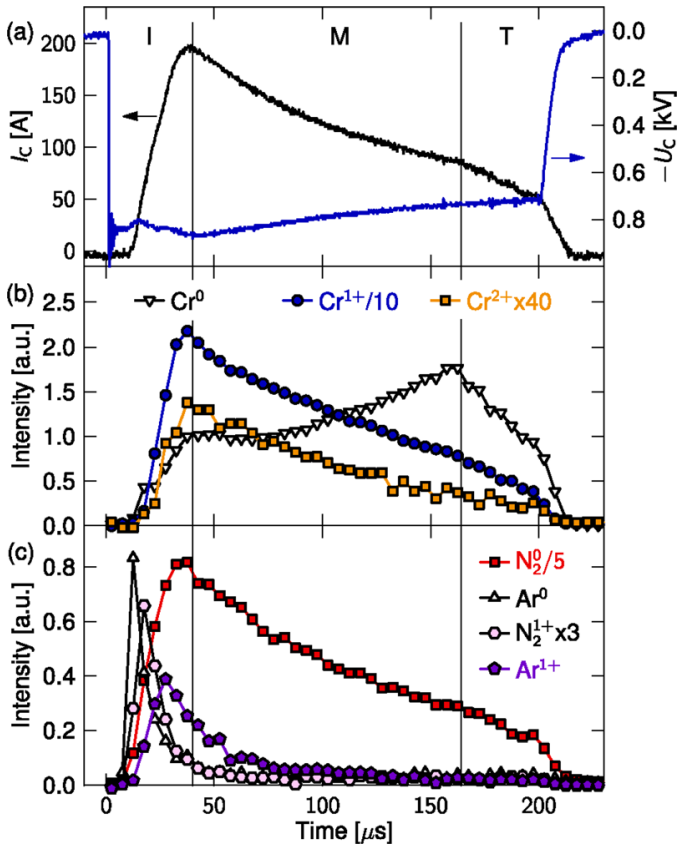

FIG. 20. (Color online) (a) Waveforms of the current and voltage, (b) the optical emission from $\mathrm{Cr}, \mathrm{Cr}^{+}$, and $\mathrm{Cr}^{2+}$, and (c) the optical emission from $\mathrm{N}_{2}$, $\mathrm{Ar}, \mathrm{N}_{2}^{+}$, and $\mathrm{Ar}^{+}$for an HiPIMS discharge operated on $\mathrm{N}_{2} / \mathrm{Ar}$ (1:1) mixture at $1.3 \mathrm{~Pa}$ with $\mathrm{Cr}$ target. The three phases observed are the ignition phase (I), the high-current metal-dominated phase (M), the transient phase (T), and the low-current gas dominated phase (G). From Hála et al. (Ref. 136).

ionization region close to the target are accelerated out along the magnetic field lines into the bulk volume and begin to ionize the neutral gas resulting in strong axial current (perpendicular to the target surface) toward the edge and the center of the target, where the magnetic field lines intersect the cathode. ${ }^{265} \mathrm{~A}$ dense plasma torus above the target race track is now also developing. ${ }^{265}$ At this stage the metal atom and argon metastable densities build up, but at the same time $\mathrm{Ar}^{\mathrm{m}}$ loss processes, such as Penning ionization of the sputtered vapor atoms as well as electron impact ionization of neutral gas atoms, will come into play. ${ }^{247}$ Furthermore, electron impact excitation and ionization of sputtered material atoms will cool the effective electron temperature, since the ionization potential of most sputtered materials is well below that of Ar. Vitelaru et al. ${ }^{247}$ report on a peak in the $\mathrm{Ar}^{\mathrm{m}}$ density during this phase as seen in Fig. 15, which is due to a balance between the ongoing production and loss mechanisms. Costin et al. ${ }^{276}$ use a fast time resolved 2D imaging to explore short pulses. They follow the emission of $\mathrm{Ar}$ and $\mathrm{Al}$ lines looking at the $\mathrm{Al}$ target during a $4 \mu$ s pulse. After a $0.5 \mu$ s delay they see a fast rise in the Ar emission lines to a peak at $1.2 \mu$ s into the pulse which coincides with a rapid expansion of the torus. The $\mathrm{Al}$ lines exhibit the same temporal behavior as the discharge current, a slow increase in intensity to a maximum $2.8 \mu$ s into the pulse and the $2 \mathrm{D}$ images show a narrow torus limited to the race track, at roughly constant width throughout the entire pulse.

Phase 3 (gas depletion). As the peak of the HiPIMS discharge current is approached there is a strong decrease in the emission from metastable argon atoms while the neutral ar- gon temperature $T_{\mathrm{Ar}}$ continues to increase, see Fig. 15 (note that the authors assume $T_{\mathrm{Ar}} \sim T_{\mathrm{Ar}}^{\mathrm{m}}$ since $\mathrm{Ar}^{\mathrm{m}}$ are produced from $\mathrm{Ar}$ and no great additional heating of the $\mathrm{Ar}^{\mathrm{m}}$ is expected). ${ }^{247}$ Also reported is a strong increase in the emission from neutrals and ions of the sputtered material, which is found to dominate the discharge between phases 2 and $3{ }^{136}$ At this stage the bulk plasma density has built up sufficiently to admit current closure cross the magnetic field lines (i.e., cross-B electron drift toward the anode/ground): first at larger distances from the target surface, but eventually the plasma density above the target race track is high enough so that this route is the easiest for the electron current to cross the magnetic field lines (cross-B drift), which results in a more evenly distributed axial current. ${ }^{265}$ The plasma potential has been found to be less negative compared to the ignition phase but might still reach down to $-40 \mathrm{~V}$ close to the target surface. ${ }^{200}$ During these conditions a strong reduction of working gas atom density occurs mainly due to (a) electron impact ionization of the working gas as a result of the much higher plasma densities during the peak of the HiPIMS discharge current as compared to for example dcMS (Ref. 255 ) and (b) gas rarefaction, where the heating is due to momentum transfer in collisions between the background gas and the increasing amount of sputter-ejected target atoms as well as reflected sputtering gas atoms, ${ }^{249}$ which also explains why $n_{\mathrm{Ar}}^{\mathrm{m}}$ decreases. Furthermore, the neutral gas temperature increase is likely due to collisions of argon atoms with the steadily increasing amount of sputtered material neutrals. ${ }^{247}$ Phase 3 does not exhibit a steady state as can be seen in Fig. 19, since the discharge either goes into the decay phase characterized by a decrease in current or a selfsputtering regime characterized by yet another current increase (or at least a sustained high current mode). As previously discussed in Sec. IV D, Anders et al. ${ }^{117}$ found that this phase could be dominated by self-sputtering.

Note that during phase 4 (plateau/runaway) there is also the possibility of having partial self-sputtering as described in the beginning of this section. This will increase the plateau current (middle current curve) compared to the dcMSlike case (bottom curve), since more charge carriers can be generated in the form of metal ions followed by the release of additional secondary electrons. However, this will only occur when having a significant fraction of ions with high enough potential energy to generate secondary electrons. That means doubly ionized for most metals. Hála et al. ${ }^{136}$ refer to this phase as the high-current metal-dominated phase-intense emission from neutrals and ions of the sputtered vapor dominates the discharge as seen in Fig. 20 (b). Čapek et al. ${ }^{275}$ have recently demonstrated stable sputtering operation $(\Pi=1)$ for a number of target materials and were able to control the current plateau level in the range of 14-105 A by varying the magnetic field strength.

In the case of a decaying discharge current the plasma density decreases due to working gas rarefaction, which reduces the ionization of the working gas as well as sputtered particles and thereby causing a reduced sputtering (i.e., decreasing metal flux). The discharge is here dominated by the neutrals of the working gas and neutral sputtered species 
as in conventional dcMS. The overall effect on the gas dynamics is a decrease of the gas heating (fewer collisions) and ultimately a reduction of the gas depletion to the level expected in dcMS operation. This is consistent with results from Vitelaru et al. ${ }^{247}$ where they find that emission from the metastable working gas atoms again increases during phases 3-4 as seen in Fig. 15. They also point out that gas refill will start to dominate and thus replenish the supply of Ar. On the other hand, in the case that phase 4 is characterized by a sustained high discharge current the depletion of Ar continues as well as the plasma composition being dominated by ions.

Phase 5 (afterglow) is reached as the HiPIMS pulse is switched off, which is characterized by a sharp drop of the discharge current. During the afterglow, Poolcharuansin and Bradley ${ }^{146}$ detect an initial fast decrease of $n_{\mathrm{e}}$ with a time constant of about $30 \mu$ s followed by a much slower decay rate $(3500 \mu \mathrm{s})$. The effective electron temperature $T_{\text {eff }}$ quickly decays reaching values around $0.2 \mathrm{eV}$, which is sustained during several milliseconds. ${ }^{146}$ In addition, the density and temperature of $\mathrm{Ar}^{\mathrm{m}}$ are rapidly decreasing as the plasma species are lost through recombination and diffusion toward the chamber walls. ${ }^{247}$ The decay of the emission from the argon metastables is characterized by an initial fast decrease followed by a more moderate reduction, where

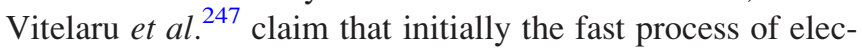
tron impact deexcitation is predominant, while on longer time scales the much slower process of diffusion of $\mathrm{Ar}^{\mathrm{m}}$ prevails. It is worth bearing in mind that the HiPIMS plasma can be sustained during the off-time, where a weak electron density was detected for up to $10 \mathrm{~ms}$ after the pulse was switched off. ${ }^{146}$ Also Hecimovic and Ehiasarian ${ }^{137,175}$ have found that both process gas ions as well as various metal ions are long-lived in the HiPIMS discharge and in some cases present during the entire pulse-off time (up to $10 \mathrm{~ms}$ ), which was described in more detail in Sec. IV B.

\section{SUMMARY}

The HPPMS techniques, of which HiPIMS is the most well-known, are a recent addition to the plasma based sputtering methods. The main feature of HiPIMS is the achievement of a high degree of ionization of the deposition material through the development of a highly dense plasma. This is achieved by using very high pulsed power supplied to the cathode target (magnetron source). The HPPMS techniques have the advantage of essentially using conventional magnetron sputtering setups, except for the power supply. This is a significant advantage over adding a secondary discharge for ionization between the cathode target and the substrate, which is not easily scalable. Thus it also allows utilization of ionized-PVD in industrial systems with rotating targets and configurations with multiple magnetron sources with independent operation. The parameter space for pulsed magnetron sputtering is far from fully explored, since the amplitude, the frequency, as well as the length of the pulse can be modified as long as one stays within the limit of the maximum average power delivered to the magnetron target.
Thus we predict a great and diverse future for HiPIMS, and related techniques, in generating new and better materials and thin film coatings.

In this review the present status of the understanding of the plasma, as generated by HiPIMS, is presented and discussed. It can be concluded that there are still a number of issues regarding the operation of the HiPIMS discharge that are not understood or that are not sufficiently confirmed by the research community. These include the lower deposition rate for HiPIMS compared to dcMS at the same average power, the process gas dynamics, and the transport of charged particles involving classical drift and diffusion as well as anomalous transport. Other important aspects are the high energy tails of the IEDFs, where the acceleration mechanism is not entirely explained, as well as the evolution of the plasma potential, which so far has proven difficult to measure or to control. All these factors and most likely other ones as well need to be mastered in order to progress beyond some of today's rather simplistic understanding of the underlying physical mechanisms. At the same time, computational modeling has the possibility to unify several already existing descriptions into a general description of the coating process, which is more useful for practical purposes. Due to the complexity of the system and the time scales involved, fluid models and PIC simulations may be difficult to implement. Instead a more simple approach has been taken in order to gain understanding of the discharge processes, including the phenomenological material pathway model and the ionization region model. Still much work remains, in particular with making the models generally applicable to different discharge conditions and benchmarking the simulations to experimental results from the HiPIMS community.

\section{ACKNOWLEDGMENTS}

This work was partially supported by Icelandic Research Fund Grant No. 072105003, Swedish Research Council Grant No. 621-2008-3222, and by discussion within the European Collaboration in Science and Technology (COST Action MP0804).

\section{NOMENCLATURE}

$\begin{aligned} A_{\mathrm{t}} & =\text { target area }\left(\mathrm{m}^{2}\right) \\ B & =\text { magnetic field }(\mathrm{T}) \\ C_{\mathrm{s}} & =\text { storage capacitor capacitance }(\mathrm{F}) \\ D_{\mathrm{B}} & =\text { Bohm diffusion coefficient }\left(\mathrm{m}^{2} / \mathrm{s}\right) \\ \mathcal{E}_{\mathrm{b}} & =\text { binding energy of the target material }(\mathrm{eV}) \\ \mathcal{E}_{\mathrm{c}} & =\text { energy loss per electron-ion pair created }(\mathrm{V}) \\ \mathcal{E}_{\mathrm{iz}} & =\text { ionization energy }(\mathrm{eV}) \\ p & =\text { discharge pressure }(\mathrm{Pa}) \\ p_{\mathrm{e}} & =\text { electron pressure }(\mathrm{Pa}) \\ p_{\mathrm{T}} & =\text { peak power density at the target }\left(\mathrm{W} / \mathrm{m}^{2}\right) \\ R & =\text { radius of a cylindrical discharge }(\mathrm{m}) \\ r_{\mathrm{ce}} & =\text { gyration radius of electrons }(\mathrm{m}) \\ r_{\mathrm{ci}} & =\text { gyration radius of ions }(\mathrm{m}) \\ S_{\mathrm{RT}} & =\text { race track area }\left(\mathrm{m}^{2}\right) \\ T_{\mathrm{e}} & =\text { electron temperature }(\mathrm{eV}) \\ T_{\mathrm{g}} & =\text { neutral gas temperature }(\mathrm{K})\end{aligned}$


$\mathbf{u}, u=$ individual particle velocity $(\mathrm{m} / \mathrm{s})$

$u_{\mathrm{e} \perp}=$ electron speed perpendicular to the magnetic field $(\mathrm{m} / \mathrm{s})$

$V_{0}=$ initial peak charge voltage applied to the storage capacitor (V)

$V_{\mathrm{b}}=$ breakdown voltage $(\mathrm{V})$

$V_{\mathrm{d}}=$ discharge voltage or target voltage $(\mathrm{V})$

$V_{\mathrm{IR}}=$ volume of the ionization region $\left(\mathrm{m}^{3}\right)$

$\mathbf{v}, v=$ flow velocity $(\mathrm{m} / \mathrm{s})$

$Y=$ sputter yield

$Y_{\mathrm{c}}=$ compound sputter yield

$Y_{\mathrm{ss}}=$ self-sputter yield

$Y_{\mathrm{t}}=$ effective sputter yield of the target

$Y_{\mathrm{tg}}=$ sputter yield for working gas ion on the target

$\alpha=$ probability of ionization of the sputtered vapor

$\beta_{\mathrm{g}}=$ return probability of ions of the working gas to the target

$\beta_{\mathrm{t}}=$ return probability of ions of the sputtered vapor to the target

$\Gamma=$ flux density of species $\left(\mathrm{m}^{-2} \mathrm{~s}^{-1}\right)$

$\gamma=$ probability a sputtered atom is ionized in the plasma bulk

$\gamma_{\mathrm{SE}}=$ secondary electron emission coefficient

$\gamma_{\mathrm{SE}, \mathrm{eff}}=$ effective secondary electron emission coefficient

$\zeta=$ fraction of the ions impacting the target that are ions of the sputtered vapor

$\eta=$ resistivity $(\Omega \mathrm{m})$

$\eta_{\perp}=$ transverse resistivity or cross $\mathbf{B}$ resistivity $(\Omega \mathrm{m})$

$\theta_{\mathrm{t}}=$ fraction of the race track covered by a compound

$\lambda_{\text {coll }}=$ ion-neutral mean free path $(\mathrm{m})$

$\xi_{\mathrm{i}}=$ probability that an ion of the sputtered vapor ends up at the substrate

$\xi_{\mathrm{n}}=$ probability that a neutral of the sputtered vapor ends up at the substrate

$\Pi=$ generalized self-sputtering parameter

$\Pi_{\mathrm{ss}}=$ self-sputtering parameter

$\sigma=\operatorname{cross}$ section $\left(\mathrm{m}^{2}\right)$

$\sigma_{\mathrm{H}}=$ Hall conductivity $(\mathrm{S} / \mathrm{m})$

$\sigma_{\mathrm{P}}=$ Pedersen conductivity $(\mathrm{S} / \mathrm{m})$

$\tau_{\mathrm{c}}=$ classical collision time $(\mathrm{s})$

$\tau_{\text {eff }}=$ effective collision time (s)

$\Phi=$ electric potential (V)

$\phi=$ work function of the target material $(\mathrm{eV})$

$\psi=$ HiPIMS deposition rate normalized to dcMS deposition rate

$\omega_{\mathrm{e}}=$ electron cyclotron angular frequency $(\mathrm{rad} / \mathrm{s})$

${ }^{1}$ W. D. Gill and E. Kay, Rev. Sci. Instrum. 36, 277 (1965).

${ }^{2}$ J. S. Chapin, Res./Dev. 25, 37 (January 1974).

${ }^{3}$ R. K. Waits, J. Vac. Sci. Technol. 15, 179 (1978).

${ }^{4}$ K. Wasa and S. Hayakawa, Thin Solid Films 52, 31 (1978).

${ }^{5}$ K. Wasa and S. Hayakawa, Thin Solid Films 10, 367 (1972).

${ }^{6}$ J. A. Thornton, J. Vac. Sci. Technol. 11, 666 (1974).

${ }^{7}$ S. M. Rossnagel and J. J. Cuomo, Vacuum 38, 73 (1988).

${ }^{8}$ J. S. Colligon, J. Vac. Sci. Technol. A 13, 1649 (1995).

${ }^{9}$ J. Hopwood, Phys. Plasmas 5, 1624 (1998).

${ }^{10}$ J. A. Hopwood, in Thin Films: Ionized Physical Vapor Deposition, edited by J. A. Hopwood (Academic, San Diego, 2000), pp. 1-7.

${ }^{11}$ U. Helmersson, M. Lattemann, J. Bohlmark, A. P. Ehiasarian, and J. T. Gudmundsson, Thin Solid Films 513, 1 (2006).
${ }^{12}$ S. M. Rossnagel and J. Hopwood, J. Vac. Sci. Technol. B 12, 449 (1994).

${ }^{13}$ S. M. Rossnagel, IBM J. Res. Dev. 43, 163 (1999).

${ }^{14} \mathrm{~S}$. M. Rossnagel, in Handbook of Semiconductor Manufacturing Technology, edited by R. Doering and Y. Nishi, 2nd ed. (CRC Press, Boca Raton, FL, 2008), pp. 15-1-15-27.

${ }^{15}$ J. T. Gudmundsson, J. Phys.: Conf. Ser. 100, 082002 (2008).

${ }^{16}$ V. Kouznetsov, K. Macák, J. M. Schneider, U. Helmersson, and I. Petrov, Surf. Coat. Technol. 122, 290 (1999).

${ }^{17}$ K. Macák, V. Kouznetzov, J. M. Schneider, U. Helmersson, and I. Petrov, J. Vac. Sci. Technol. A 18, 1533 (2000).

${ }^{18}$ A. Anders, Surf. Coat. Technol. 205, S1 (2011).

${ }^{19}$ P. Vašina, M. Meško, J. C. Imbert, M. Ganciu, C. Boisse-Laporte, L. de Poucques, M. Touzeau, D. Pagnon, and J. Bretagne, Plasma Sources Sci. Technol. 16, 501 (2007).

${ }^{20}$ J. Lin, J. J. Moore, W. D. Sproul, B. Mishra, J. A. Rees, Z. Wu, R. Chistyakov, and B. Abraham, Surf. Coat. Technol. 203, 3676 (2009).

${ }^{21}$ J. Sellers, Surf. Coat. Technol. 98, 1245 (1998).

${ }^{22}$ P. J. Kelly, P. S. Henderson, R. D. Arnell, G. A. Roche, and D. Carter, J. Vac. Sci. Technol. A 18, 2890 (2000).

${ }^{23}$ U. Helmersson, M. Lattemann, J. Alami, J. Bohlmark, A. P. Ehiasarian, and J. T. Gudmundsson, in Society of Vacuum Coaters 48th Annual Technical Conference Proceedings, Denver, CO, 23-28, April 2005 (Society of Vacuum Coaters, Albuquerque, NM), pp. 458-464.

${ }^{24}$ K. Sarakinos, J. Alami, and S. Konstantinidis, Surf. Coat. Technol. 204, 1661 (2010).

${ }^{25}$ J. Lin, W. D. Sproul, J. J. Moore, Z. Wu, S. Lee, R. Chistyakov, and B. Abraham,. JOM 63, 48 (2011).

${ }^{26}$ D. Lundin and K. Sarakinos, J. Mater. Res., 27, 780 (2012).

${ }^{27}$ J. Alami, S. Bolz, and K. Sarakinos, J. Alloys Compd. 483, 530 (2009).

${ }^{28}$ E. Kay, F. Parmigiani, and W. Parrish, J. Vac. Sci. Technol. A 6, 3074 (1988).

${ }^{29}$ E. Kusano, in Society of Vacuum Coaters 49th Annual Technical Conference Proceedings, Washington, DC, 22-27 April 2006 (Society of Vacuum Coaters, Albuquerque, NM), pp. 15-20.

${ }^{30}$ J.-W. Lim, H.-S. Park, T.-H. Park, J.-J. Lee, and J. Joo, J. Vac. Sci. Technol. A 18, 524 (2000)

${ }^{31}$ M. Samuelsson, D. Lundin, J. Jensen, M. A. Raadu, J. T. Gudmundsson, and U. Helmersson, Surf. Coat. Technol. 202, 591 (2010).

${ }^{32}$ A. P. Ehiasarian, P. Hovsepian, L. Hultman, and U. Helmersson, Thin Solid Films 457, 270 (2004).

${ }^{33}$ J. Paulitsch, M. Schenkel, T. Zufra $\beta$, P. Mayrhofer, and W.-D. Münz, Thin Solid Films 518, 5558 (2010).

${ }^{34}$ A. P. Ehiasarian, J. G. Wen, and I. Petrov, J. Appl. Phys. 101, 054301 (2007).

${ }^{35}$ K. Sarakinos, J. Alami, and M. Wuttig, J. Phys. D: Appl. Phys. 40, 2108 (2007).

${ }^{36}$ F. Magnus, A. S. Ingason, O. B. Sveinsson, S. Olafsson, and J. T. Gudmundsson, Thin Solid Films 520, 1621 (2011).

${ }^{37}$ J. M. Schneider, W. D. Sproul, A. A. Voevodin, and A. Matthews, J. Vac. Sci. Technol. A 15, 1084 (1997).

${ }^{38}$ J. Alami, P. Eklund, J. M. Andersson, M. Lattemann, E. Wallin, J. Bohlmark, P. Persson, and U. Helmersson, Thin Solid Films 515, 3434 (2007).

${ }^{39}$ J. Bohlmark, M. Östbye, M. Lattemann, H. Ljungcrantz, T. Rosell, and U. Helmersson, Thin Solid Films 515, 1928 (2006).

${ }^{40}$ M. Lattemann, U. Helmersson, and J. E. Greene, Thin Solid Films 518, 5978 (2010).

${ }^{41}$ J. Alami, P. O. A. Petersson, D. Music, J. T. Gudmundsson, J. Bohlmark, and U. Helmersson, J. Vac. Sci. Technol. A 23, 278 (2005).

${ }^{42}$ E. Wallin and U. Helmersson, Thin Solid Films 516, 6398 (2008).

${ }^{43}$ W. R. Grove, Philos. Mag., 4, 498 (1852).

${ }^{44}$ E. Kay, in Advances of Electronics and Electron Physics, edited by L. L. Marton (Academic, New York, 1962), Vol. 17, pp. 245-322.

${ }^{45}$ W. Westwood, Prog. Surf. Sci. 7, 71 (1976).

${ }^{46}$ G. S. Anderson, W. N. Mayer, and G. K. Wehner, J. Appl. Phys. 33, 2991 (1962).

${ }^{47}$ J. L. Vossen and J. J. Cuomo, in Thin Films Processes, edited by J. L. Vossen and W. Kern (Academic, New York, 1978), pp. 11-73.

${ }^{48} \mathrm{~J}$. L. Cecchi, in Handbook of Plasma Processing Technology: Fundamentals, Etching, Deposition, and Surface Engineering, edited by S. M. Rossnagel, J. J. Cuomo, and W. D. Westwood (Noyes, Park Ridge, NJ, 1990), pp. 14-69. 
${ }^{49}$ M. A. Lieberman and A. J. Lichtenberg, Principles of Plasma Discharges and Materials Processing, 2nd ed. (Wiley, New York, 2005).

${ }^{50}$ F. M. Penning, Physica (Amsterdam) 3, 873 (1936).

${ }^{51}$ E. Kay, J. Appl. Phys. 34, 760 (1963).

${ }^{52}$ J. A. Thornton and A. S. Penfold, in Thin Films Processes, edited by J. L. Vossen and W. Kern (Academic, New York, 1978), pp. 76-113.

${ }^{53}$ J. A. Thornton, J. Vac. Sci. Technol. 15, 171 (1978).

${ }^{54}$ K. Wasa and S. Hayakawa, Microelectron. Reliab. 6, 213 (1967).

${ }^{55}$ K. Wasa and S. Hayakawa, Rev. Sci. Instrum. 40, 693 (1969).

${ }^{56}$ B. Window and N. Savvides, J. Vac. Sci. Technol. A 4, 196 (1986).

${ }^{57}$ A. E. Wendt and M. A. Lieberman, J. Vac. Sci. Technol. A 8, 902 (1990).

${ }^{58}$ A. A. Solovev, N. S. Sochugov, K. V. Oskomov, and S. V. Rabotkin, Plasma Phys. Rep. 35, 399 (2009).

${ }^{59}$ A. V. Kozyrev, N. S. Sochugov, K. V. Oskomov, A. N. Zakharov, and A. N. Odivanova, Plasma Phys. Rep. 37, 621 (2011).

${ }^{60}$ S. M. Rossnagel and H. R. Kaufman, J. Vac. Sci. Technol. A 4, 1822 (1986).

${ }^{61}$ T. E. Sheridan, M. J. Goeckner, and J. Goree, J. Vac. Sci. Technol. A 9 , 688 (1991).

${ }^{62}$ S.-H. Seo, J.-H. In, and H.-Y. Chang, Plasma Sources Sci. Technol. 13, 409 (2004).

${ }^{63}$ P. Sigurjonsson and J. T. Gudmundsson, J. Phys.: Conf. Ser. 100, 062018 (2008).

${ }^{64}$ I. Petrov, A. Myers, J. E. Greene, and J. R. Abelson, J. Vac. Sci. Technol. A 12, 2846 (1994).

${ }^{65}$ H. Naghshara, S. Sobhanian, S. Khorram, and N. Sadeghi, J. Phys. D: Appl. Phys. 44, 025202 (2011).

${ }^{66}$ C. Christou and Z. H. Barber, J. Vac. Sci. Technol. A 18, 2897 (2000).

${ }^{67}$ J. T. Gudmundsson, Vacuum 84, 1360 (2010).

${ }^{68}$ S. M. Rossnagel and H. R. Kaufman, J. Vac. Sci. Technol. A 5, 2276 (1987).

${ }^{69}$ S. M. Rossnagel and H. R. Kaufman, J. Vac. Sci. Technol. A 6, 223 (1988).

${ }^{70}$ A. Anders, Surf. Coat. Technol. 183, 301 (2004).

${ }^{71}$ D. Depla, S. Mahieu, and R. De Gryse, Thin Solid Films 517, 2825 (2009).

${ }^{72}$ D. Depla, S. Mahieu, and J. E. Greene, in Handbook of Deposition Technologies for Films and Coatings, 3rd ed., edited by P. M. Martin (William Andrew, Oxford, 2010), Chap. 5, pp. 253-296.

${ }^{73}$ F. F. Chen, Introduction to Plasma Physics and Controlled Fusion (Plenum, New York, 1984), Vol I.

${ }^{74}$ S. M. Rossnagel and H. R. Kaufman, J. Vac. Sci. Technol. A 5, 88 (1987).

${ }^{75}$ W. B. Thompson, An Introduction to Plasma Physics, 2nd ed. (Pergamon, Oxford, 1964) pp. 160-164.

${ }^{76}$ P. J. Kelly and R. D. Arnell, Vacuum 56, 159 (2000).

${ }^{77}$ W. D. Sproul, Vacuum 51, 641 (1998).

${ }^{78}$ P. J. Kelly, R. D. Arnell, W. Ahmed, and A. Afzal, Mater. Des. 17, 215 (1996).

${ }^{79}$ S. J. Nadel, P. Greene, J. Rietzel, and J. Strümpfel, Thin Solid Films 442, 11 (2003).

${ }^{80}$ J. Krempel-Hesse, A. Kloeppel, and M. Hanika, in Proceedings of the 10th International Symposium on Sputtering and Plasma Processes (ISSP 2009), Kanazawa, Japan, July 8-10, 2009 (unpublished), pp. 261-268.

${ }^{81}$ R. S. Nowicki, J. Vac. Sci. Technol. 14, 127 (1977).

${ }^{82}$ S. Schiller, K. Goedicke, J. Reschke, V. Kirchhoff, S. Schneider, and F. Milde, Surf. Coat. Technol. 61, 331 (1993).

${ }^{83}$ R. A. Scholl, Surf. Coat. Technol. 98, 823 (1998).

${ }^{84}$ M. Yamashita, J. Vac. Sci. Technol. A 7, 151 (1989).

${ }^{85}$ S. M. Rossnagel and J. Hopwood, Appl. Phys. Lett. 63, 3285 (1993).

${ }^{86}$ J. Joo, J. Vac. Sci. Technol. A 18, 23 (2000).

${ }^{87}$ S. M. Gorbatkin, D. B. Poker, R. L. Rhoades, C. Doughty, L. A. Berry, and S. M. Rossnagel, J. Vac. Sci. Technol. B 14, 1853 (1996).

${ }^{88}$ J. Musil, S. Kadlec, and W.-D. Münz, J. Vac. Sci. Technol. A 9, 1171 (1991).

${ }^{89}$ Y. Yoshida, Appl. Phys. Lett. 61, 1733 (1992).

${ }^{90}$ J. Xu, X. Deng, J. Zhang, W. Lu, and T. Ma, Thin Solid Films 390, 107 (2001).

${ }^{91}$ J. Hopwood and F. Qian, J. Appl. Phys. 78, 758 (1995).

${ }^{92}$ M. Yamashita, Y. Setsuhara, S. Miyake, M. Kumagai, T. Shoji, and J. Musil, Jpn. J. Appl. Phys., Part 1 38, 4291 (1999).

${ }^{93}$ J. Musil, Vacuum 50, 363 (1998)

${ }^{94}$ J. T. Gudmundsson, J. Alami, and U. Helmersson, Appl. Phys. Lett. 78, 3427 (2001).
${ }^{95}$ J. T. Gudmundsson, J. Alami, and U. Helmersson, Surf. Coat. Technol. 161, 249 (2002).

${ }^{96}$ B. Window and N. Savvides, J. Vac. Sci. Technol. A 4, 453 (1986).

${ }^{97}$ N. Savvides and B. Window, J. Vac. Sci. Technol. A 4, 504 (1986).

${ }^{98}$ T. Iseki, Vacuum 84, 339 (2009).

${ }^{99} \mathrm{~T}$. Iseki, Vacuum 80, 662 (2006).

${ }^{100}$ T. Iseki, Vacuum 84, 1372 (2010).

${ }^{101}$ M. Wright and T. Beardow, J. Vac. Sci. Technol. A 4, 388 (1986).

${ }^{102}$ W. D. Sproul, P. J. Rudnik, M. E. Graham, and S. L. Rohde, Surf. Coat. Technol. 43-44, 270 (1990).

${ }^{103}$ V. Kirchhoff, T. Kopte, T. Winkler, M. Schulze, and P. Wiedemuth, Surf. Coat. Technol. 98, 828 (1998).

${ }^{104}$ M. Åstrand, T. I. Selinder, and M. E. Sjöstrand, Surf. Coat. Technol. 200, 625 (2005).

${ }^{105}$ A. Aijaz, D. Lundin, P. Larsson, and U. Helmersson, Surf. Coat. Technol. 204, 2165 (2010).

${ }^{106}$ V. Stranak, M. Cada, Z. Hubicka, M. Tichy, and R. Hippler, J. Appl. Phys. 108, 043305 (2010).

${ }^{107}$ D. P. Monaghan, D. G. Teer, K. C. Laing, I. Efeoglu, and R. D. Arnell, Surf. Coat. Technol. 59, 21 (1993).

${ }^{108}$ G. N. Glasoe, in Pulse Generators, edited by G. N. Glasoe and J. V. Lebacqz (McGraw-Hill, New York, 1948), pp. 1-17.

${ }^{109}$ J. Markiewicz and J. Emmett, IEEE J. Quantum Electron. 2, 707 (1966).

${ }^{110}$ P. Siemroth, T. Schülke, and T. Witke, Surf. Coat. Technol. 68, 314 (1994).

${ }^{111}$ A. Anders, R. A. MacGill, and T. A. McVeigh, Rev. Sci. Instrum. 70, 4532 (1999).

${ }^{112}$ D. Ochs, P. Ozimek, A. Ehiasarian, and R. Spencer, SVC Bulletin, Spring 2009, pp. 36-39.

${ }^{113}$ D. V. Mozgrin, I. K. Fetisov, and G. V. Khodachenko, Plasma Phys. Rep. 21, 400 (1995).

${ }^{114}$ S. P. Bugaev, N. N. Koval, N. S. Sochugov, and A. N. Zakharov, in $X$ VIIth International Symposium on Discharges and Electrical Insulation in Vacuum, Berkeley, CA 21-26 July 1996 (IEEE, Piscataway, NJ), pp. 1074-1076.

${ }^{115}$ D. J. Christie, F. Tomasel, W. D. Sproul, and D. C. Carter, J. Vac. Sci. Technol. A 22, 1415 (2004).

${ }^{116}$ G. Mark, U.S patent 6,735,099 B2 (17 April 2001).

${ }^{117}$ A. Anders, J. Andersson, and A. Ehiasarian, J. Appl. Phys. 102, 113303 (2007).

${ }^{118}$ J. Musil, J. Leština, J. Vlček, and T. Tölg, J. Vac. Sci. Technol. A 19, 420 (2001).

${ }^{119}$ R. Chistyakov, B. Abraham, and W. D. Sproul, in Society of Vacuum Coaters 49th Annual Technical Conference Proceedings, Washington, DC, 22-27 April 2006 (Society of Vacuum Coaters, Albuquerque, NM), pp. 88-91.

${ }^{120}$ R. Chistyakov, B. Abraham, and W. D. Sproul, J. Moore, and J. Lin, in Society of Vacuum Coaters 50th Annual Technical Conference Proceedings, Louisville, KY, 28 April-3 May 2007 (Society of Vacuum Coaters, Albuquerque, NM), pp. 139-143.

${ }^{121}$ A. P. Ehiasarian and R. Bugyi, in Society of Vacuum Coaters 47 th Annual Technical Conference Proceedings, Dallas, TX, 24-29 April 2004 (Society of Vacuum Coaters, Albuquerque, NM), pp. 486-490.

${ }^{122}$ A. P. Ehiasarian, C. Reinhard, P. E. Hovsepian, and J. M. Colton, in Society of Vacuum Coaters 49th Annual Technical Conference Proceedings, Washington, DC, 22-27 April 2006 (Society of Vacuum Coaters, Albuquerque, NM), pp. 349-353.

${ }_{123}$ A. P. Ehiasarian, Pure Appl. Chem. 82, 1247 (2010).

${ }^{124}$ W. P. Leroy, S. Mahieu, D. Depla, and A. P. Ehiasarian, J. Vac. Sci. Technol. A 28, 108 (2010).

${ }^{125}$ D. Ochs, Vak. Forsch. Prax. 20, 34 (2008).

${ }^{126}$ H. J. White, P. R. Gillette, and J. V. Lebacqz, in Pulse Generators, edited by G. N. Glasoe and J. V. Lebacqz (McGraw-Hill, New York, 1948), pp. $175-224$.

${ }^{127}$ D. J. Christie, W. Sproul, D. Carter, and F. Tomasel, in Society of Vacuum Coaters 47th Annual Technical Conference Proceedings, Dallas, TX, 26-29 April 2004 (Society of Vacuum Coaters, Albuquerque, NM), pp. 113-118.

${ }^{128}$ W. D. Sproul, D. J. Christie, D. C. Carter, F. Tomasel, and T. Linz, Surf. Eng. 20, 174 (2004).

${ }^{129}$ V. Kouznetsov, U.S. patent 6,296,742 B1 (2001).

${ }^{130}$ H. J. White, P. C. Edwards, and H. H. Koski, in Pulse Generators, edited by G. N. Glasoe and J. V. Lebacqz (McGraw-Hill, New York, 1948), pp. $355-416$. 
${ }^{131}$ S. P. Bugaev and N. S. Sochugov, Surf. Coat. Technol. 131, 474 (2000).

${ }^{132} \mathrm{~V}$. Kouznetsov, Method and apparatus for plasma generation, PCT patent WO02103078 (Dec 27, 2002).

${ }^{133}$ I. K. Fetisov, A. A. Filippov, G. V. Khodachenko, D. V. Mozgrin, and A. A. Pisarev, Vacuum 53, 133 (1999).

${ }^{134}$ P. Poolcharuansin, B. Liebig, and J. Bradley, IEEE Trans. Plasma Sci. 38, 3007 (2010).

${ }^{135}$ J. T. Gudmundsson, P. Sigurjonsson, P. Larsson, D. Lundin, and U. Helmersson, J. Appl. Phys. 105, 123302 (2009).

${ }^{136}$ M. Hala, N. Viau, O. Zabeida, J. E. Klemberg-Sapieha, and L. Martinu, J. Appl. Phys. 107, 043305 (2010).

${ }^{137}$ A. Hecimovic and A. P. Ehiasarian, IEEE Trans. Plasma Sci. 39, 1154 (2011).

${ }^{138}$ G. Y. Yushkov and A. Anders, IEEE Trans. Plasma Sci. 38, 3028 (2010).

${ }^{139}$ P. Poolcharuansin and J. Bradley, Surf. Coat. Technol. 205, S307 (2011).

${ }^{140} \mathrm{~J}$. Bohlmark, J. T. Gudmundsson, J. Alami, M. Lattemann, and U. Helmersson, IEEE Trans. Plasma Sci. 33, 346 (2005).

${ }^{141}$ J. Alami, J. T. Gudmundsson, J. Bohlmark, J. Birch, and U. Helmersson, Plasma Sources Sci. Technol. 14, 525 (2005).

${ }^{142}$ A. Vetushka and A. P. Ehiasarian, J. Phys. D: Appl. Phys. 41, 015204 (2008).

${ }^{143}$ A. D. Pajdarová, J. Vlček, P. Kudláček, and J. Lukáš, Plasma Sources Sci. Technol. 18, 025008 (2009).

${ }^{144}$ A. P. Ehiasarian, A. Vetushka, A. Hecimovic, and S. Konstantinidis, J. Appl. Phys. 104, 083305 (2008).

${ }^{145}$ P. Sigurjonsson, P. Larsson, D. Lundin, U. Helmersson, and J. T. Gudmundsson, in Society of Vacuum Coaters 52nd Annual Technical Conference Proceedings, Santa Clara, CA, 9-14 May 2009 (Society of Vacuum Coaters, Albuquerque, NM), pp. 234-239.

${ }^{146}$ P. Poolcharuansin and J. W. Bradley, Plasma Sources Sci. Technol. 19, 025010 (2010).

${ }^{147}$ M. Čada, Z. Hubička, P. Adámek, J. Klusoň, and L. Jastrabík, Surf.Coat. Technol. 205, S317 (2011).

${ }^{148} \mathrm{P}$. Sigurjónsson, "Spatial and temporal variation of the plasma parameters in a high power impulse magnetron sputtering (HiPIMS) discharge," Master's thesis (University of Iceland, Science Institute, 2008), Technical Report RH-14-2008.

${ }^{149}$ K. B. Gylfason, J. Alami, U. Helmersson, and J. T. Gudmundsson, J. Phys. D: Appl. Phys. 38, 3417 (2005).

${ }^{150} \mathrm{~J}$. Böhlmark, "Fundamentals of high power impulse magnetron sputtering," Ph.D. thesis (Linköping University, Sweden, 2006), Linköping Studies in Science and Technology, Dissertation No. 1014.

${ }^{151}$ D. Horwat and A. Anders, J. Appl. Phys. 108, 123306 (2010).

${ }^{152}$ A. P. Ehiasarian, Y. A. Gonzalvo, and T. D. Whitmore, Plasma Process. Polym. 4, S309 (2007).

${ }^{153}$ A. Hecimovic, K. Burcalova, and A. P. Ehiasarian, J. Phys. D: Appl. Phys. 41, 095203 (2008).

${ }^{154}$ L. Meng, A. N. Cloud, S. Jung, and D. N. Ruzic, J. Vac. Sci. Technol. A 29, 011024 (2011).

${ }^{155}$ S.-H. Seo and H.-Y. Chang, J. Appl. Phys. 96, 1310 (2004).

${ }^{156}$ A. E. Ross, R. Sanginés, B. Treverrow, M. M. M. Bilek, and D. R. McKenzie, Plasma Sources Sci. Technol. 20, 035021 (2011).

${ }^{157}$ A. Mishra, P. J. Kelly, and J. W. Bradley, J. Phys. D: Appl. Phys. 44, 425201 (2011).

${ }^{158}$ V. Stranak, S. Drache, M. Cada, Z. Hubicka, M. Tichy, and R. Hippler, Contrib. Plasma Phys. 51, 237 (2011).

${ }^{159}$ O. V. Vozniy, D. Duday, A. Lejars, and T. Wirtz, Vacuum 86, 78 (2011).

${ }^{160}$ I. Petrov, F. Adibi, J. E. Greene, L. Hultman, and J.-E. Sundgren, Appl. Phys. Lett. 63, 36 (1993)

${ }^{161}$ J. M. Andersson, E. Wallin, E. P. Münger, and U. Helmersson, J. Appl. Phys. 100, 033305 (2006).

${ }^{162}$ R. V. Stuart, G. K. Wehner, and G. S. Anderson, J. Appl. Phys. 40, 803 (1969).

${ }^{163}$ M. W. Thompson, Philos. Mag. 18, 377 (1968).

${ }^{164}$ M. W. Thompson, Phys. Rep. 69, 335 (1981).

${ }^{165}$ P. Sigmund, Phys. Rev. 184, 383 (1969).

${ }^{166} \mathrm{H}$. Gnaser, in Sputtering by Particle Bombardment: Experiments and Computer Calculations from Threshold to MeV Energies, edited by R. Behrisch and W. Eckstein (Springer, Berlin, 2007), Chap. 5, pp. 231-328.

${ }^{167}$ J. Bohlmark, M. Lattemann, J. T. Gudmundsson, A. P. Ehiasarian, Y. A. Gonzalvo, N. Brenning, and U. Helmersson, Thin Solid Films 515, 1522 (2006).
${ }^{168}$ D. Lundin, P. Larsson, E. Wallin, M. Lattemann, N. Brenning, and U. Helmersson, Plasma Sources Sci. Technol. 17, 035021 (2008).

${ }^{169}$ P. Poolcharuansin, B. Liebig, and J. W. Bradley, Plasma Sources Sci. Technol. 21, 015001 (2012).

${ }^{170}$ J. Vlček, P. Kudláček, K. Burcalová, and J. Musil, Europhys. Lett. 77, 45002 (2007).

${ }^{171}$ P. Kudláček, J. Vlček, K. Burcalová, and J. Lukáš, Plasma Sources Sci. Technol. 17, 025010 (2008).

${ }^{172}$ J. Lazar, J. Vlček, and J. Rezek, J. Appl. Phys. 108, 063307 (2010).

${ }^{173}$ J. Vlček, A. D. Pajdarova, P. Belsky, J. Lukas, P. Kudlacek, and J. Musil, in Society of Vacuum Coaters 48th Annual Technical Conference Proceedings, Denver, CO, 23-28 April 2005 (Society of Vacuum Coaters, Albuquerque, NM), pp. 465-469.

${ }^{174}$ A. Mishra, G. Clarke, P. Kelly, and J. W. Bradley, Plasma Process. Polym. 6, S610 (2009).

${ }^{175}$ A. Hecimovic and A. P. Ehiasarian, J. Phys. D: Appl. Phys. 42, 135209 (2009).

${ }^{176}$ A. Hecimovic and A. P. Ehiasarian, J. Appl. Phys. 108, 063301 (2010).

${ }^{177}$ J. Bohlmark, J. Alami, C. Christou, A. P. Ehiasarian, and U. Helmersson, J. Vac. Sci. Technol. A 23, 18 (2005).

${ }^{178}$ A. P. Ehiasarian, W.-D. Münz, L. Hultman, U. Helmersson, and I. Petrov, Surf. Coat. Technol. 163-164, 267 (2003).

${ }^{179}$ B. M. DeKoven, P. R. Ward, R. E. Weiss, D. J. Christie, R. A. Scholl, W. D. Sproul, F. Tomasel, and A. Anders, in Society of Vacuum Coaters 46th Annual Technical Conference Proceedings, San Francisco, CA, 3-8 May 2003 (Society of Vacuum Coaters, Albuquerque, NM), pp. $158-165$.

${ }^{180}$ A. Aijaz, K. Sarakinos, D. Lundin, N. Brenning, and U. Helmersson, Diamond and Related Materials 23, 1 (2012).

${ }^{181}$ M. Aiempanakit, U. Helmersson, A. Aijaz, P. Larsson, R. Magnusson, J. Jensen, and T. Kubart, Surf. Coat. Technol. 205, 4828 (2011).

${ }^{182}$ A. P. Ehiasarian, A. Vetushka, Y. A. Gonzalvo, G. Sáfrán, L. Székely, and P. B. Barna, J. Appl. Phys. 109, 104314 (2011).

${ }^{183}$ P.-Y. Jouan, L. Le Brizoual, C. Cardinaud, S. Tricot, and M. A. Djouadi, IEEE Trans. Plasma Sci. 38, 3089 (2010).

${ }^{184}$ G. Greczynski and L. Hultman, Vacuum 84, 1159 (2010).

${ }^{185}$ A. P. Ehiasarian, R. New, W.-D. Münz, L. Hultman, U. Helmersson, and V. Kouznetzov, Vacuum 65, 147 (2002).

${ }^{186}$ J. Andersson, A. P. Ehiasarian, and A. Anders, Appl. Phys. Lett. 93, 071504 (2008).

${ }^{187}$ F. Adibi, I. Petrov, J. E. Greene, L. Hultman, and J. Sundgren, J. Appl. Phys. 73, 8580 (1993).

${ }^{188}$ G. Greczynski, J. Jensen, and L. Hultman, Thin Solid Films 519, 6354 (2011).

${ }^{189}$ D. Lundin, M. Stahl, H. Kersten, and U. Helmersson, J. Phys. D: Appl. Phys. 42, 185202 (2009).

${ }^{190}$ G. West, P. Kelly, P. Barker, A. Mishra, and J. Bradley, Plasma Processes Polym. 6, S543 (2009).

${ }^{191}$ W. P. Leroy, S. Konstantinidis, S. Mahieu, R. Snyders, and D. Depla, J. Phys. D: Appl. Phys. 44, 115201 (2011).

${ }^{192}$ S. Konstantinidis, J. P. Dauchot, M. Ganciu, and M. Hecq, J. Appl. Phys. 99, 013307 (2006).

${ }^{193}$ D. Lundin, N. Brenning, D. Jädernäs, P. Larsson, E. Wallin, M. Lattemann, M. A. Raadu, and U. Helmersson, Plasma Sources Sci. Technol. 18, 045008 (2009).

${ }^{194}$ D. Horwat and A. Anders, J. Phys. D: Appl. Phys. 41, 135210 (2008).

${ }^{195}$ D. J. Christie, J. Vac. Sci. Technol. A 23, 330 (2005).

${ }^{196}$ A. Anders, J. Vac. Sci. Technol. A 28, 783 (2010).

${ }^{197}$ A. Anders, Appl. Phys. Lett. 92, 201501 (2008).

${ }^{198}$ J. Emmerlich, S. Mráz, R. Snyders, K. Jiang, and J. M. Schneider, Vacuum 82, 867 (2008).

${ }^{199}$ J. Alami, K. Sarakinos, G. Mark, and M. Wuttig, Appl. Phys. Lett. 89, 154104 (2006).

${ }^{200}$ A. Mishra, P. J. Kelly, and J. W. Bradley, Plasma Sources Sci. Technol. 19, 045014 (2010).

${ }^{201}$ J. Lin, J. J. Moore, W. D. Sproul, and S. L. Lee, J. Vac. Sci. Technol. A 29, 061301 (2011).

${ }^{202}$ N. Brenning, C. Huo, D. Lundin, M. A. Raadu, C. Vitelaru, G. D. Stancu, T. Minea, and U. Helmersson, "Understanding deposition rate loss in high power impulse magnetron sputtering: I. Ionization driven electric fields," Plasma Sources Sci. Technol. (accepted 2012).

${ }^{203}$ B. Liebig, N. St. J. Braithwaite, P. J. Kelly, and J. W. Bradley, Thin Solid Films 519, 1699 (2010). 
${ }^{204}$ G. Clarke, A. Mishra, P. J. Kelly, and J. W. Bradley, Plasma Process. Polym. 6, S548 (2009).

${ }^{205}$ S. Konstantinidis, J. P. Dauchot, M. Ganciu, and M. Hecq, Appl. Phys. Lett. 88, 021501 (2006).

${ }^{206}$ J. Vlček, B. Zustin, J. Rezek, K. Brucalova, and J. Tesar, in Society of Vacuum Coaters 52nd Annual Technical Conference Proceedings, Santa Clara, CA, 9-14 May 2009 (Society of Vacuum Coaters, Albuquerque, NM), pp. 219-223.

${ }^{207}$ J. Tesar, J. Martan, and J. Rezek, Surf. Coat. Technol. 206, 1155 (2011).

${ }^{208}$ R. A. Baragiola, E. V. Alonso, J. Ferron, and A. Oliva-Florio, Surf. Sci. 90, 240 (1979)

${ }^{209}$ R. A. Baragiola and P. Riccardi, in Reactive Sputter Deposition, edited by D. Depla and S. Mahieu (Springer, Berlin, 2008), pp. 43-60.

${ }^{210} \mathrm{~J}$. Andersson and A. Anders, Appl. Phys. Lett. 92, 221503 (2008).

${ }^{211}$ A. Anders, Surf. Coat. Technol. 204, 2864 (2010).

${ }^{212}$ J. Andersson and A. Anders, Phys. Rev. Lett. 102, 045003 (2009).

${ }^{213}$ D. Horwat and A. Anders, Appl. Phys. Lett. 97, 221501 (2010).

${ }^{214}$ N. Hosokawa, T. Tsukada, and H. Kitahara, in Proceedings of the 8th International Vacuum Congress, Cannes, France, 22-26 September 1980 (unpublished), pp. 11-14.

${ }^{215}$ A. Anders, J. Capek, M. Hála, and L. Martinu, J. Phys. D: Appl. Phys. 45, 012003 (2012).

${ }^{216}$ S. Konstantinidis and R. Snyders, Eur. Phys. J.: Appl. Phys. 56, 24002 (2011).

${ }^{217}$ S. Berg, H.-O. Blom, M. Moradi, C. Nender, and T. Larsson, J. Vac. Sci. Technol. A 7, 1225 (1989).

${ }^{218}$ S. Berg and T. Nyberg, Thin Solid Films 476, 215 (2005).

${ }^{219}$ M. Aiempanakit, T. Kubart, P. Larsson, K. Sarakinos, J. Jensen, and U. Helmersson, Thin Solid Films 519, 7779 (2011).

${ }^{220}$ D. Depla, S. Heirwegh, S. Mahieu, J. Haemers, and R. De Gryse, J. Appl. Phys. 101, 013301 (2007).

${ }^{221}$ J. A. Davis, W. D. Sproul, D. J. Christie, and M. Geisler, in Society of Vacuum Coaters 47th Annual Technical Conference Proceedings, Dallas, TX, 26-29 April 2004 (Society of Vacuum Coaters, Albuquerque, NM), pp. 215-218.

${ }^{222}$ W. Sproul, D. J. Christie, and D. C. Carter, in Society of Vacuum Coaters 47th Annual Technical Conference Proceedings, Dallas, TX, 26-29 April 2004 (Society of Vacuum Coaters, Albuquerque, NM), pp. 96-100.

${ }^{223}$ G. Greczynski, J. Jensen, and L. Hultman, IEEE Trans. Plasma Sci. 38, 3046 (2010).

${ }^{224}$ W. D. Sproul, D. J. Christie, and D. C. Carter, Thin Solid Films 491, 1 (2005).

${ }^{225}$ M. Audronis, V. Bellido-Gonzalez, and B. Daniel, Surf. Coat. Technol. 204, 2159 (2010)

${ }^{226}$ T. Kubart, M. Aiempanakit, J. Andersson, T. Nyberg, S. Berg, and U. Helmersson, Surf. Coat. Technol. 205, S303 (2011).

${ }^{227}$ F. Magnus, O. B. Sveinsson, S. Olafsson, and J. T. Gudmundsson, J. Appl. Phys. 110, 083306 (2011).

${ }^{228}$ C. Costin, L. Marques, G. Popa, and G. Gousset, Plasma Sources Sci. Technol. 14, 168 (2005).

${ }^{229}$ C. Costin, G. Popa, and G. Gousset, J. Optoelectron. Adv. Mater. 7, 2465 (2005).

${ }^{230}$ S. Kondo and K. Nanbu, IEEE Trans. Plasma Sci. 27, 92 (1999).

${ }^{231}$ S. Kondo and K. Nanbu, J. Vac. Sci. Technol. A 19, 830 (2001).

${ }^{232}$ K. Nanbu, K. Mitsui, and S. Kondo, J. Phys. D: Appl. Phys. 33, 2274 (2000).

${ }^{233}$ I. Kolev, A. Bogaerts, and R. Gijbels, Phys. Rev. E 72, 056402 (2005).

${ }^{234}$ I. Kolev and A. Bogaerts, Plasma Process. Polym. 3, 127 (2006).

${ }^{235}$ T. M. Minea, J. Bretagne, and G. Gousset, IEEE Trans. Plasma Sci. 27, 94 (1999).

${ }^{236}$ S. Kondo and K. Nanbu, J. Vac. Sci. Technol. A 19, 838 (2001).

${ }^{237}$ J. Vlček, P. Kudláček, K. Burcalová, and J. Musil, J. Vac. Sci. Technol. A 25, 42 (2007).

${ }^{238}$ J. Vlček and K. Burcalová, Plasma Sources Sci. Technol. 19, 065010 (2010).

${ }^{239}$ D. Lundin, “The HiPIMS process," Ph.D. thesis (Linköping University, Sweden, 2010), Linköping Studies in Science and Technology, Dissertation No. 1305.

${ }^{240}$ H. Matsui, H. Toyoda, and H. Sugai, J. Vac. Sci. Technol. A 23, 671 (2005).
${ }^{241}$ S. M. Rossnagel, J. Vac. Sci. Technol. A 6, 19 (1988).

${ }^{242}$ S. M. Rossnagel, J. Vac. Sci. Technol. A 6, 1821 (1988).

${ }^{243}$ N. Britun, J. G. Han, and S.-G. Oh, Appl. Phys. Lett. 92, 141503 (2008).

${ }^{244}$ D. W. Hoffman, J. Vac. Sci. Technol. A 3, 561 (1985).

${ }^{245}$ J. Olejnicek, H. T. Do, Z. Hubicka, R. Hippler, and L. Jastrabik, Jpn. J. Appl. Phys., Part 1 45, 8090 (2006).

${ }^{246}$ J. Vlček, A. D. Pajdarová, and J. Musil, Contrib. Plasma Phys. 44, 426 (2004).

${ }^{247}$ C. Vitelaru, D. Lundin, G. D. Stancu, N. Brenning, J. Bretagne, and T. Minea, "Argon metastables in HiPIMS.Time-resolved tunable diode laser diagnostic," Plasma Sources Sci. Technol. (accepted 2012).

${ }^{248}$ M. Hála, O. Zabeida, B. Baloukas, J. E. Klemberg-Sapieha, and L. Martinu, IEEE Trans. Plasma Sci. 38, 3035 (2010).

${ }^{249}$ S. Kadlec, Plasma Process. Polym. 4, S419 (2007).

${ }^{250}$ J. W. Coburn and E. Kay, Appl. Phys. Lett. 18, 435 (1971).

${ }^{251}$ E. W. Eckstein, J. W. Coburn, and E. Kay, Int. J. Mass Spectrom. Ion Phys. 17, 129 (1975).

${ }^{252}$ J. A. Hopwood, in Thin Films: Ionized Physical Vapor Deposition, edited by J. A. Hopwood (Academic, San Diego, 2000), pp. 181-207.

${ }^{253}$ S. Ashida, C. Lee, and M. A. Lieberman, J. Vac. Sci. Technol. A 13, 2498 (1995).

${ }^{254}$ J. T. Gudmundsson, J. Phys.: Conf. Ser. 100, 082013 (2008).

${ }^{255}$ M. A. Raadu, I. Axnäs, J. T. Gudmundsson, C. Huo, and N. Brenning, Plasma Sources Sci. Technol. 20, 065007 (2011).

${ }^{256}$ T. Kozák and A. D. Pajdarová, J. Appl. Phys. 110, 103303 (2011).

${ }^{257}$ N. Brenning, I. Axnäs, M. A. Raadu, D. Lundin, and U. Helmerson, Plasma Sources Sci. Technol. 17, 045009 (2008).

${ }^{258}$ C. Huo, M. A. Raadu, D. Lundin, J. T. Gudmundsson, A. Anders, and N. Brenning, "Gas rarefaction and the time evolution of long high power impulse sputtering pulses," Plasma Sources Sci. Technol. (submitted 2011).

${ }^{259}$ O. Msieh, C. Vitelaru, L. De Poucques, D. Saifaoui, and T. Minea, Reactive Sputter Deposition 2010, Ghent, Belgium, 9-10 December 2010 (unpublished).

${ }^{260}$ N. Brenning, R. L. Merlino, D. Lundin, M. A. Raadu, and U. Helmersson, Phys. Rev. Lett. 103, 225003 (2009).

${ }^{261}$ D. Bohm, E. H. S. Burhop, and H. S. W. Massey, in The Characteristics of Electrical Discharges in Magnetic Fields, edited by A. Guthrie and R. K. Wakerling (McGraw-Hill, New York, 1949), Chap. 2, pp. 13-76.

${ }^{262}$ D. Lundin, U. Helmersson, S. Kirkpatrick, S. Rohde, and N. Brenning, Plasma Sources Sci. Technol. 17, 025007 (2008).

${ }^{263}$ J. W. Bradley, S. Thompson, and Y. Aranda-Gonzalvo, Plasma Sources Sci. Technol. 10, 490 (2001).

${ }^{264}$ J. Bohlmark, U. Helmersson, M. VanZeeland, I. Axnäs, J. Alami, and N. Brenning, Plasma Sources Sci. Technol. 13, 654 (2004).

${ }^{265}$ D. Lundin, S. A. Sahab, N. Brenning, C. Huo, and U. Helmersson, Plasma Sources Sci. Technol. 20, 045003 (2011).

${ }^{266}$ T. Hurtig, "Plasma cloud penetration across magnetic boundaries," Ph.D. thesis (Royal Institute of Technology, Stockholm, 2004).

${ }^{267}$ E. Bultinck, S. Mahieu, D. Depla, and A. Bogaerts, J. Phys. D: Appl. Phys. 43, 292001 (2010).

${ }^{268}$ E. Martines, R. Cavazzana, G. Serianni, M. Spolaore, L. Tramontin, M. Zuin, and V. Antoni, Phys. Plasmas 8, 3042 (2001).

${ }^{269}$ E. Martines, M. Zuin, V. Antonia, R. Cavazzana, G. Serianni, M. Spolaore, and C. Nakashima, Phys. Plasmas 11, 1938 (2004).

${ }^{270}$ T. E. Sheridan and J. Goree, J. Vac. Sci. Technol. A 7, 1014 (1989).

${ }^{271}$ B. Angerth, L. Block, U. Fahleson, and K. Soop, Nucl. Fusion 2, 39 (1962).

${ }^{272}$ G. Himmel and A. Piel, J. Phys. D: Appl. Phys. 6, L108 (1973).

${ }^{273}$ A. Piel, E. Möbius, and G. Himmel, Astrophys. Space Sci. 72, 211 (1980).

${ }^{274} \mathrm{~A}$. Anders, P. Ni, and A. Rauch, "Drifting localization of ionization runaway: Unraveling the nature of anomalous transport in high power impulse magnetron sputtering," J. Appl. Phys. (submitted 2011).

${ }^{275}$ J. Čapek, M. Hála, O. Zabeida, J. E. Klemberg-Sapieha, and L. Martinu, J. Appl. Phys. 111, 023301 (2012).

${ }^{276}$ C. Costin, V. Tiron, and G. Popa, IEEE Trans. Plasma Sci. 39, 2482 (2011). 